U.S. DEPARTMENT OF THE INTERIOR U.S. GEOLOGICAL SURVEY

\title{
SEISMICITY MAP OF THE STATE OF IDAHO
}

\author{
By \\ C.W. Stover, B.G. Reagor, and S.T. Algermissen
}

Pamphlet to accompany

MISCELLANEOUS FIELD STUDIES

MAP MF-1857 



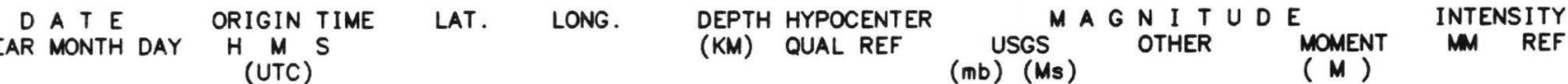
(UTC) (mb) (Ms)

\begin{tabular}{|c|c|c|c|c|c|c|c|c|c|c|c|c|c|c|c|c|c|c|c|}
\hline $\begin{array}{l}1873 \\
1876 \\
1879 \\
1879 \\
1880\end{array}$ & $\begin{array}{l}\text { DEC } \\
\text { APR } \\
\text { NOV } \\
\text { NOV } \\
\text { JUL }\end{array}$ & $\begin{array}{l}18 \\
06 \\
25 \\
26 \\
12\end{array}$ & $\begin{array}{l}14 \\
\cdots \\
\cdots \\
0 \dot{05}\end{array}$ & $\begin{array}{l}\theta 0 \\
\cdots \\
\cdots \\
\ddot{\theta}\end{array}$ & $\begin{array}{l}\cdots \\
\cdots \\
\cdots \\
\cdots\end{array}$ & $\begin{array}{l}42.2 \\
42.0 \\
43.7 \\
43.8 \\
42.0\end{array}$ & $\begin{array}{l}\text { N. } 111.4 \\
\text { N. } 111.3 \\
\text { N. } 116.0 \\
\text { N. } 115.8 \\
\text { N. } 112.2\end{array}$ & $\begin{array}{l}w . \\
w . \\
w . \\
w . \\
w .\end{array}$ & $*$ & $\begin{array}{l}\cdots \\
\cdots \\
\cdots \\
\cdots\end{array}$ & $\begin{array}{l}\mathbf{G} \\
\mathbf{H} \\
\mathbf{G} \\
\mathbf{G} \\
\mathbf{G}\end{array}$ & $\begin{array}{r}298 \\
298 \\
56 \\
56 \\
298\end{array}$ & $\begin{array}{l}\cdots \\
\cdots \\
\cdots \\
\cdots\end{array}$ & $\begin{array}{l}\cdots \\
\cdots \\
\cdots \\
\cdots\end{array}$ & $\begin{array}{l}\cdots \\
\cdots \\
\cdots \\
\cdots \\
\cdots\end{array}$ & $\begin{array}{l}\cdots \\
\cdots \\
\cdots \\
\cdots \\
\cdots\end{array}$ & $\begin{array}{l}\cdots \\
\cdots \\
\cdots \\
\cdots \\
\cdots\end{array}$ & $\begin{array}{l}\cdots \\
\cdots \\
\cdots \\
\cdots\end{array}$ & $\begin{array}{l}\text { IV } \\
\text { I I } \\
\text { I I I * } \\
\text { I I * }\end{array}$ \\
\hline 84 & $\begin{array}{l}\text { JUL } \\
\text { AUG } \\
\text { NOV } \\
\text { Near } \\
\text { North }\end{array}$ & $\begin{array}{l}12 \\
08 \\
10 \\
\text { Pari } \\
\text { hern }\end{array}$ & $\begin{array}{l}05 \\
\dot{08} \\
\text { s, I } \\
\text { Rai I }\end{array}$ & $\begin{array}{l}03 \\
50 \\
\text { I daho } \\
\text { I rood }\end{array}$ & $\begin{array}{l}\cdots \\
\cdots \\
\text { train }\end{array}$ & $\begin{array}{l}42.0 \\
45.5 \\
42.0 \\
\text { mneys } \\
\text { north }\end{array}$ & $\begin{array}{l}\text { N. } 112.0 \\
\text { N. } 115.5 \\
\text { N. } 111.3 \\
\text { were shake } \\
\text { of Ogden, }\end{array}$ & $\begin{array}{l}W . \\
W . \\
W . \\
\text { Ut }\end{array}$ & $\begin{array}{l}\text { * } \\
\text { down } \\
\text { toh. }\end{array}$ & $\begin{array}{l}\cdots \\
\cdots \\
\text { in } \\
\text { Th }\end{array}$ & $\begin{array}{c}G \\
I \\
G \\
P a r\end{array}$ & $\begin{array}{r}298 \\
56 \\
298 \\
\text { is. It } \\
\text { it orea }\end{array}$ & $\begin{array}{l}\cdots \\
\cdots \\
\text { was } \\
\text { wos }\end{array}$ & $\begin{array}{c}\cdots \\
\cdots \\
\text { feit a } \\
\text { estim }\end{array}$ & $\begin{array}{c}\cdots \\
\cdots \\
\text { aboard a } \\
\text { mated at }\end{array}$ & $\begin{array}{l}\cdots \\
\cdots \\
\cdots \\
\text { Utah } \\
\text { t } 15,00\end{array}$ & $\begin{array}{l}\cdots \\
\cdots \\
\text { and } \\
\theta_{\text {sc }}\end{array}$ & $\begin{array}{l}\cdots \\
\cdots \\
\cdots \\
\text { Great } \\
q \mathrm{~km} .\end{array}$ & $\begin{array}{r}F \\
F \\
V I I *\end{array}$ \\
\hline $\begin{array}{l}1884 \\
1884\end{array}$ & $\begin{array}{l}\text { NOV } \\
\text { NOV }\end{array}$ & $\begin{array}{l}10 \\
10\end{array}$ & $\begin{array}{l}09 \\
09\end{array}$ & $\begin{array}{l}10 \\
19\end{array}$ & $\begin{array}{ll}\cdots & 4 \\
\cdots & 4\end{array}$ & $\begin{array}{l}42.0 \\
42.0\end{array}$ & $\begin{array}{l}\text { N. } 111.3 \\
\text { N. } 111.3\end{array}$ & $\begin{array}{l}w . \\
w .\end{array}$ & & & G & $\begin{array}{l}298 \\
298\end{array}$ & $\begin{array}{l}\cdots \\
\cdots\end{array}$ & $\cdots$ & $\begin{array}{l}\cdots \\
\cdots\end{array}$ & $\begin{array}{l}\cdots \\
\cdots\end{array}$ & $\begin{array}{l}\cdots \\
\cdots\end{array}$ & $\cdots$ & $\begin{array}{l}\text { II I } \\
\text { II I }\end{array}$ \\
\hline $\begin{array}{l}1884 \\
1884 \\
1884 \\
1884 \\
1884\end{array}$ & $\begin{array}{l}\text { NOV } \\
\text { NOV } \\
\text { NOV } \\
\text { NOV } \\
\text { NOV }\end{array}$ & $\begin{array}{l}10 \\
10 \\
11 \\
11 \\
12\end{array}$ & $\begin{array}{l}09 \\
10 \\
08 \\
14 \\
08\end{array}$ & $\begin{array}{l}48 \\
45 \\
55 \\
00 \\
50\end{array}$ & $\begin{array}{l}\cdots \\
\cdots \\
\cdots \\
\cdots\end{array}$ & $\begin{array}{l}42.0 \\
42.0 \\
42.0 \\
42.0 \\
42.0\end{array}$ & $\begin{array}{ll}\text { N. } & 111.3 \\
N & 111.3 \\
N . & 111.3 \\
N . & 111.3 \\
N . & 111.3\end{array}$ & $\begin{array}{l}w . \\
w . \\
w . \\
w . \\
w .\end{array}$ & & $\begin{array}{l}\cdots \\
\cdots \\
\cdots \\
\cdots\end{array}$ & $\begin{array}{l}\mathbf{G} \\
\mathbf{G} \\
\mathbf{G} \\
\mathbf{G} \\
\mathbf{G}\end{array}$ & $\begin{array}{l}298 \\
298 \\
298\end{array}$ & $\begin{array}{l}\cdots \\
\cdots \\
\cdots \\
\cdots\end{array}$ & $\begin{array}{l}\cdots \\
\cdots \\
\cdots \\
\cdots\end{array}$ & $\begin{array}{l}\cdots \\
\cdots \\
\cdots \\
\cdots \\
\cdots\end{array}$ & $\begin{array}{l}\cdots \\
\cdots \\
\cdots \\
\cdots \\
\cdots\end{array}$ & $\begin{array}{l}\cdots \\
\cdots \\
\cdots \\
\ldots \\
\cdots\end{array}$ & $\begin{array}{l}\cdots \\
\cdots \\
\cdots \\
\cdots\end{array}$ & $\begin{array}{l}\text { I I } \\
\text { I I } \\
\text { I I } \\
\text { I I } \\
\text { II }\end{array}$ \\
\hline $\begin{array}{l}1884 \\
1884 \\
1884 \\
1884 \\
1893\end{array}$ & $\begin{array}{l}\text { NOV } \\
\text { NOV } \\
\text { NOV } \\
\text { NOV } \\
\text { AUG }\end{array}$ & $\begin{array}{l}12 \\
12 \\
13 \\
13 \\
30\end{array}$ & $\begin{array}{l}09 \\
12 \\
08 \\
10 \\
23\end{array}$ & $\begin{array}{l}35 \\
05 \\
55 \\
40 \\
30\end{array}$ & $\begin{array}{l}\cdots \\
\cdots \\
\cdots \\
\cdots\end{array}$ & $\begin{array}{l}42.0 \\
42.0 \\
42.0 \\
42.0 \\
42.0\end{array}$ & $\begin{array}{ll}\text { N. } & 111.3 \\
\text { N. } & 111.3 \\
N . & 111.3 \\
\text { N. } & 111.3 \\
\text { N. } & 112.7\end{array}$ & $\begin{array}{l}w . \\
w . \\
w . \\
w . \\
w .\end{array}$ & & $\begin{array}{l}\cdots \\
\cdots \\
\cdots \\
\cdots\end{array}$ & $\begin{array}{l}G \\
G \\
G \\
G\end{array}$ & $\begin{array}{l}298 \\
298 \\
298\end{array}$ & $\begin{array}{l}\cdots \\
\cdots \\
\cdots \\
\cdots\end{array}$ & $\begin{array}{l}\cdots \\
\cdots \\
\cdots \\
\cdots\end{array}$ & $\begin{array}{l}\cdots \\
\cdots \\
\cdots \\
\cdots \\
\cdots\end{array}$ & $\begin{array}{l}\cdots \\
\cdots \\
\cdots \\
\cdots \\
\cdots\end{array}$ & $\begin{array}{ll}\cdots & . \\
\cdots & . \\
\cdots & . \\
\cdots & .\end{array}$ & $\begin{array}{l}\cdots \\
\cdots \\
\cdots \\
\cdots\end{array}$ & $\begin{array}{l}\text { I I } \\
\text { II } \\
\text { II } \\
\text { I I } \\
\text { IV }\end{array}$ \\
\hline $\begin{array}{l}1894 \\
1894 \\
i 894 \\
1902 \\
1905\end{array}$ & $\begin{array}{l}\text { DEC } \\
\text { DEC } \\
\text { DEC } \\
\text { JAN } \\
\text { NOV }\end{array}$ & $\begin{array}{l}24 \\
24 \\
05 \\
11\end{array}$ & $\begin{array}{l}12 \\
14 \\
15 \\
01 \\
21\end{array}$ & $\begin{array}{l}\cdots \\
10 \\
14 \\
29\end{array}$ & $\begin{array}{l}\cdots \\
\cdots \\
\cdots \\
\cdots\end{array}$ & $\begin{array}{l}43.6 \\
43.6 \\
43.6 \\
42.2 \\
42.9\end{array}$ & $\begin{array}{ll}\text { N. } & 116.2 \\
\text { N. } & 116.2 \\
\text { N. } & 116.2 \\
\text { N. } & 111.4 \\
\text { N. } & 114.5\end{array}$ & $\begin{array}{l}w . \\
w . \\
w . \\
w . \\
w .\end{array}$ & $\begin{array}{l}* \\
* \\
*\end{array}$ & $\begin{array}{l}\cdots \\
\cdots \\
\cdots \\
\cdots\end{array}$ & $\begin{array}{l}\text { G } \\
\text { G } \\
\text { G } \\
\text { G } \\
\text { H }\end{array}$ & $\begin{array}{r}56 \\
56 \\
56 \\
298 \\
38\end{array}$ & $\begin{array}{l}\cdots \\
\cdots \\
\cdots \\
\cdots\end{array}$ & $\begin{array}{l}\cdots \\
\cdots \\
\cdots \\
\cdots\end{array}$ & $\begin{array}{l}\cdots \\
\cdots \\
\cdots \\
\cdots \\
\cdots\end{array}$ & $\begin{array}{l}\cdots \\
\cdots \\
\cdots \\
\cdots \\
\cdots\end{array}$ & $\begin{array}{ll}\ldots & . \\
\cdots & . \\
\cdots & . \\
\cdots & . \\
\cdots & .\end{array}$ & $\begin{array}{l}\cdots \\
\cdots \\
\cdots \\
\cdots\end{array}$ & $\begin{array}{l}\text { III } \\
\text { II I } \\
V * \\
\text { III } \\
\text { VII }\end{array}$ \\
\hline
\end{tabular}
Neor Shoshone, Idaho. Ploster fell from ceilings in schools and county buildings. County courthouse had crocked wolls. It was felt from Baker. Oregon to Salt Lake City. Utah.

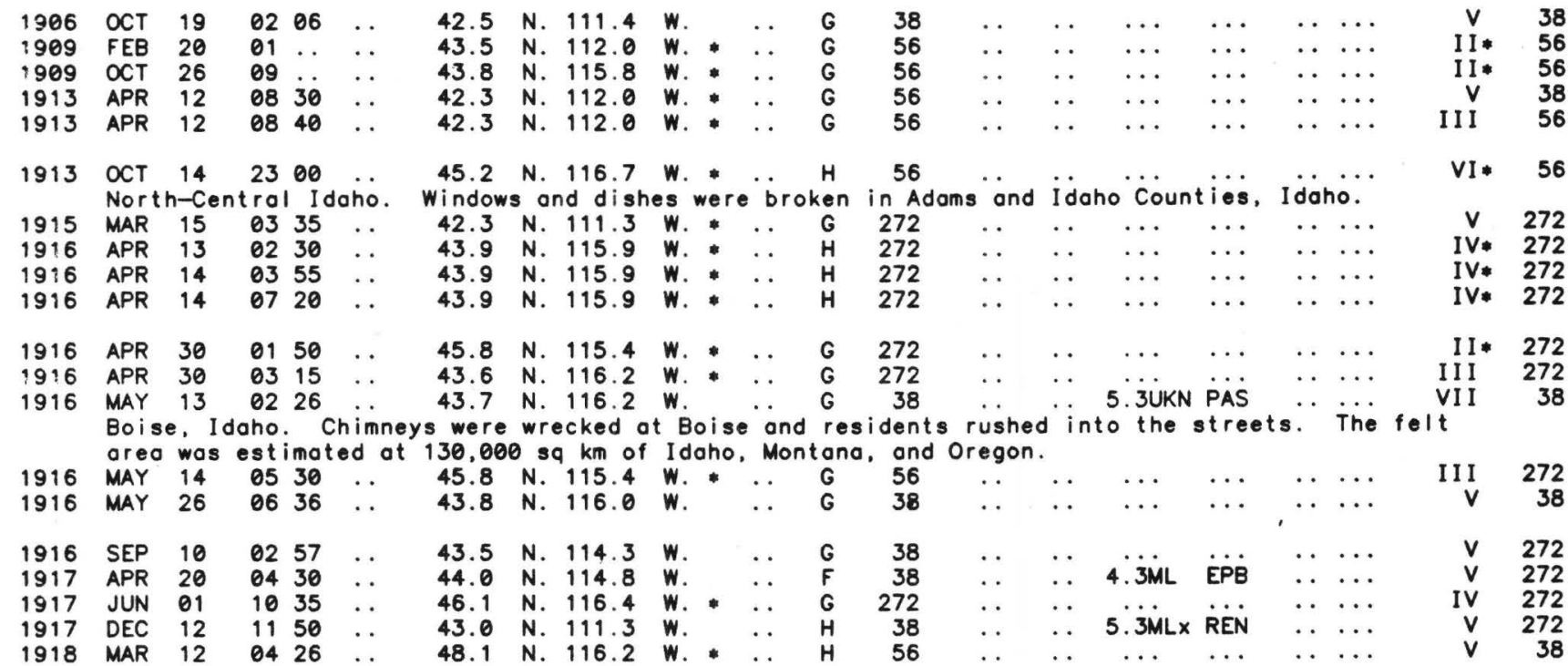




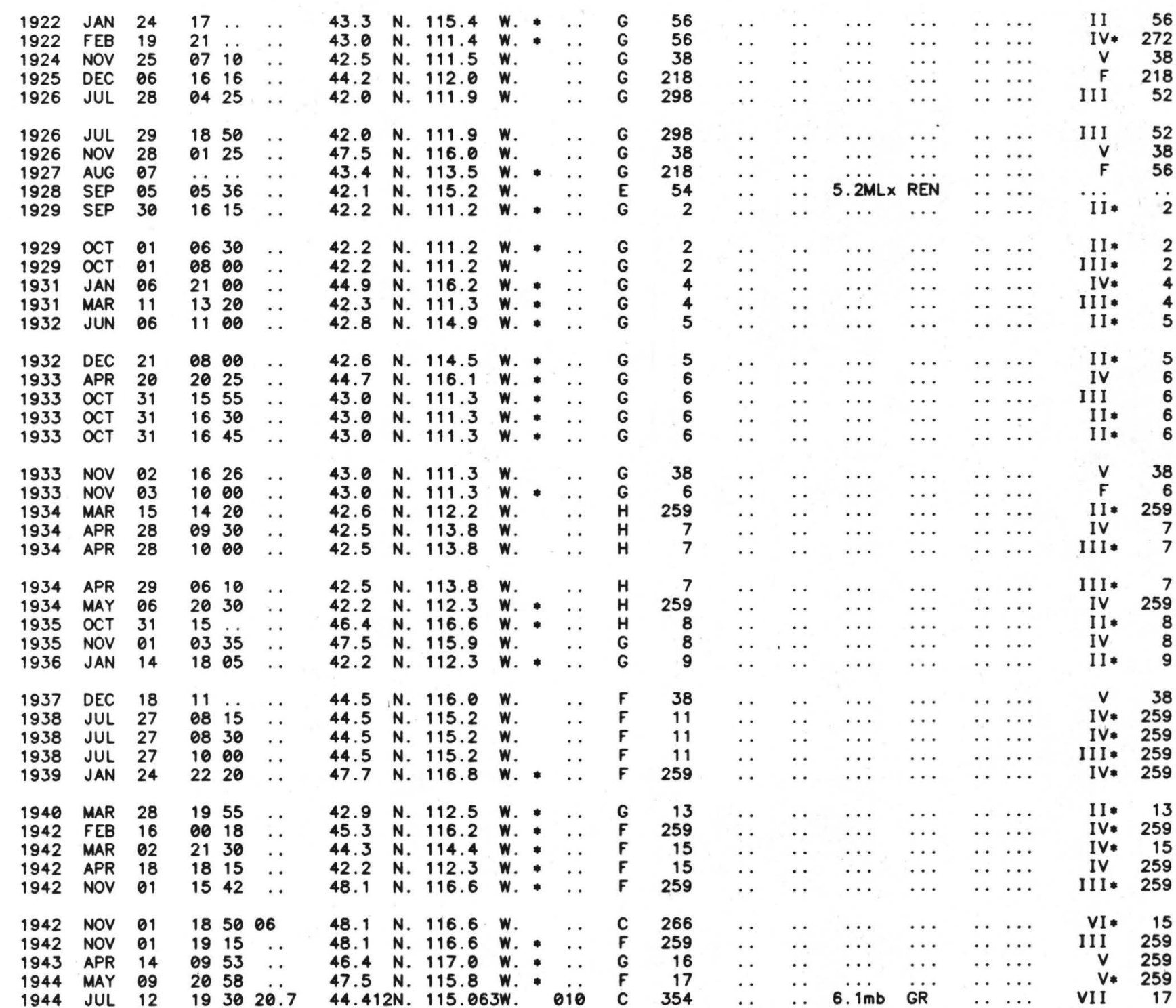
Near Sheep Mountain, Idaho. Domage was most severe in the Fontez Creek area where a new cabin on concrete piers was shaken off its foundation and rock slides occurred on the hillside. A section of the Rapid River Canyon wall near Lime Creedk fell into the river. Sixteen oftershocks were felt. The felt area was estimated at $153,000 \mathrm{sq} \mathrm{km}$ of Idaho, Montano, Oregon, and Washington.

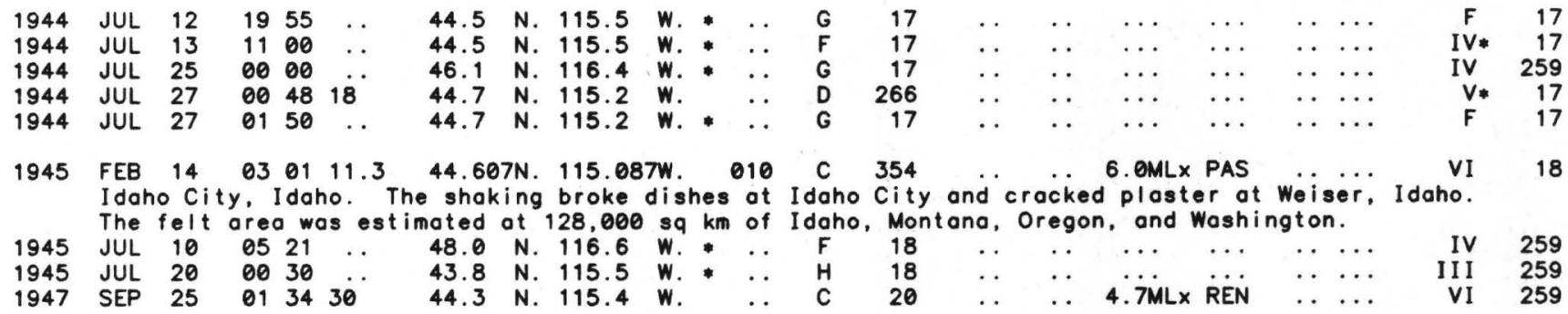


Boise, Idaho. Brick buildings in Boise had several large cracks.
JAN 9906,10

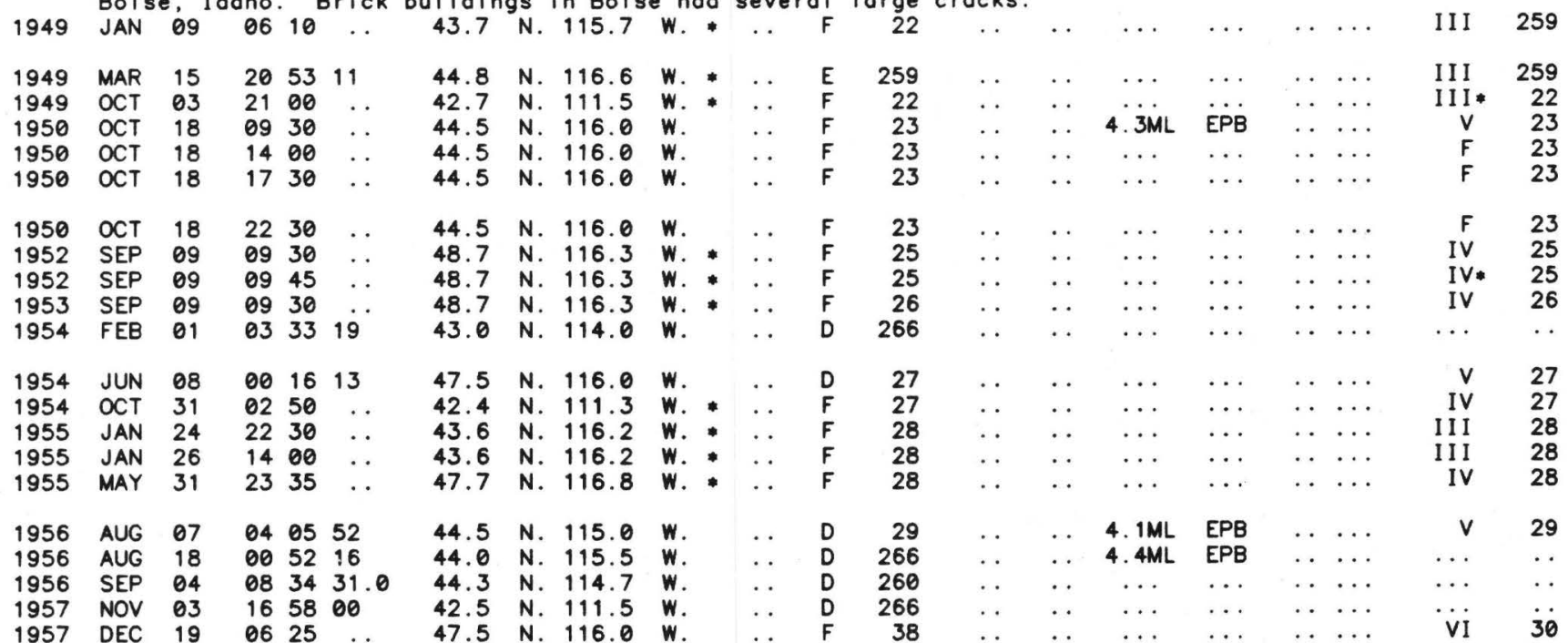

1957 DEC 19 O6 25 Timbers fell and mine walls collapsed at the Galena siliver Mine near Wallace,

Nor I ther

$\begin{array}{llllllllllllll}1958 & \text { MAY } & 23 & 06 & 49 & 47 & 44.5 & \text { N. } & 116.0 & W . & \cdots & D & 31 \\ 1959 & \text { JAN } & 04 & 07 & 22 & \cdots & 42.3 & \text { N. } & 111.4 & W . & * & \cdots & G & 32 \\ 1959 & \text { JAN } & 04 & 12 & 00 & \cdots & 42.3 & \text { N. } 111.4 & W . & * & \cdots & G & 32\end{array}$

1959 SEP $11 \quad 110533$

1960 JAN $03 \quad 0957 \quad 32$

44.5 N. 112.4 W. * . G G

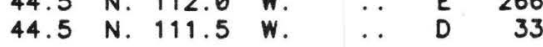

$\begin{array}{lllllllll}31 & \ldots & \ldots & \ldots & \ldots & \ldots & \ldots & \text { IV } & 31\end{array}$

$1960 \quad$ JAN $05 \quad 0403 \quad 34$

1960 APR $24 \quad 203657.0$

1960 MAY O9 234553

1960 JUL $08 \quad 07 \quad 2807$

44.5 N. 111.5 W.

43.0 N. $111.5 \mathrm{~W}$.

44.5 N. 111.5 W

1960 JUL 23

013117.0

42.5 N. 111.5 W.

$\begin{array}{rrr}\text {. } & \text { D } & 33 \\ & D & 266\end{array}$

33
266
33

33
33

$42.5 \mathrm{~N} .111 .5 \mathrm{~W}$.

$\begin{array}{llllll}1960 & \text { JUL } & 23 & 04 & 10 & 13.0 \\ 1960 & \text { JUL } & 23 & 07 & 26 & 49.0\end{array}$

1960 JUL 24 08 05 ..

1960 JUL 24

1960 JUL 25

$0825 \quad$.

42.5 N. 111.5 W.

266

$\cdots \quad \cdots$

$\checkmark \quad 32$

4. $1 \mathrm{ML} \quad \mathrm{EPB}$

iv

1960 JUL $25 \quad 0147 \quad 18.0 \quad 42.5 \quad \mathrm{~N} .111 .5 \quad \mathrm{~W}$

42.5 N. 111.4 W. *..$\quad G \quad G \quad 33$

4.5ML EPB $\ldots . .$. IV 33

1960 AUG $07 \quad 162716.2 \quad 42.4$ N 1115 W. 049 D 266

Necr Soda Springs. Idaho. A ranch located about $15 \mathrm{~km}$ east of Soda Springs hod a cracked

4.5ML EPB $\quad \ldots \quad \ldots \quad \vee \quad \vee \quad 33$

foundation as well as cracked plaster walls. The felt area was estimated at $2,000 \mathrm{sq} \mathrm{km}$.

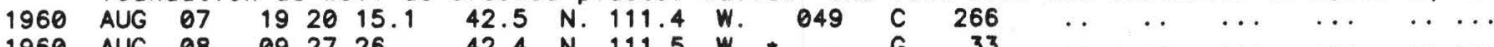

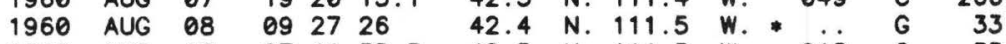

1960 AUG $10 \quad 0741 \quad 35.3 \quad 42.5 \quad \mathrm{~N} .111 .5 \quad \mathrm{~W} .018 \quad 0 \quad 33$

1960 AUG 16

1960 AUG 20

1960 AUG 20

07 $144 \ddot{i}$.

$48.0 \quad$ N. $116.5 \quad$ W. * . . $\quad$ G 33

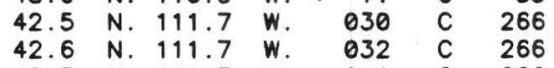

$07 \quad 24 \quad 48.2$

42.3 N. 111.3 W. 041 C 266

III 3 ij

1960 AUG $20 \quad 101140.9$

42.5 N. 111.6 W.

051 C 266

42.6 N. 111.4 W. 014 C 266

42.4 N. 111.5 W. 049 C 266

$\begin{array}{llllll}1960 & \text { AUG } & 20 & 10 & 13 & 14.9 \\ 1960 & \text { AUG } & 20 & 11 & 01 & 59.0\end{array}$

1960 AUG $20 \quad 14 \quad 4651.3$

1960 AUG $21 \quad 050407.3$

42.5 N. 111.6 W.

42.4 N. $111.6 \quad W . \quad 025$ C 266

1960 AUG $26 \quad 01 \quad 22 \quad 39.6$

42.4 N. 111.2 W.

$\begin{array}{lll}025 & C & 266 \\ 032 & C & 266\end{array}$

$\begin{array}{llllll}1960 & \text { OCT } & 15 & 08 & 43 & 11.1\end{array}$

$\begin{array}{llllll}1961 & \text { FEB } & 15 & 09 & 10 & 49.4\end{array}$

42.54 N. $111.83 \mathrm{~W}$

43.4 N. 111.4 W.

005 C 260

1961 APR 11

085752.7

43.4 N. 111.2 W.

$\begin{array}{rrr}033 & C & 266 \\ 033 & C & 74\end{array}$ 
1961 MAY $25 \quad 182803.2 \quad 42.2 \quad$ N. 111.9 W. $033 \quad$ C 34

1961 JUN $11 \quad 1713 \quad 45.5 \quad 44.7$ N. 114.8 W. 025 C 34

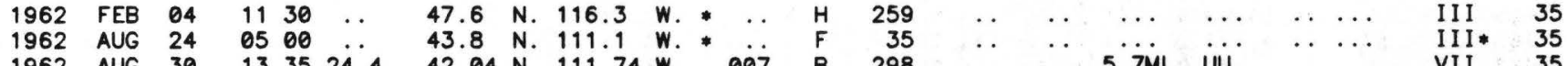

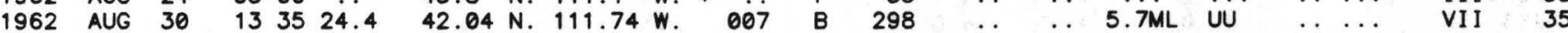

In the Cache Valley near the Idaho-Utah border. Many old brick buildings on the east side of Cache Valley in the orea extending from Logan to Lewiston, Utah had severe damage. Most of the old brick chimneys collapsed and tombstones were overturned. Damage in the area was estimoted ot $\$ 1 \mathrm{milli}$ ion. The felt area was estimated ot $170,000 \mathrm{sq} \mathrm{km}$ of Colorado, Idaho, Nevada, Utah, and Wyoming.

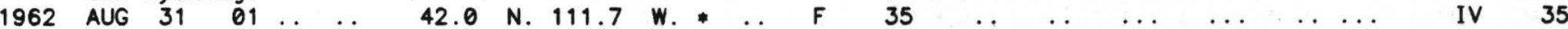

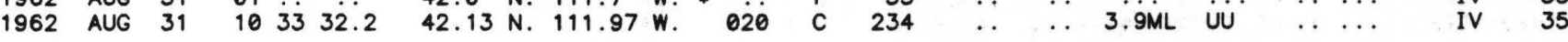

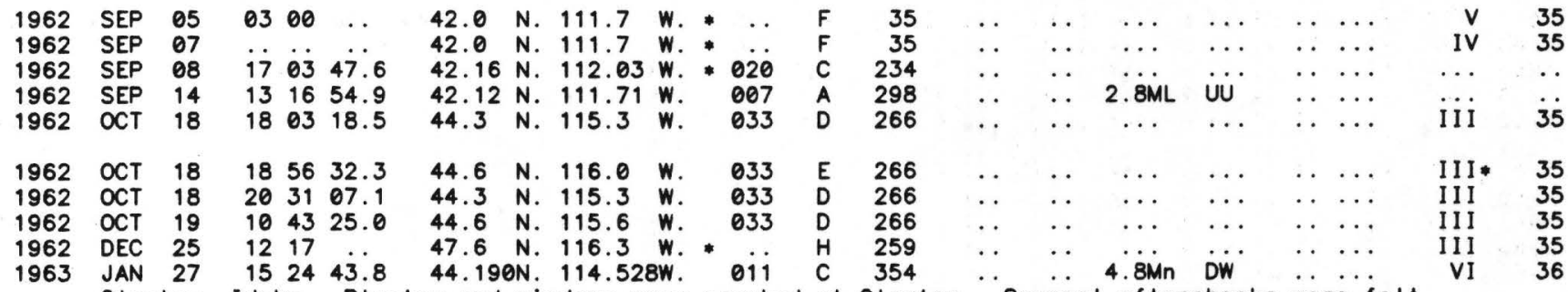
Clayton. Idaho. Plaster and windows were cracked at clayton. Several aftershocks were felt. The felt area was estimated at $15,000 \mathrm{sq} \mathrm{km}$.

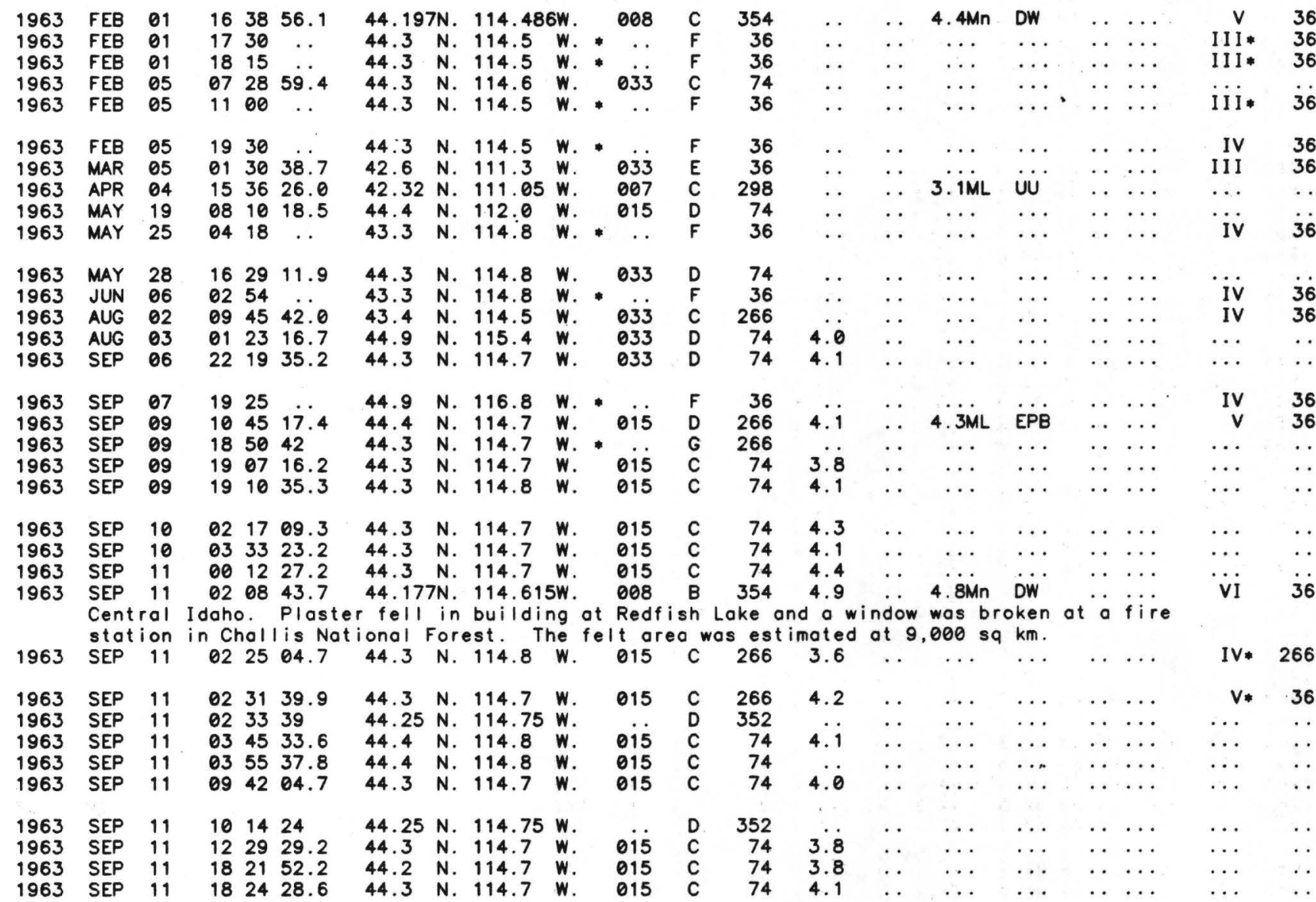




\begin{tabular}{|c|c|c|c|c|c|c|c|c|c|c|c|c|c|c|c|c|c|c|c|c|}
\hline 1963 & SEP & 11 & 20 & 34 & 53.9 & 44.3 & N. & 114.7 & w. & 015 & C & 74 & 3.5 & . & $\cdots$ & $\cdots$ & $\cdots$ & $\cdots$ & $\cdots$ & $\cdots$ \\
\hline $\begin{array}{l}1963 \\
1963 \\
1963 \\
1963 \\
1963\end{array}$ & $\begin{array}{l}\text { SEP } \\
\text { SEP } \\
\text { SEP } \\
\text { SEP } \\
\text { SEP }\end{array}$ & $\begin{array}{l}12 \\
12 \\
12 \\
12 \\
12\end{array}$ & $\begin{array}{l}06 \\
06 \\
08 \\
09 \\
09\end{array}$ & $\begin{array}{l}23 \\
53 \\
01 \\
01 \\
19\end{array}$ & $\begin{array}{l}48.9 \\
00.9 \\
23.2 \\
09.0 \\
04.8\end{array}$ & $\begin{array}{l}44.181 \\
44.2 \\
44.4 \\
44.4 \\
44.3\end{array}$ & $\begin{array}{l}\text { N. } \\
\text { N. } \\
\text { N. } \\
\text { N. } \\
\text { N. }\end{array}$ & $\begin{array}{l}114.62 \\
114.5 \\
114.7 \\
114.8 \\
114.8\end{array}$ & $\begin{array}{l}w . \\
w . \\
w . \\
w . \\
w .\end{array}$ & $\begin{array}{l}009 \\
033 \\
033 \\
015 \\
015\end{array}$ & $\begin{array}{l}B \\
C \\
C \\
C \\
C\end{array}$ & $\begin{array}{r}354 \\
74 \\
266 \\
266 \\
74\end{array}$ & $\begin{array}{l}4.4 \\
4.1 \\
4.3 \\
3.6 \\
3.7\end{array}$ & $\begin{array}{l}\cdots \\
\cdots \\
\cdots \\
\cdots\end{array}$ & $\begin{array}{c}4.7 M n \\
\ldots \\
\ldots \\
\ldots \\
\cdots\end{array}$ & $\begin{array}{l}\text { DW } \\
\ldots \\
\ldots \\
\ldots \\
\cdots\end{array}$ & $\begin{array}{l}\cdots \\
\cdots \\
\cdots \\
\cdots\end{array}$ & $\begin{array}{l}\ldots \\
\ldots \\
\ldots \\
\ldots \\
\cdots\end{array}$ & $\begin{array}{l}\text { IV } \\
\text { IV* } \\
\text { III } \\
\ldots\end{array}$ & $\begin{array}{r}36 \\
266 \\
36 \\
\ldots\end{array}$ \\
\hline $\begin{array}{l}1963 \\
1963 \\
1963 \\
1963 \\
1963\end{array}$ & $\begin{array}{l}\text { SEP } \\
\text { SEP } \\
\text { SEP } \\
\text { SEP } \\
\text { SEP }\end{array}$ & $\begin{array}{l}12 \\
12 \\
12 \\
14 \\
14\end{array}$ & $\begin{array}{l}11 \\
12 \\
20 \\
05 \\
05\end{array}$ & $\begin{array}{l}16 \\
28 \\
15 \\
04 \\
07\end{array}$ & $\begin{array}{l}47.3 \\
22.6 \\
06.3 \\
09.3 \\
14.3\end{array}$ & $\begin{array}{l}44.4 \\
44.3 \\
44.4 \\
44.3 \\
44.4\end{array}$ & $\begin{array}{l}\text { N. } \\
\text { N. } \\
\text { N. } \\
\text { N. } \\
\text { N. }\end{array}$ & $\begin{array}{l}114.7 \\
114.7 \\
114.8 \\
114.8 \\
114.8\end{array}$ & $\begin{array}{l}w . \\
w . \\
w . \\
w . \\
w .\end{array}$ & $\begin{array}{l}015 \\
015 \\
015 \\
015 \\
015\end{array}$ & $\begin{array}{l}C \\
C \\
C \\
C \\
C\end{array}$ & $\begin{array}{l}74 \\
74 \\
74 \\
74 \\
74\end{array}$ & $\begin{array}{l}4.3 \\
4.2 \\
4.0 \\
3.8 \\
3.6\end{array}$ & $\begin{array}{l}\cdots \\
\cdots \\
\cdots \\
\cdots\end{array}$ & $\begin{array}{l}\cdots \\
\cdots \\
\cdots \\
\cdots \\
\cdots\end{array}$ & $\begin{array}{l}\ldots \\
\ldots \\
\ldots \\
\ldots \\
\cdots\end{array}$ & $\begin{array}{l}\cdots \\
\cdots \\
\cdots \\
\cdots\end{array}$ & $\begin{array}{l}\ldots \\
\cdots \\
\cdots \\
\cdots \\
\cdots\end{array}$ & $\begin{array}{l}\ldots \\
\cdots \\
\cdots \\
\cdots \\
\cdots\end{array}$ & $\begin{array}{l}\cdots \\
\cdots \\
\cdots \\
\cdots\end{array}$ \\
\hline $\begin{array}{l}1963 \\
1963 \\
1963 \\
1963 \\
1963\end{array}$ & $\begin{array}{l}\text { SEP } \\
\text { SEP } \\
\text { SEP } \\
\text { SEP } \\
\text { SEP }\end{array}$ & $\begin{array}{l}14 \\
14 \\
14 \\
14 \\
14\end{array}$ & $\begin{array}{l}15 \\
16 \\
16 \\
16 \\
16\end{array}$ & $\begin{array}{l}58 \\
06 \\
25 \\
39 \\
55\end{array}$ & $\begin{array}{l}01.6 \\
49.3 \\
11.3 \\
41.7 \\
40.6\end{array}$ & $\begin{array}{l}44.147 \\
44.3 \\
44.3 \\
44.4 \\
44.3\end{array}$ & $\begin{array}{l}\text { N. } \\
\text { N. } \\
\text { N. } \\
\text { N. } \\
\text { N. }\end{array}$ & $\begin{array}{l}114.625 \\
114.7 \\
114.7 \\
114.7 \\
114.6\end{array}$ & $\begin{array}{l}9 w . \\
w . \\
w . \\
w . \\
w .\end{array}$ & $\begin{array}{l}012 \\
033 \\
015 \\
015 \\
015\end{array}$ & $\begin{array}{l}B \\
C \\
C \\
C \\
C\end{array}$ & $\begin{array}{r}354 \\
74 \\
74 \\
266 \\
266\end{array}$ & $\begin{array}{l}4.3 \\
3.9 \\
4.3 \\
4.0 \\
3.9\end{array}$ & $\begin{array}{l}\cdots \\
\cdots \\
\cdots \\
\cdots\end{array}$ & $\begin{array}{c}4.4 M n \\
\ldots \\
\ldots \\
\ldots \\
\ldots\end{array}$ & $\begin{array}{l}\text { DW } \\
\ldots \\
\cdots \\
\cdots \\
\cdots\end{array}$ & $\begin{array}{l}\cdots \\
\cdots \\
\cdots \\
\cdots\end{array}$ & $\begin{array}{l}\cdots \\
\ldots \\
\cdots \\
\cdots \\
\cdots\end{array}$ & $\begin{array}{c}v \\
\ldots \\
\text { III } \\
\text { III* }\end{array}$ & $\begin{array}{l}36 \\
\ldots \\
36 \\
36\end{array}$ \\
\hline $\begin{array}{l}1963 \\
1963 \\
1963 \\
1963 \\
1963\end{array}$ & $\begin{array}{l}\text { SEP } \\
\text { SEP } \\
\text { SEP } \\
\text { SEP } \\
\text { SEP }\end{array}$ & $\begin{array}{l}14 \\
14 \\
14 \\
15 \\
15\end{array}$ & $\begin{array}{l}17 \\
17 \\
18 \\
05 \\
16\end{array}$ & $\begin{array}{l}16 \\
50 \\
48 \\
34 \\
51\end{array}$ & $\begin{array}{l}34.7 \\
06.9 \\
56.4 \\
57.9 \\
01.8\end{array}$ & $\begin{array}{l}44.3 \\
44.4 \\
44.3 \\
44.3 \\
44.3\end{array}$ & $\begin{array}{l}\text { N. } \\
\text { N. } \\
\text { N. } \\
\text { N. } \\
\text { N. }\end{array}$ & $\begin{array}{l}114.8 \\
114.7 \\
114.8 \\
114.7 \\
114.8\end{array}$ & $\begin{array}{l}w . \\
w . \\
w . \\
w . \\
w .\end{array}$ & $\begin{array}{l}015 \\
015 \\
015 \\
015 \\
015\end{array}$ & $\begin{array}{l}c \\
C \\
C \\
C \\
C\end{array}$ & $\begin{array}{l}74 \\
74 \\
74 \\
74 \\
74\end{array}$ & $\begin{array}{l}3.8 \\
4.0 \\
3.8 \\
3.9 \\
4.1\end{array}$ & $\begin{array}{l}\cdots \\
\cdots \\
\cdots \\
\cdots\end{array}$ & $\begin{array}{l}\ldots \\
\cdots \\
\cdots \\
\cdots \\
\cdots\end{array}$ & $\begin{array}{l}\ldots \\
\cdots \\
\cdots \\
\cdots \\
\cdots\end{array}$ & $\begin{array}{l}\cdots \\
\cdots \\
\cdots \\
\cdots\end{array}$ & $\begin{array}{l}\cdots \\
\cdots \\
\cdots \\
\cdots \\
\cdots\end{array}$ & $\begin{array}{l}\cdots \\
i I I * \\
\ldots \\
\ldots\end{array}$ & $\begin{array}{l}\cdots \\
36 \\
\cdots \\
\cdots\end{array}$ \\
\hline $\begin{array}{l}1963 \\
1963 \\
1963 \\
1963 \\
1963\end{array}$ & $\begin{array}{l}\text { SEP } \\
\text { SEP } \\
\text { SEP } \\
\text { SEP } \\
\text { SEP }\end{array}$ & $\begin{array}{l}15 \\
16 \\
17 \\
17 \\
18\end{array}$ & $\begin{array}{l}19 \\
12 \\
05 \\
12 \\
00\end{array}$ & $\begin{array}{l}14 \\
06 \\
36 \\
22 \\
16\end{array}$ & $\begin{array}{l}09.5 \\
14.0 \\
15.4 \\
54.9 \\
05.9\end{array}$ & $\begin{array}{l}44.2 \\
44.3 \\
44.3 \\
44.4 \\
44.3\end{array}$ & $\begin{array}{l}\text { N. } \\
\text { N. } \\
\text { N. } \\
\text { N. } \\
\text { N. }\end{array}$ & $\begin{array}{l}114.8 \\
114.7 \\
114.8 \\
114.7 \\
114.8\end{array}$ & $\begin{array}{l}w . \\
w . \\
w . \\
w . \\
w .\end{array}$ & $\begin{array}{l}015 \\
015 \\
015 \\
015 \\
015\end{array}$ & $\begin{array}{l}c \\
C \\
C \\
C \\
C\end{array}$ & $\begin{array}{r}74 \\
266 \\
74 \\
74 \\
74\end{array}$ & $\begin{array}{l}3.7 \\
4.2 \\
4.1 \\
3.6 \\
3.7\end{array}$ & $\begin{array}{l}\cdots \\
\cdots \\
\cdots \\
\cdots\end{array}$ & $\begin{array}{l}\cdots \\
\ldots \\
\cdots \\
\ldots \\
\ldots\end{array}$ & $\begin{array}{l}\ldots \\
\cdots \\
\cdots \\
\cdots \\
\cdots\end{array}$ & $\begin{array}{l}\cdots \\
\cdots \\
\cdots \\
\cdots\end{array}$ & $\begin{array}{l}\ldots \\
\ldots \\
\ldots \\
\ldots \\
\ldots\end{array}$ & $\begin{array}{c}\cdots \\
v \\
\cdots \\
\cdots\end{array}$ & $\begin{array}{l}3 \dot{36} \\
\ldots \\
\cdots\end{array}$ \\
\hline $\begin{array}{l}1963 \\
1963 \\
1963 \\
1963 \\
1963\end{array}$ & $\begin{array}{l}\text { SEP } \\
\text { SEP } \\
\text { SEP } \\
\text { SEP } \\
\text { SEP }\end{array}$ & $\begin{array}{l}18 \\
19 \\
19 \\
20 \\
20\end{array}$ & $\begin{array}{l}21 \\
03 \\
10 \\
11 \\
11\end{array}$ & $\begin{array}{l}03 \\
02 \\
59 \\
09 \\
41\end{array}$ & $\begin{array}{l}50.7 \\
05.9 \\
57.0 \\
37.0 \\
20.1\end{array}$ & $\begin{array}{l}44 \cdot 4 \\
44 \cdot 5 \\
44 \cdot 4 \\
44.3 \\
44 \cdot 4\end{array}$ & $\begin{array}{l}\text { N. } \\
\text { N. } \\
\text { N. } \\
\text { N. } \\
\text { N. }\end{array}$ & $\begin{array}{l}114.8 \\
114.7 \\
114.8 \\
114.8 \\
114.7\end{array}$ & $\begin{array}{l}w . \\
w . \\
w . \\
w . \\
w .\end{array}$ & $\begin{array}{l}015 \\
015 \\
015 \\
015 \\
015\end{array}$ & $\begin{array}{l}C \\
C \\
C \\
C \\
C\end{array}$ & $\begin{array}{l}74 \\
74 \\
74 \\
74 \\
74\end{array}$ & $\begin{array}{l}3.8 \\
3.9 \\
3.4 \\
3.8 \\
3.7\end{array}$ & $\begin{array}{l}\cdots \\
\cdots \\
\cdots \\
\cdots\end{array}$ & $\begin{array}{l}\ldots \\
\ldots \\
\ldots \\
\ldots \\
\cdots\end{array}$ & $\begin{array}{l}\cdots \\
\cdots \\
\cdots \\
\cdots \\
\cdots\end{array}$ & $\begin{array}{l}\cdots \\
\cdots \\
\cdots \\
\cdots\end{array}$ & $\begin{array}{l}\ldots \\
\cdots \\
\cdots \\
\cdots \\
\cdots\end{array}$ & $\begin{array}{l}\cdots \\
\cdots \\
\cdots \\
\cdots \\
\cdots\end{array}$ & $\begin{array}{l}\cdots \\
\cdots \\
\cdots \\
\cdots\end{array}$ \\
\hline $\begin{array}{l}1963 \\
1963 \\
1963 \\
1963 \\
1963\end{array}$ & $\begin{array}{l}\text { SEP } \\
\text { SEP } \\
\text { SEP } \\
\text { SEP } \\
\text { SEP }\end{array}$ & $\begin{array}{l}21 \\
21 \\
22 \\
22 \\
22\end{array}$ & $\begin{array}{l}09 \\
12 \\
00 \\
00 \\
04\end{array}$ & $\begin{array}{l}58 \\
29 \\
50 \\
56 \\
37\end{array}$ & $\begin{array}{l}57.5 \\
25.8 \\
36.1 \\
10.5 \\
15.1\end{array}$ & $\begin{array}{l}44.3 \\
43.7 \\
44 \cdot 3 \\
44.3 \\
43 \cdot 3\end{array}$ & $\begin{array}{l}\text { N. } \\
\text { N. } \\
\text { N. } \\
\text { N. } \\
\text { N. }\end{array}$ & $\begin{array}{l}114.8 \\
114.7 \\
114.7 \\
114.8 \\
111.4\end{array}$ & $\begin{array}{l}w . \\
w . \\
w . \\
w . \\
w .\end{array}$ & $\begin{array}{l}015 \\
033 \\
015 \\
015 \\
033\end{array}$ & $\begin{array}{l}C \\
D \\
C \\
C \\
C\end{array}$ & $\begin{array}{r}74 \\
74 \\
74 \\
74 \\
266\end{array}$ & $\begin{array}{c}3.9 \\
3.5 \\
4.2 \\
4.2 \\
\ldots\end{array}$ & $\begin{array}{l}\cdots \\
\cdots \\
\cdots \\
\cdots\end{array}$ & $\begin{array}{l}\ldots \\
\ldots \\
\ldots \\
\ldots \\
\ldots\end{array}$ & $\begin{array}{l}\ldots \\
\cdots \\
\cdots \\
\ldots \\
\cdots\end{array}$ & $\begin{array}{l}\cdots \\
\cdots \\
\cdots \\
\cdots\end{array}$ & $\begin{array}{l}\ldots \\
\ldots \\
\cdots \\
\ldots \\
\ldots\end{array}$ & $\begin{array}{l}\ldots \\
\cdots \\
\cdots \\
\cdots \\
\cdots\end{array}$ & $\begin{array}{l}\cdots \\
\cdots \\
\cdots \\
\cdots\end{array}$ \\
\hline $\begin{array}{l}1963 \\
1963 \\
1963 \\
1963 \\
1963\end{array}$ & $\begin{array}{l}\text { SEP } \\
\text { SEP } \\
\text { SEP } \\
\text { SEP } \\
\text { SEP }\end{array}$ & $\begin{array}{l}22 \\
22 \\
22 \\
22 \\
22\end{array}$ & $\begin{array}{l}06 \\
08 \\
09 \\
14 \\
15\end{array}$ & $\begin{array}{l}30 \\
58 \\
56 \\
55 \\
41\end{array}$ & $\begin{array}{l}01.7 \\
10.5 \\
44.0 \\
01.2 \\
19.0\end{array}$ & $\begin{array}{l}44.3 \\
43 \cdot 3 \\
43 \cdot 3 \\
44 \cdot 4 \\
44 \cdot 4\end{array}$ & $\begin{array}{l}\text { N. } \\
\text { N. } \\
\text { N. } \\
\text { N. } \\
\text { N. }\end{array}$ & $\begin{array}{l}114.8 \\
111.5 \\
111.6 \\
114.8 \\
114.8\end{array}$ & $\begin{array}{l}w . \\
w . \\
w . \\
w . \\
w .\end{array}$ & $\begin{array}{l}015 \\
033 \\
033 \\
015 \\
015\end{array}$ & $\begin{array}{l}c \\
C \\
C \\
C \\
C\end{array}$ & $\begin{array}{l}74 \\
74 \\
74 \\
74 \\
74\end{array}$ & $\begin{array}{r}3.6 \\
3.7 \\
4.1 \\
4.0\end{array}$ & $\begin{array}{l}\cdots \\
\cdots \\
\cdots \\
\cdots\end{array}$ & $\begin{array}{l}\ldots \\
\cdots \\
\cdots \\
\cdots \\
\cdots\end{array}$ & $\begin{array}{l}\ldots \\
\cdots \\
\cdots \\
\cdots \\
\cdots\end{array}$ & $\begin{array}{l}\cdots \\
\cdots \\
\cdots \\
\cdots\end{array}$ & $\begin{array}{l}\cdots \\
\cdots \\
\cdots \\
\cdots \\
\cdots\end{array}$ & $\begin{array}{l}\cdots \\
\cdots \\
\cdots \\
\cdots \\
\cdots\end{array}$ & $\begin{array}{l}\cdots \\
\cdots \\
\cdots \\
\cdots\end{array}$ \\
\hline $\begin{array}{l}1963 \\
1963 \\
1963 \\
1963 \\
1963\end{array}$ & $\begin{array}{l}\text { SEP } \\
\text { SEP } \\
\text { SEP } \\
\text { SEP } \\
\text { SEP }\end{array}$ & $\begin{array}{l}22 \\
22 \\
22 \\
22 \\
23\end{array}$ & $\begin{array}{l}17 \\
21 \\
21 \\
21 \\
01\end{array}$ & $\begin{array}{l}06 \\
13 \\
30 \\
32 \\
30\end{array}$ & $\begin{array}{l}07.1 \\
32.6 \\
55.8 \\
17.0 \\
32.7\end{array}$ & $\begin{array}{l}43.2 \\
44.3 \\
43.2 \\
43.2 \\
43.2\end{array}$ & $\begin{array}{l}\text { N. } \\
\text { N. } \\
\text { N. } \\
\text { N. } \\
\text { N. }\end{array}$ & $\begin{array}{l}111.2 \\
114.8 \\
111.5 \\
111.4 \\
111.3\end{array}$ & $\begin{array}{l}w . \\
w . \\
w . \\
w . \\
w .\end{array}$ & $\begin{array}{l}033 \\
015 \\
033 \\
033 \\
033\end{array}$ & $\begin{array}{l}c \\
C \\
C \\
C \\
C\end{array}$ & $\begin{array}{l}74 \\
74 \\
74 \\
74 \\
74\end{array}$ & $\begin{array}{r}3.9 \\
3.9 \\
3.4 \\
3.5\end{array}$ & $\begin{array}{l}\cdots \\
\cdots \\
\cdots \\
\cdots\end{array}$ & $\begin{array}{l}\ldots \\
\cdots \\
\cdots \\
\cdots \\
\cdots\end{array}$ & $\begin{array}{l}\cdots \\
\cdots \\
\cdots \\
\cdots \\
\cdots\end{array}$ & $\begin{array}{l}\cdots \\
\cdots \\
\cdots \\
\cdots\end{array}$ & $\begin{array}{l}\ldots \\
\cdots \\
\cdots \\
\cdots \\
\cdots\end{array}$ & $\begin{array}{l}\ldots \\
\cdots \\
\cdots \\
\cdots \\
\cdots\end{array}$ & $\begin{array}{l}\cdots \\
\cdots \\
\cdots \\
\cdots\end{array}$ \\
\hline $\begin{array}{l}1963 \\
1963 \\
1963 \\
1963 \\
1963\end{array}$ & $\begin{array}{l}\text { SEP } \\
\text { SEP } \\
\text { SEP } \\
\text { SEP } \\
\text { SEP }\end{array}$ & $\begin{array}{l}23 \\
23 \\
23 \\
24 \\
28\end{array}$ & $\begin{array}{l}10 \\
12 \\
23 \\
17 \\
19\end{array}$ & $\begin{array}{l}21 \\
17 \\
27 \\
05 \\
08\end{array}$ & $\begin{array}{l}03.6 \\
08.6 \\
10.5 \\
27.9 \\
02.7\end{array}$ & $\begin{array}{l}44.4 \\
44.4 \\
43.3 \\
43.2 \\
43.3\end{array}$ & $\begin{array}{l}\text { N. } \\
\text { N. } \\
\text { N. } \\
\text { N. } \\
\text { N. }\end{array}$ & $\begin{array}{l}114.8 \\
114.8 \\
111.5 \\
111.1 \\
111.3\end{array}$ & $\begin{array}{l}w . \\
w . \\
w . \\
w . \\
w .\end{array}$ & $\begin{array}{l}015 \\
015 \\
033 \\
033 \\
033\end{array}$ & $\begin{array}{l}c \\
C \\
C \\
C \\
C\end{array}$ & $\begin{array}{r}74 \\
74 \\
74 \\
266 \\
74\end{array}$ & $\begin{array}{r}3.6 \\
3.9 \\
\ldots \\
3.7\end{array}$ & $\begin{array}{l}\cdots \\
\cdots \\
\cdots \\
\cdots\end{array}$ & $\begin{array}{l}\ldots \\
\cdots \\
\cdots \\
\cdots \\
\cdots\end{array}$ & $\begin{array}{l}\ldots \\
\cdots \\
\cdots \\
\cdots \\
\cdots\end{array}$ & $\begin{array}{l}\cdots \\
\cdots \\
\cdots \\
\cdots\end{array}$ & $\begin{array}{l}\cdots \\
\cdots \\
\cdots \\
\cdots \\
\cdots\end{array}$ & $\begin{array}{l}\ldots \\
\ldots \\
\cdots \\
\cdots \\
\cdots\end{array}$ & $\begin{array}{l}\cdots \\
\cdots \\
\cdots \\
\cdots\end{array}$ \\
\hline $\begin{array}{l}1963 \\
1963 \\
1963 \\
1963 \\
1963\end{array}$ & $\begin{array}{l}\text { SEP } \\
\text { SEP } \\
\text { OCT } \\
\text { OCT } \\
O C T\end{array}$ & $\begin{array}{l}29 \\
29 \\
03 \\
07 \\
11\end{array}$ & $\begin{array}{l}05 \\
06 \\
07 \\
21 \\
23\end{array}$ & $\begin{array}{l}58 \\
05 \\
10 \\
30 \\
09\end{array}$ & $\begin{array}{c}23.7 \\
32.5 \\
30.0 \\
53.1\end{array}$ & $\begin{array}{l}43.4 \\
43.3 \\
44.3 \\
44.8 \\
43.4\end{array}$ & $\begin{array}{l}\text { N. } \\
\text { N. } \\
\text { N. } \\
\text { N. } \\
\text { N. }\end{array}$ & $\begin{array}{l}111.4 \\
111.4 \\
114.4 \\
114.4 \\
111.1\end{array}$ & $\begin{array}{l}w . \\
w . \\
w . \\
w . \\
w .\end{array}$ & $\begin{array}{r}033 \\
033 \\
033 \\
030\end{array}$ & $\begin{array}{l}C \\
C \\
F \\
C \\
C\end{array}$ & $\begin{array}{r}74 \\
74 \\
36 \\
266 \\
266\end{array}$ & $\begin{array}{r}3.6 \\
\ldots \\
3.5 \\
4.3\end{array}$ & $\begin{array}{l}\cdots \\
\cdots \\
\cdots \\
\cdots\end{array}$ & $\begin{array}{l}\ldots \\
\cdots \\
\cdots \\
\cdots \\
\cdots\end{array}$ & $\begin{array}{l}\cdots \\
\cdots \\
\cdots \\
\cdots \\
\cdots\end{array}$ & $\begin{array}{l}\cdots \\
\cdots \\
\cdots \\
\cdots\end{array}$ & $\begin{array}{l}\cdots \\
\cdots \\
\cdots \\
\cdots \\
\cdots\end{array}$ & $\begin{array}{l}\cdots \\
\ddot{\text { IV }} \\
\text { IV } \\
\cdots\end{array}$ & $\begin{array}{l}\cdots \\
36 \\
36 \\
\cdots\end{array}$ \\
\hline $\begin{array}{l}1963 \\
1963\end{array}$ & $\begin{array}{l}\text { OCT } \\
\text { OCT }\end{array}$ & $\begin{array}{l}12 \\
12\end{array}$ & $\begin{array}{l}21 \\
22\end{array}$ & $\begin{array}{l}59 \\
34\end{array}$ & $\begin{array}{l}01.9 \\
01.6\end{array}$ & $\begin{array}{l}43.1 \\
43.1\end{array}$ & $\begin{array}{l}\text { N. } \\
\text { N. }\end{array}$ & $\begin{array}{l}111.1 \\
111.3\end{array}$ & $\begin{array}{l}w . \\
w .\end{array}$ & $\begin{array}{l}033 \\
033\end{array}$ & $\begin{array}{l}C \\
C\end{array}$ & $\begin{array}{l}74 \\
74\end{array}$ & $\begin{array}{l}3.9 \\
3.9\end{array}$ & $\cdots$ & $\cdots$ & $\begin{array}{l}\cdots \\
\cdots\end{array}$ & $\begin{array}{l}\cdots \\
\cdots\end{array}$ & 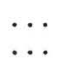 & $\cdots$ & $\begin{array}{l}\cdots \\
\cdots\end{array}$ \\
\hline
\end{tabular}




\begin{tabular}{|c|c|c|c|c|c|c|c|c|c|c|c|c|c|c|c|c|c|c|c|c|}
\hline $\begin{array}{l}1963 \\
1963 \\
1963\end{array}$ & $\begin{array}{l}\text { OCT } \\
\text { OCT } \\
\text { OCT }\end{array}$ & $\begin{array}{l}13 \\
15 \\
16\end{array}$ & $\begin{array}{l}17 \\
15 \\
15\end{array}$ & $\begin{array}{l}55 \\
15 \\
36\end{array}$ & $\begin{array}{l}47.1 \\
10.6 \\
29.1\end{array}$ & $\begin{array}{l}43.2 \\
44.3 \\
44.279\end{array}$ & $\begin{array}{l}\text { N. } \\
\text { N. } \\
9 \mathrm{~N} .\end{array}$ & $\begin{array}{l}111.2 \\
114.8 \\
114.666\end{array}$ & $\begin{array}{l}W . \\
W . \\
6 w .\end{array}$ & $\begin{array}{l}030 \\
033 \\
004\end{array}$ & $\begin{array}{l}\mathrm{C} \\
\mathrm{C} \\
\mathrm{B}\end{array}$ & $\begin{array}{r}74 \\
74 \\
354\end{array}$ & $\begin{array}{l}3.7 \\
3.9 \\
4.2\end{array}$ & $\begin{array}{l}\cdots \\
\cdots \\
\cdots\end{array}$ & 4. & $\ddot{D} \dot{ }$ & $\begin{array}{l}\cdots \\
\cdots \\
\cdots\end{array}$ & $\begin{array}{l}\cdots \\
\cdots \\
\cdots\end{array}$ & iv & $\begin{array}{l}36 \\
\cdots\end{array}$ \\
\hline $\begin{array}{l}1963 \\
1963 \\
1963 \\
1963 \\
1963\end{array}$ & $\begin{array}{l}\text { OCT } \\
\text { OCT } \\
\text { OCT } \\
\text { OCT } \\
\text { OCT }\end{array}$ & $\begin{array}{l}17 \\
24 \\
26 \\
27 \\
29\end{array}$ & $\begin{array}{l}01 \\
09 \\
20 \\
15 \\
05\end{array}$ & $\begin{array}{l}22 \\
52 \\
20 \\
58 \\
39\end{array}$ & $\begin{array}{l}07.7 \\
37.4 \\
14.5 \\
49.9 \\
33.0\end{array}$ & $\begin{array}{l}44.4 \\
44.4 \\
43.1 \\
44.2 \\
43.1\end{array}$ & $\begin{array}{l}\text { N. } \\
\text { N. } \\
\text { N. } \\
\text { N. } \\
\text { N. }\end{array}$ & $\begin{array}{l}114.7 \\
114.8 \\
111.2 \\
114.8 \\
111.6\end{array}$ & $\begin{array}{l}w . \\
w . \\
w . \\
w . \\
w .\end{array}$ & $\begin{array}{l}030 \\
033 \\
037 \\
030 \\
033\end{array}$ & $\begin{array}{l}C \\
C \\
C \\
C \\
C\end{array}$ & $\begin{array}{l}74 \\
74 \\
74 \\
74 \\
74\end{array}$ & $\begin{array}{l}4.7 \\
3.8 \\
4.3 \\
3.6 \\
4.0\end{array}$ & $\begin{array}{l}\cdots \\
\cdots \\
\cdots \\
\cdots\end{array}$ & $\begin{array}{l}\ldots \\
\cdots \\
\cdots \\
\ldots \\
\cdots\end{array}$ & $\begin{array}{l}\cdots \\
\cdots \\
\cdots \\
\cdots \\
\cdots\end{array}$ & $\begin{array}{l}\cdots \\
\cdots \\
\cdots \\
\cdots\end{array}$ & $\begin{array}{l}\cdots \\
\cdots \\
\cdots \\
\cdots \\
\cdots\end{array}$ & $\begin{array}{l}\cdots \\
\cdots \\
\cdots \\
\cdots \\
\cdots\end{array}$ & $\begin{array}{l}\cdots \\
\cdots \\
\cdots \\
\cdots\end{array}$ \\
\hline $\begin{array}{l}1963 \\
1963 \\
1963 \\
1963 \\
1963\end{array}$ & $\begin{array}{l}\text { OCT } \\
\text { OCT } \\
\text { NOV } \\
\text { NOV } \\
\text { NOV }\end{array}$ & $\begin{array}{l}29 \\
31 \\
03 \\
05 \\
28\end{array}$ & $\begin{array}{l}07 \\
08 \\
18 \\
03 \\
03\end{array}$ & $\begin{array}{l}42 \\
08 \\
26 \\
44 \\
14\end{array}$ & $\begin{array}{l}11.8 \\
52.0 \\
02.0 \\
39.7 \\
02.4\end{array}$ & $\begin{array}{l}43.2 \\
43.0 \\
43.1 \\
43.1 \\
44.3\end{array}$ & $\begin{array}{l}\text { N. } \\
\text { N. } \\
\text { N. } \\
\text { N. } \\
\text { N. }\end{array}$ & $\begin{array}{l}111.3 \\
111.3 \\
111.3 \\
111.2 \\
114.8\end{array}$ & $\begin{array}{l}W . \\
w . \\
w . \\
W . \\
w .\end{array}$ & $\begin{array}{l}030 \\
033 \\
033 \\
033 \\
033\end{array}$ & $\begin{array}{l}c \\
C \\
C \\
C \\
C\end{array}$ & $\begin{array}{l}74 \\
74 \\
74 \\
74 \\
74\end{array}$ & $\begin{array}{l}3.0 \\
4.2 \\
3.9 \\
3.5\end{array}$ & $\begin{array}{l}\cdots \\
\cdots \\
\cdots \\
\cdots\end{array}$ & $\begin{array}{l}\ldots \\
\ldots \\
\ldots \\
\ldots \\
\cdots\end{array}$ & $\begin{array}{l}\cdots \\
\cdots \\
\cdots \\
\cdots \\
\cdots\end{array}$ & $\begin{array}{l}\cdots \\
\cdots \\
\cdots \\
\cdots\end{array}$ & $\begin{array}{l}\ldots \\
\cdots \\
\cdots \\
\cdots \\
\cdots\end{array}$ & $\begin{array}{l}\cdots \\
\cdots \\
\cdots \\
\cdots \\
\cdots\end{array}$ & $\begin{array}{l}\cdots \\
\cdots \\
\cdots \\
\cdots\end{array}$ \\
\hline $\begin{array}{l}1963 \\
1963 \\
1963 \\
1963 \\
1963\end{array}$ & $\begin{array}{l}\text { DEC } \\
\text { DEC } \\
\text { DEC } \\
\text { DEC } \\
\text { DEC }\end{array}$ & $\begin{array}{l}14 \\
22 \\
22 \\
23 \\
23\end{array}$ & $\begin{array}{l}18 \\
02 \\
05 \\
00 \\
00\end{array}$ & $\begin{array}{l}46 \\
50 \\
44 \\
15 \\
28\end{array}$ & $\begin{array}{l}37.7 \\
29.8 \\
39.2 \\
02.0 \\
59.1\end{array}$ & $\begin{array}{l}44.5 \\
44.4 \\
44.4 \\
44.3 \\
44.2\end{array}$ & $\begin{array}{l}\text { N. } \\
\text { N. } \\
\text { N. } \\
\text { N. } \\
\text { N. }\end{array}$ & $\begin{array}{l}114.8 \\
114.6 \\
114.5 \\
114.7 \\
114.4\end{array}$ & $\begin{array}{l}w . \\
w . \\
w . \\
w . \\
w .\end{array}$ & $\begin{array}{l}033 \\
033 \\
033 \\
033 \\
033\end{array}$ & $\begin{array}{l}C \\
C \\
C \\
C \\
C\end{array}$ & $\begin{array}{r}266 \\
74 \\
74 \\
266 \\
74\end{array}$ & $\begin{array}{r}3.9 \\
4.4 \\
4.1 \\
3.8\end{array}$ & $\begin{array}{l}\cdots \\
\cdots \\
\cdots \\
\cdots\end{array}$ & $\begin{array}{c}\cdots \\
\ldots \\
5.1 M L \\
\ldots\end{array}$ & $\begin{array}{c}\cdots \\
\cdots \\
\dot{E} P B \\
\cdots\end{array}$ & $\begin{array}{l}\cdots \\
\cdots \\
\cdots \\
\cdots\end{array}$ & $\begin{array}{l}\cdots \\
\cdots \\
\cdots \\
\cdots \\
\cdots\end{array}$ & $\begin{array}{l}\cdots \\
\cdots \\
\cdots \\
\cdots \\
\cdots\end{array}$ & $\begin{array}{l}\cdots \\
\cdots \\
\cdots \\
\cdots\end{array}$ \\
\hline $\begin{array}{l}1963 \\
1964 \\
1964 \\
1964 \\
1964\end{array}$ & $\begin{array}{l}\text { DEC } \\
\text { JAN } \\
\text { JAN } \\
\text { JAN } \\
\text { JAN }\end{array}$ & $\begin{array}{l}25 \\
06 \\
09 \\
09 \\
10\end{array}$ & $\begin{array}{l}20 \\
19 \\
03 \\
11 \\
08\end{array}$ & $\begin{array}{l}04 \\
35 \\
10 \\
11 \\
52\end{array}$ & $\begin{array}{l}10.2 \\
07.9 \\
56.4 \\
54.3 \\
18.0\end{array}$ & $\begin{array}{l}44.2 \\
44.272 \\
44.257 \\
44.3 \\
43.9\end{array}$ & $\begin{array}{l}\text { N. } \\
2 N . \\
\text { NN. } \\
\text { N. } \\
\text { N. }\end{array}$ & $\begin{array}{l}114.5 \\
114.443 \\
114.472 \\
114.8 \\
111: 4\end{array}$ & $\begin{array}{l}w . \\
3 w . \\
2 W . \\
w . \\
w .\end{array}$ & $\begin{array}{l}033 \\
010 \\
024 \\
015 \\
033\end{array}$ & $\begin{array}{l}C \\
B \\
B \\
C \\
D\end{array}$ & $\begin{array}{r}74 \\
354 \\
354 \\
266 \\
299\end{array}$ & $\begin{array}{c}3.7 \\
4.7 \\
4.5 \\
3.6 \\
\ldots\end{array}$ & $\begin{array}{l}\cdots \\
\cdots \\
\cdots \\
\cdots\end{array}$ & $\begin{array}{c}\ldots \\
4.2 \mathrm{Mn} \\
4.0 \mathrm{Mn} \\
\ldots \\
\ldots\end{array}$ & $\begin{array}{l}\cdots \\
\text { DW } \\
\cdots \\
\cdots\end{array}$ & $\begin{array}{l}\cdots \\
\cdots \\
\cdots \\
\cdots\end{array}$ & $\begin{array}{l}\cdots \\
\cdots \\
\cdots \\
\cdots \\
\cdots\end{array}$ & $\begin{array}{l}\cdots \\
\cdots \\
\cdots \\
\cdots \\
\cdots\end{array}$ & $\begin{array}{l}\cdots \\
\cdots \\
\cdots \\
\cdots\end{array}$ \\
\hline $\begin{array}{l}1964 \\
1964 \\
1964 \\
1964 \\
1964\end{array}$ & $\begin{array}{l}\text { JAN } \\
\text { JAN } \\
\text { JAN } \\
\text { JAN } \\
\text { JAN }\end{array}$ & $\begin{array}{l}20 \\
22 \\
23 \\
28 \\
30\end{array}$ & $\begin{array}{l}10 \\
21 \\
03 \\
12 \\
22\end{array}$ & $\begin{array}{l}09 \\
10 \\
04 \\
57 \\
23\end{array}$ & $\begin{array}{l}39.7 \\
58.8 \\
49.7 \\
07.9 \\
10.4\end{array}$ & $\begin{array}{l}43.34 \\
44.3 \\
44.4 \\
43.2 \\
43.3\end{array}$ & $\begin{array}{l}\text { N. } \\
\text { N. } \\
\text { N. } \\
\text { N. } \\
\text { N. }\end{array}$ & $\begin{array}{l}112.45 \\
114.8 \\
114.5 \\
111.4 \\
111.4\end{array}$ & $\begin{array}{l}w . \\
w . \\
w . \\
w . \\
w .\end{array}$ & $\begin{array}{l}005 \\
033 \\
033 \\
041 \\
033\end{array}$ & $\begin{array}{l}c \\
C \\
C \\
C \\
C\end{array}$ & $\begin{array}{l}260 \\
266 \\
266 \\
266 \\
266\end{array}$ & $\begin{array}{c}3.9 \\
4.1 \\
4.2 \\
\ldots\end{array}$ & $\begin{array}{l}\cdots \\
\cdots \\
\cdots \\
\cdots\end{array}$ & $\begin{array}{l}\ldots \\
\ldots \\
\ldots \\
\ldots \\
\cdots\end{array}$ & $\begin{array}{l}\ldots \\
\cdots \\
\ldots \\
\cdots \\
\cdots\end{array}$ & $\begin{array}{l}\cdots \\
\cdots \\
\cdots \\
\cdots\end{array}$ & $\begin{array}{l}\ldots \\
\cdots \\
\cdots \\
\cdots \\
\cdots\end{array}$ & $\begin{array}{l}\cdots \\
\cdots \\
\cdots \\
\cdots \\
\cdots\end{array}$ & $\begin{array}{l}\cdots \\
\cdots \\
\cdots \\
\cdots\end{array}$ \\
\hline $\begin{array}{l}1964 \\
1964 \\
1964 \\
1964 \\
1964\end{array}$ & $\begin{array}{l}\text { FEB } \\
\text { FEB } \\
\text { FEB } \\
\text { FEB } \\
\text { FEB }\end{array}$ & $\begin{array}{l}02 \\
03 \\
06 \\
06 \\
07\end{array}$ & $\begin{array}{l}12 \\
05 \\
08 \\
11 \\
13\end{array}$ & $\begin{array}{l}15 \\
55 \\
02 \\
13 \\
20\end{array}$ & $\begin{array}{l}11.0 \\
44.3 \\
26.4 \\
33.2 \\
06.6\end{array}$ & $\begin{array}{l}43.3 \\
43.2 \\
42.06 \\
42.08 \\
42.10\end{array}$ & $\begin{array}{l}\text { N. } \\
\text { N. } \\
\text { N. } \\
\text { N. } \\
\text { N. }\end{array}$ & $\begin{array}{l}111.4 \\
111.1 \\
112.20 \\
112.33 \\
112.29\end{array}$ & $\begin{array}{l}w . \\
w . \\
w . \\
w . \\
w .\end{array}$ & $\begin{array}{l}030 \\
030 \\
007 \\
007 \\
007\end{array}$ & $\begin{array}{l}C \\
C \\
B \\
B \\
B\end{array}$ & $\begin{array}{l}266 \\
266 \\
298 \\
298 \\
298\end{array}$ & $\begin{array}{c}\ddot{j} \\
\ldots \\
\cdots \\
\cdots\end{array}$ & $\begin{array}{l}\cdots \\
\cdots \\
\cdots \\
\cdots\end{array}$ & $\begin{array}{c}\ldots \\
2 . \ddot{4 M L} \\
2.6 \mathrm{ML} \\
\ldots\end{array}$ & $\begin{array}{l}\cdots \\
\ddot{U U U} \\
\cdots\end{array}$ & $\begin{array}{l}\cdots \\
\cdots \\
\cdots \\
\cdots\end{array}$ & $\begin{array}{l}\cdots \\
\cdots \\
\cdots \\
\cdots \\
\cdots\end{array}$ & $\begin{array}{l}\cdots \\
\text { III } \\
\text { III } \\
\ldots\end{array}$ & $\begin{array}{l}\cdots \\
37 \\
37 \\
\ldots\end{array}$ \\
\hline $\begin{array}{l}1964 \\
1964 \\
1964 \\
1964 \\
1964\end{array}$ & $\begin{array}{l}\text { FEB } \\
\text { FEB } \\
\text { MAR } \\
\text { APR } \\
\text { APR }\end{array}$ & $\begin{array}{l}08 \\
20 \\
28 \\
12 \\
13\end{array}$ & $\begin{array}{l}06 \\
03 \\
13 \\
15 \\
11\end{array}$ & $\begin{array}{l}22 \\
29 \\
00 \\
37 \\
36\end{array}$ & $\begin{array}{l}07.5 \\
36.1 \\
24 \\
49.6 \\
30.1\end{array}$ & $\begin{array}{l}44.206 \\
44.4 \\
44.5 \\
43.2 \\
43.34\end{array}$ & $\begin{array}{l}6 \mathrm{~N} . \\
\text { N. } \\
\text { N. } \\
\text { N. } \\
\text { N. }\end{array}$ & $\begin{array}{l}114.477 \\
114.7 \\
112.3 \\
111.4 \\
111.10\end{array}$ & $\begin{array}{l}7 w . \\
w . \\
w . \\
w . \\
w .\end{array}$ & $\begin{array}{l}017 \\
030 \\
033 \\
015 \\
015\end{array}$ & $\begin{array}{l}B \\
D \\
D \\
C \\
D\end{array}$ & $\begin{array}{r}354 \\
266 \\
299 \\
74 \\
299\end{array}$ & $\begin{array}{c}4.3 \\
3.7 \\
\ldots \\
\ldots \\
\ldots\end{array}$ & $\begin{array}{l}\cdots \\
\cdots \\
\cdots \\
\cdots\end{array}$ & $\begin{array}{c}4.1 M n \\
\ldots \\
\ldots \\
\ldots \\
\ldots\end{array}$ & $\begin{array}{l}\text { DW } \\
\ldots \\
\ldots \\
\ldots \\
\cdots\end{array}$ & $\begin{array}{l}\cdots \\
\cdots \\
\cdots \\
\cdots\end{array}$ & $\begin{array}{l}\cdots \\
\cdots \\
\cdots \\
\cdots \\
\cdots\end{array}$ & $\begin{array}{l}\cdots \\
\cdots \\
\cdots \\
\cdots \\
\cdots\end{array}$ & $\begin{array}{l}\cdots \\
\cdots \\
\cdots \\
\cdots\end{array}$ \\
\hline $\begin{array}{l}1964 \\
1964 \\
1964 \\
1964 \\
1964\end{array}$ & $\begin{array}{l}\text { APR } \\
\text { APR } \\
\text { APR } \\
\text { APR } \\
\text { MAY }\end{array}$ & $\begin{array}{l}15 \\
17 \\
21 \\
21 \\
08\end{array}$ & $\begin{array}{l}13 \\
06 \\
09 \\
12 \\
01\end{array}$ & $\begin{array}{l}37 \\
53 \\
48 \\
11 \\
35\end{array}$ & $\begin{array}{l}02.7 \\
43.6 \\
42 \\
32.9 \\
08.2\end{array}$ & $\begin{array}{l}43.1 \\
44.1 \\
44.7 \\
44.2 \\
43.3\end{array}$ & $\begin{array}{l}\text { N. } \\
N . \\
N . \\
N . \\
N .\end{array}$ & $\begin{array}{l}111.5 \\
114.3 \\
115.1 \\
114.3 \\
111.3\end{array}$ & $\begin{array}{l}w . \\
w . \\
w . \\
w . \\
w .\end{array}$ & $\begin{array}{l}033 \\
033 \\
033 \\
033 \\
033\end{array}$ & $\begin{array}{l}D \\
D \\
D \\
D \\
D\end{array}$ & $\begin{array}{l}266 \\
266 \\
299 \\
266 \\
266\end{array}$ & $\begin{array}{c}3 . \dot{6} \\
3 . \dot{6} \\
\ldots\end{array}$ & $\begin{array}{l}\cdots \\
\cdots \\
\cdots \\
\cdots\end{array}$ & $\begin{array}{l}\ldots \\
\ldots \\
\cdots \\
\cdots\end{array}$ & $\begin{array}{l}\cdots \\
\cdots \\
\cdots \\
\cdots\end{array}$ & $\begin{array}{l}\cdots \\
\cdots \\
\cdots \\
\cdots\end{array}$ & $\begin{array}{l}\ldots \\
\ldots \\
\ldots \\
\cdots\end{array}$ & $\begin{array}{c}\cdots \\
\text { IV } \\
\cdots \\
\cdots \\
\cdots\end{array}$ & $\begin{array}{l}37 \\
\ldots \\
\ldots \\
\ldots\end{array}$ \\
\hline $\begin{array}{l}1964 \\
1964 \\
1964 \\
1964 \\
1964\end{array}$ & $\begin{array}{l}\text { MAY } \\
\text { MAY } \\
\text { JUN } \\
\text { JUN } \\
\text { JUL }\end{array}$ & $\begin{array}{l}22 \\
27 \\
05 \\
12 \\
01\end{array}$ & $\begin{array}{l}04 \\
17 \\
04 \\
03 \\
01\end{array}$ & $\begin{array}{l}56 \\
17 \\
52 \\
33 \\
11\end{array}$ & $\begin{array}{l}42 \\
23.3 \\
04.3 \\
35.9 \\
12.8\end{array}$ & $\begin{array}{l}44.6 \\
44.34 \\
43.2 \\
44.1 \\
42.60\end{array}$ & $\begin{array}{l}\text { N. } \\
\text { N. } \\
\text { N. } \\
\text { N. } \\
\text { N. }\end{array}$ & $\begin{array}{l}114.7 \\
114.73 \\
111.3 \\
114.7 \\
111.79\end{array}$ & $\begin{array}{l}w . \\
w . \\
w . \\
w . \\
w .\end{array}$ & $\begin{array}{l}005 \\
033 \\
015 \\
020\end{array}$ & $\begin{array}{l}D \\
C \\
D \\
C \\
D\end{array}$ & $\begin{array}{r}299 \\
260 \\
74 \\
266 \\
299\end{array}$ & $\begin{array}{c}\cdots \\
3.7 \\
\ldots \\
\ldots\end{array}$ & $\begin{array}{l}\cdots \\
\cdots \\
\cdots \\
\cdots\end{array}$ & $\begin{array}{l}\ldots \\
\cdots \\
\cdots \\
\cdots \\
\cdots\end{array}$ & $\begin{array}{l}\cdots \\
\cdots \\
\cdots \\
\cdots \\
\cdots\end{array}$ & $\begin{array}{l}\cdots \\
\cdots \\
\cdots \\
\cdots\end{array}$ & $\begin{array}{l}\cdots \\
\cdots \\
\cdots \\
\cdots \\
\cdots\end{array}$ & $\begin{array}{l}\cdots \\
\cdots \\
\cdots \\
\cdots \\
\cdots\end{array}$ & $\begin{array}{l}\cdots \\
\cdots \\
\cdots \\
\cdots\end{array}$ \\
\hline $\begin{array}{l}1964 \\
1964 \\
1964 \\
1964 \\
1964\end{array}$ & $\begin{array}{l}\text { JUL } \\
\text { JUL } \\
\text { AUG } \\
\text { SEP } \\
\text { SEP }\end{array}$ & $\begin{array}{l}01 \\
01 \\
10 \\
08 \\
12\end{array}$ & $\begin{array}{l}03 \\
19 \\
19 \\
00 \\
08\end{array}$ & $\begin{array}{l}41 \\
22 \\
18 \\
27 \\
45\end{array}$ & $\begin{array}{l}15.0 \\
36.8 \\
40 \\
55.5 \\
05.5\end{array}$ & $\begin{array}{l}42.6 \\
42.58 \\
44.3 \\
44.3 \\
44.2\end{array}$ & $\begin{array}{l}\text { N. } \\
\text { N. } \\
\text { N. } \\
\text { N. } \\
\text { N. }\end{array}$ & $\begin{array}{l}111.8 \\
111.91 \\
114.6 \\
114.8 \\
114.6\end{array}$ & $\begin{array}{l}w . \\
w . \\
w . \\
w . \\
w .\end{array}$ & $\begin{array}{r}033 \\
005 \\
033 \\
033\end{array}$ & $\begin{array}{l}D \\
C \\
D \\
C \\
C\end{array}$ & $\begin{array}{l}266 \\
260 \\
299 \\
266 \\
266\end{array}$ & $\begin{array}{c}\ldots \\
\ldots \\
3.9 \\
\ldots\end{array}$ & $\begin{array}{l}\cdots \\
\cdots \\
\cdots \\
\cdots\end{array}$ & $\begin{array}{l}\ldots \\
\ldots \\
\ldots \\
\ldots\end{array}$ & $\begin{array}{l}\cdots \\
\cdots \\
\cdots \\
\cdots \\
\cdots\end{array}$ & $\begin{array}{l}\cdots \\
\cdots \\
\cdots \\
\cdots\end{array}$ & $\begin{array}{l}\cdots \\
\cdots \\
\cdots \\
\cdots\end{array}$ & $\begin{array}{l}\cdots \\
\cdots \\
\cdots \\
\cdots \\
\cdots\end{array}$ & $\begin{array}{l}\cdots \\
\cdots \\
\cdots \\
\cdots\end{array}$ \\
\hline $\begin{array}{l}1964 \\
1964 \\
1964 \\
1964 \\
1964\end{array}$ & $\begin{array}{l}\text { SEP } \\
\text { SEP } \\
\text { OCT } \\
\text { OCT } \\
\text { OCT }\end{array}$ & $\begin{array}{l}22 \\
22 \\
15 \\
16 \\
28\end{array}$ & $\begin{array}{l}06 \\
08 \\
00 \\
18 \\
06\end{array}$ & $\begin{array}{l}52 \\
03 \\
37 \\
35 \\
42\end{array}$ & $\begin{array}{l}10.0 \\
51.0 \\
27 \\
53.2 \\
35\end{array}$ & $\begin{array}{l}44.4 \\
44.3 \\
44.2 \\
44.3 \\
44.0\end{array}$ & $\begin{array}{l}\text { N. } \\
\text { N. } \\
N . \\
N . \\
N .\end{array}$ & $\begin{array}{l}114.8 \\
114.7 \\
114.4 \\
114.6 \\
114.2\end{array}$ & $\begin{array}{l}w . \\
w . \\
w . \\
w . \\
w .\end{array}$ & $\begin{array}{l}015 \\
033 \\
033 \\
005 \\
033\end{array}$ & $\begin{array}{l}C \\
C \\
D \\
C \\
D\end{array}$ & $\begin{array}{r}74 \\
266 \\
299 \\
260 \\
299\end{array}$ & $\begin{array}{c}4.3 \\
\ldots \\
\ldots \\
\ldots \\
\ldots\end{array}$ & $\begin{array}{l}\cdots \\
\cdots \\
\cdots \\
\cdots\end{array}$ & $\begin{array}{l}\ldots \\
\ldots \\
\ldots \\
\ldots \\
\ldots\end{array}$ & $\begin{array}{l}\cdots \\
\cdots \\
\cdots \\
\cdots \\
\cdots\end{array}$ & $\begin{array}{l}\cdots \\
\cdots \\
\cdots \\
\cdots\end{array}$ & $\begin{array}{l}\cdots \\
\cdots \\
\cdots \\
\cdots \\
\cdots\end{array}$ & $\begin{array}{l}\cdots \\
\cdots \\
\cdots \\
\cdots \\
\cdots\end{array}$ & $\begin{array}{l}\cdots \\
\cdots \\
\cdots \\
\cdots\end{array}$ \\
\hline
\end{tabular}




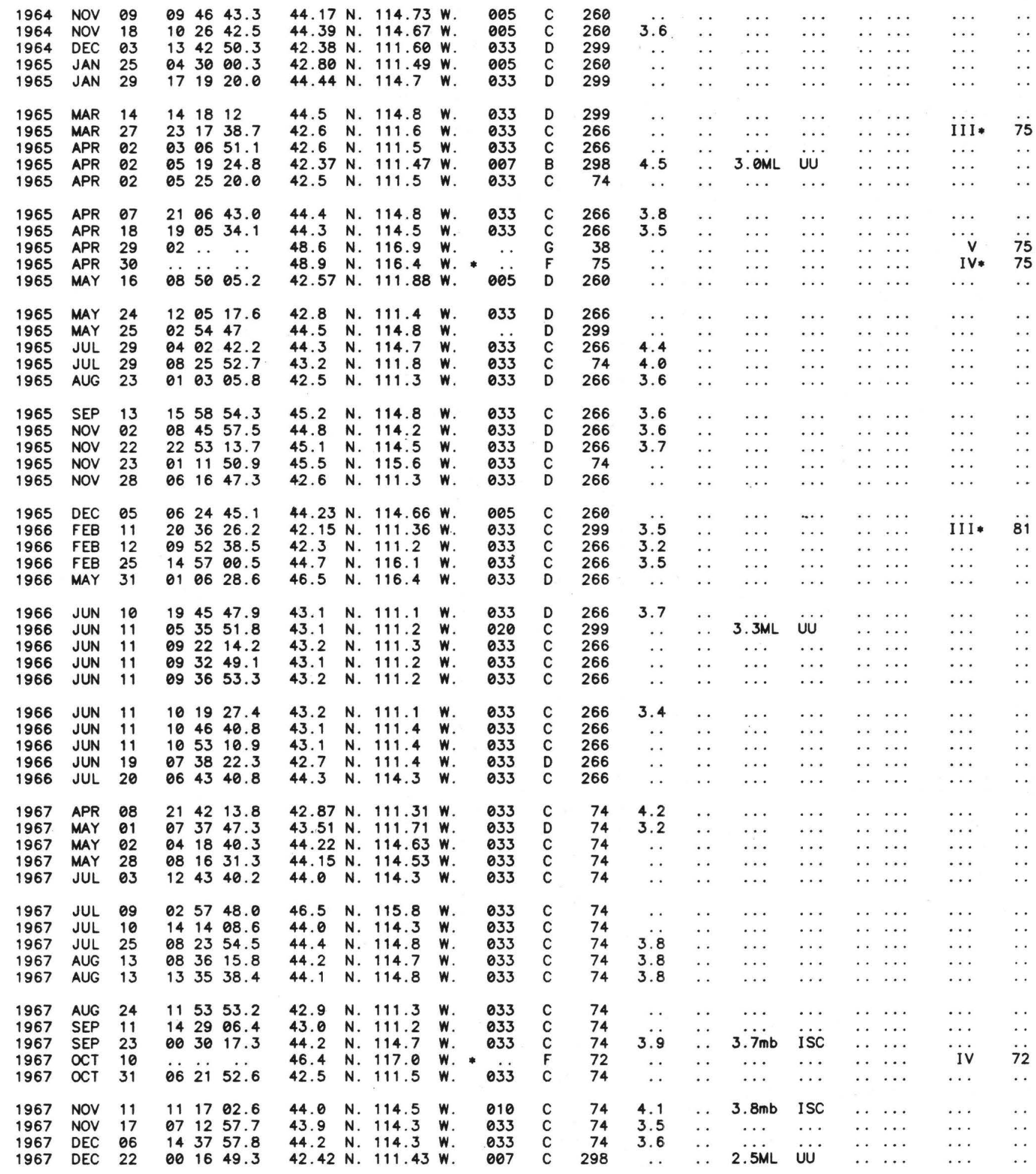




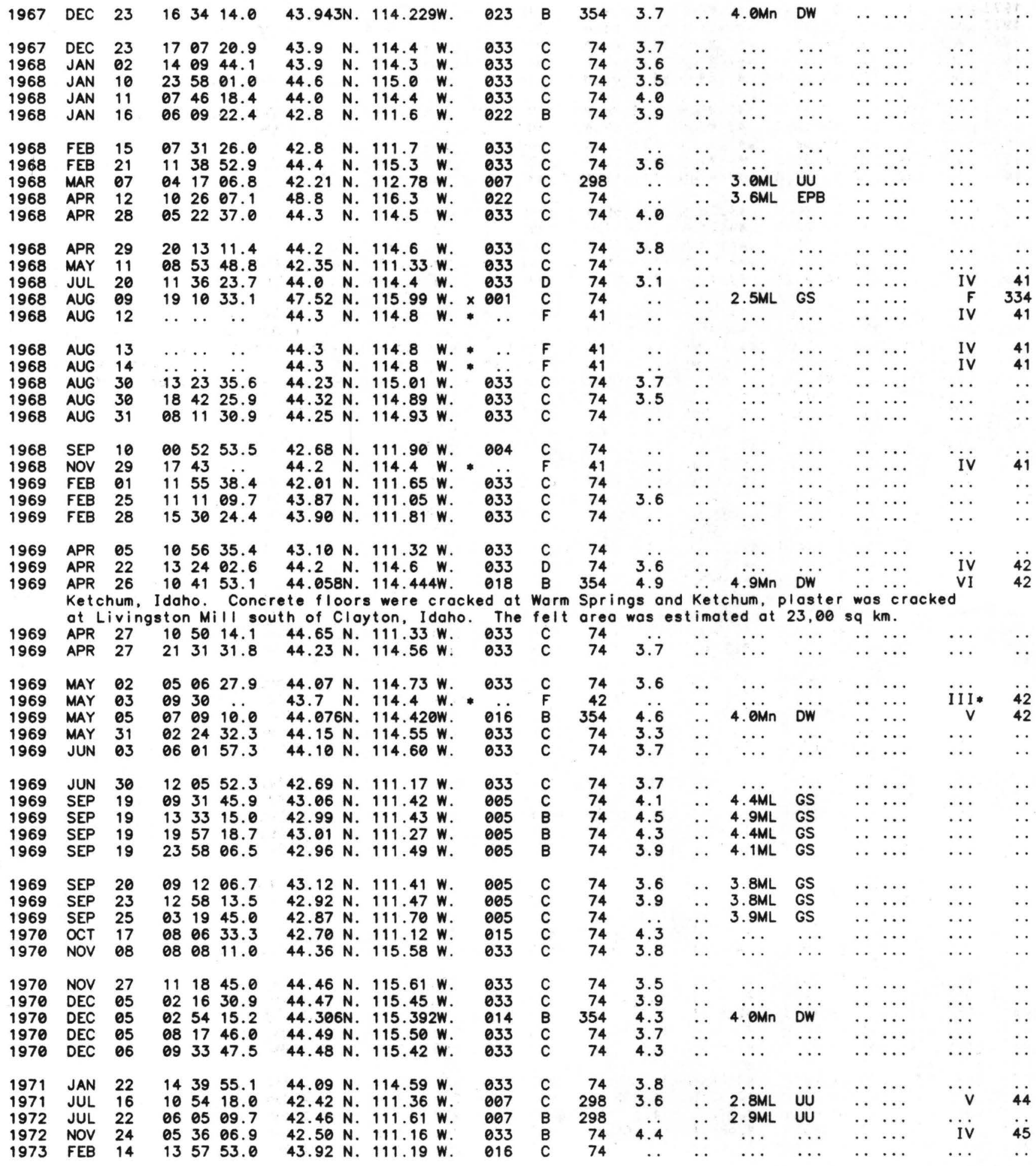




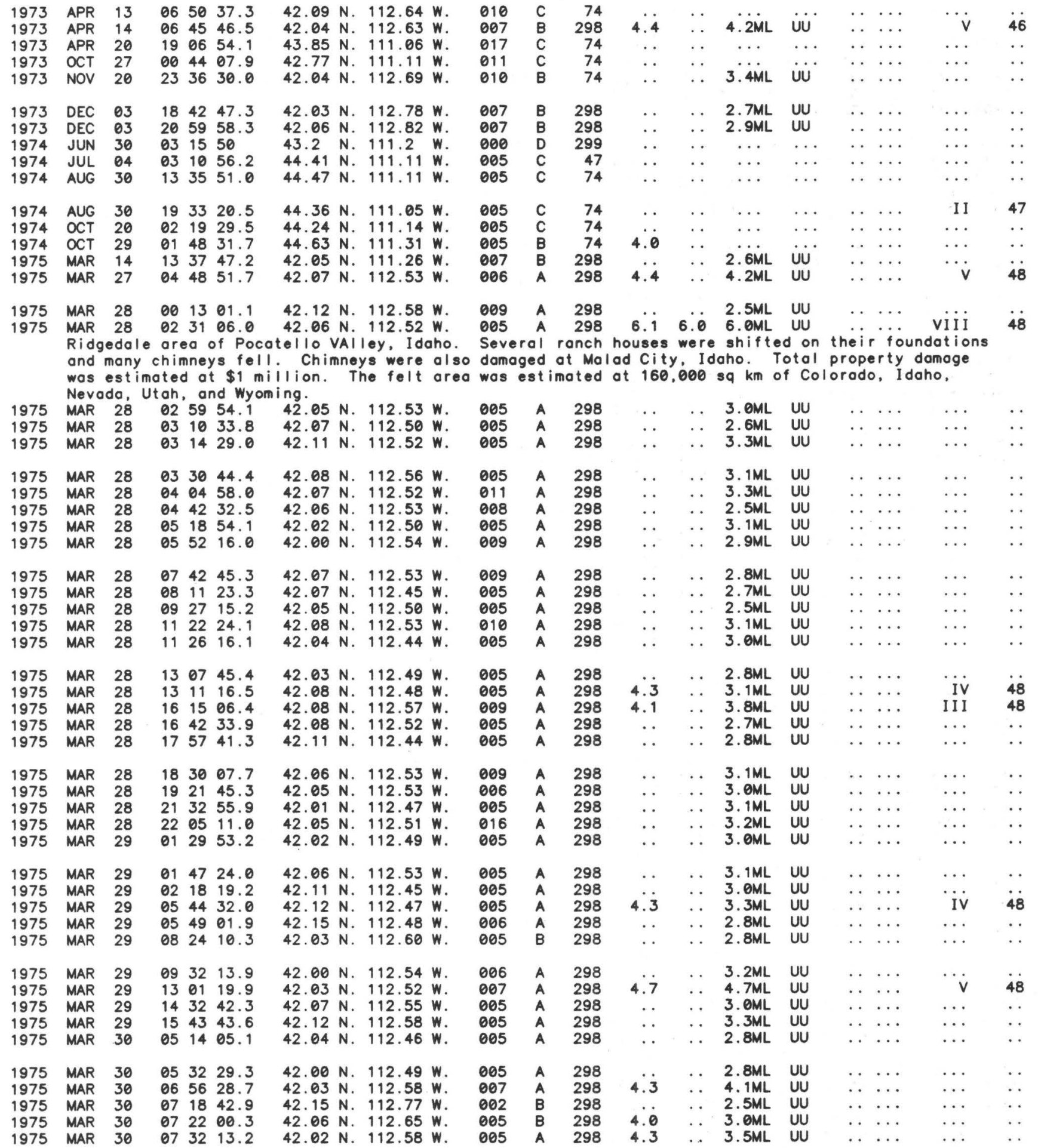




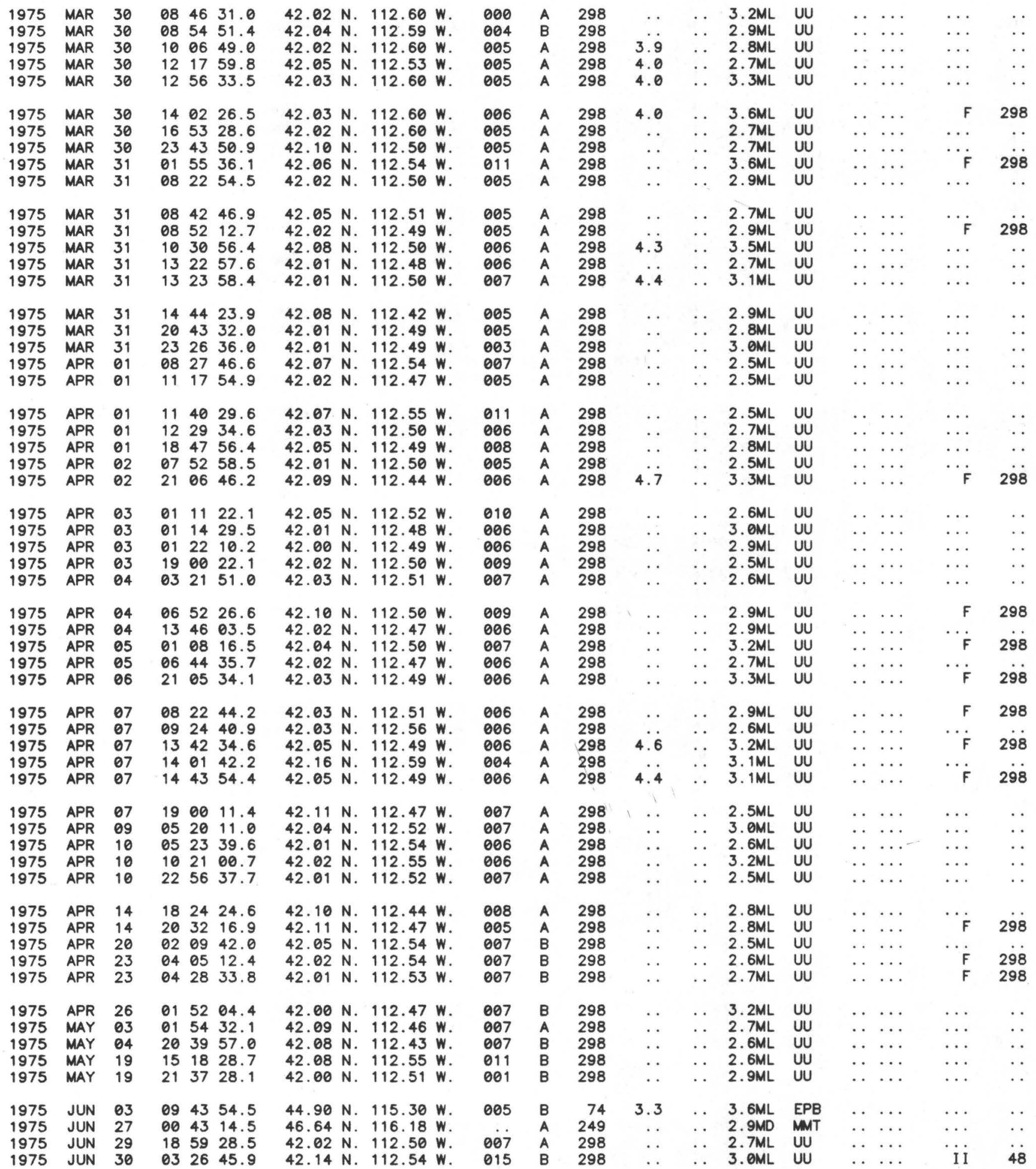




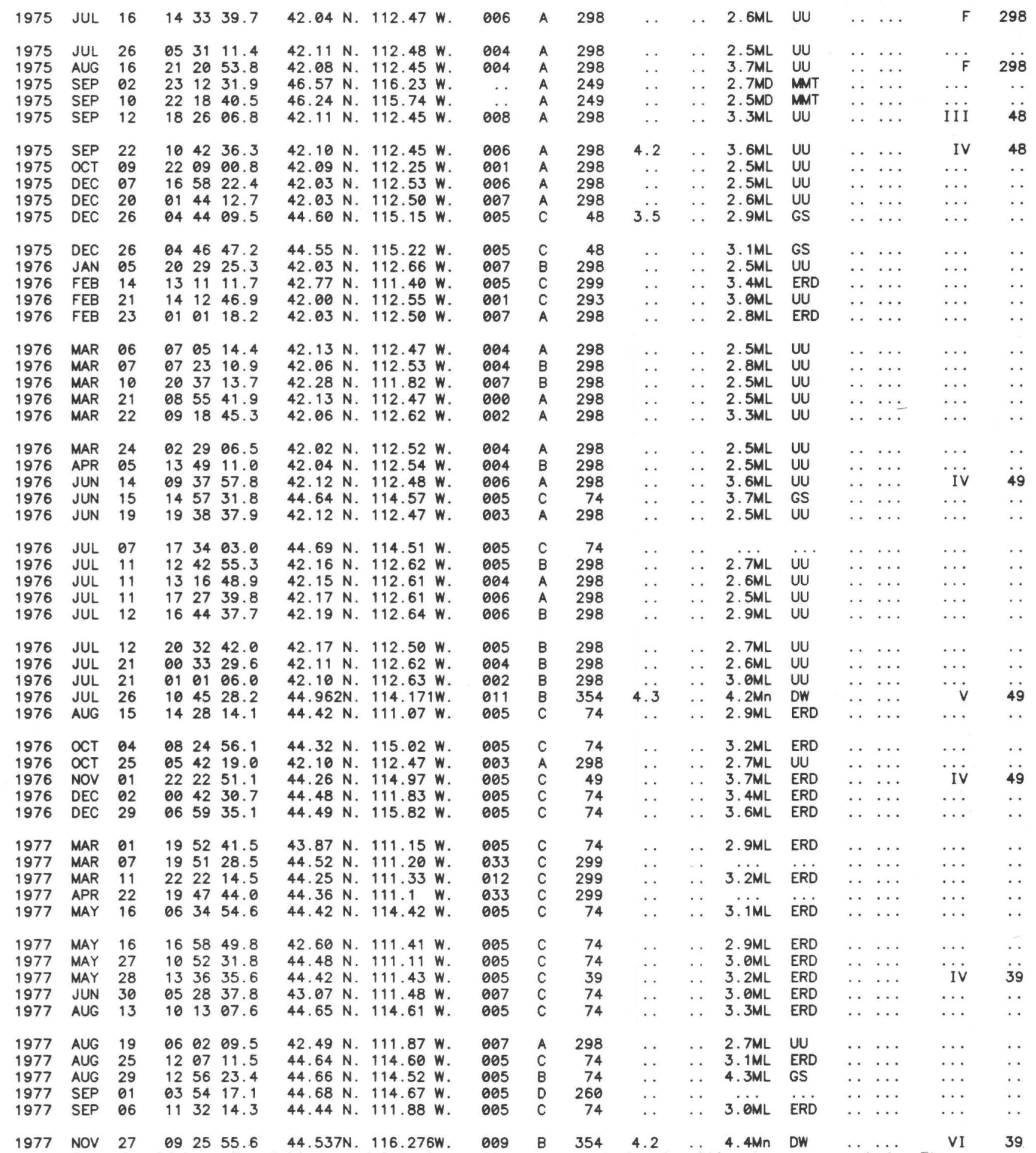
Cascade, Idaho. Foundations and interior walls were cracked and ceiling beams separated. The 
felt area was estimated at $24,000 \mathrm{sq} \mathrm{km}$ of Idaho and Oregon.

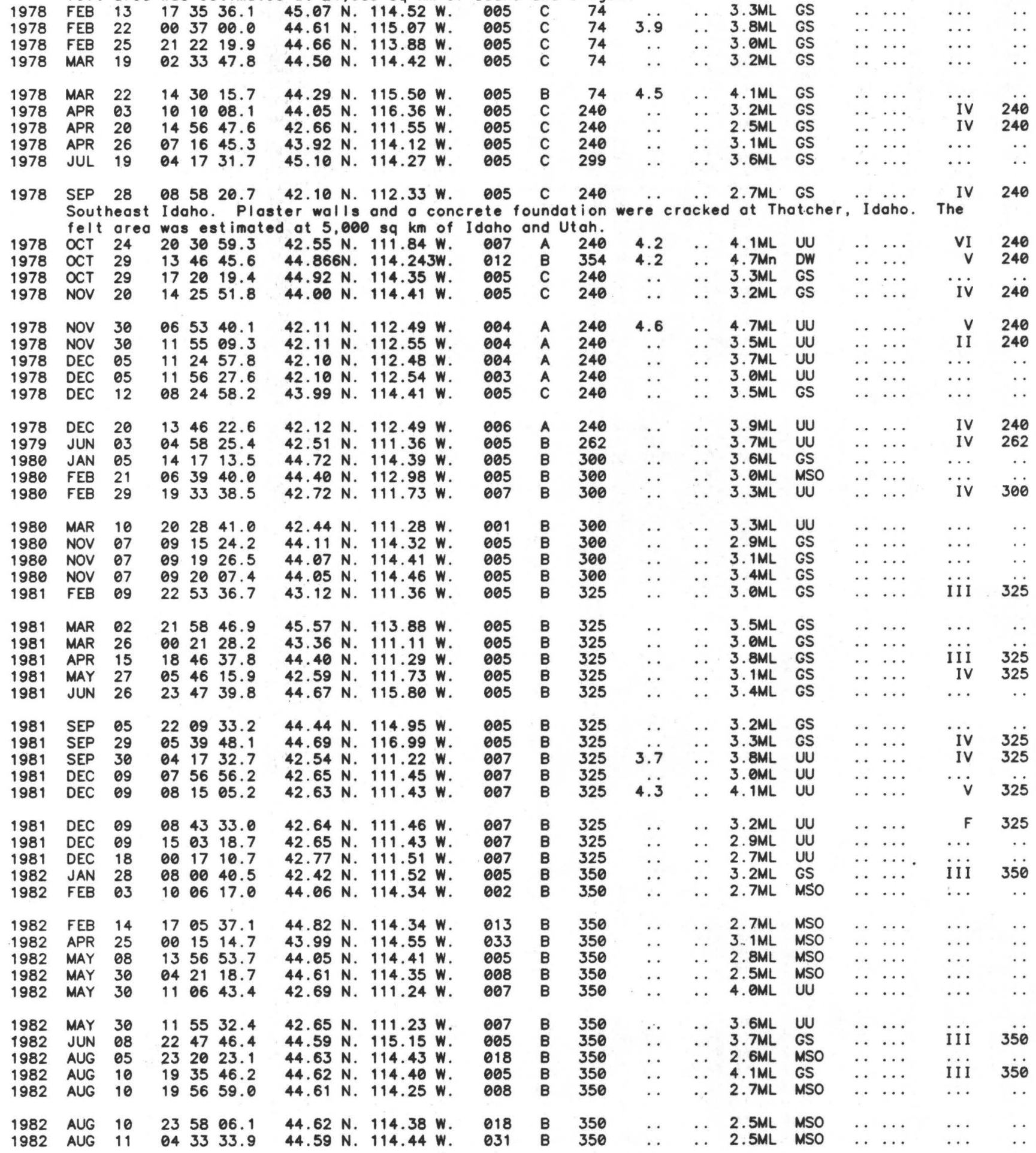




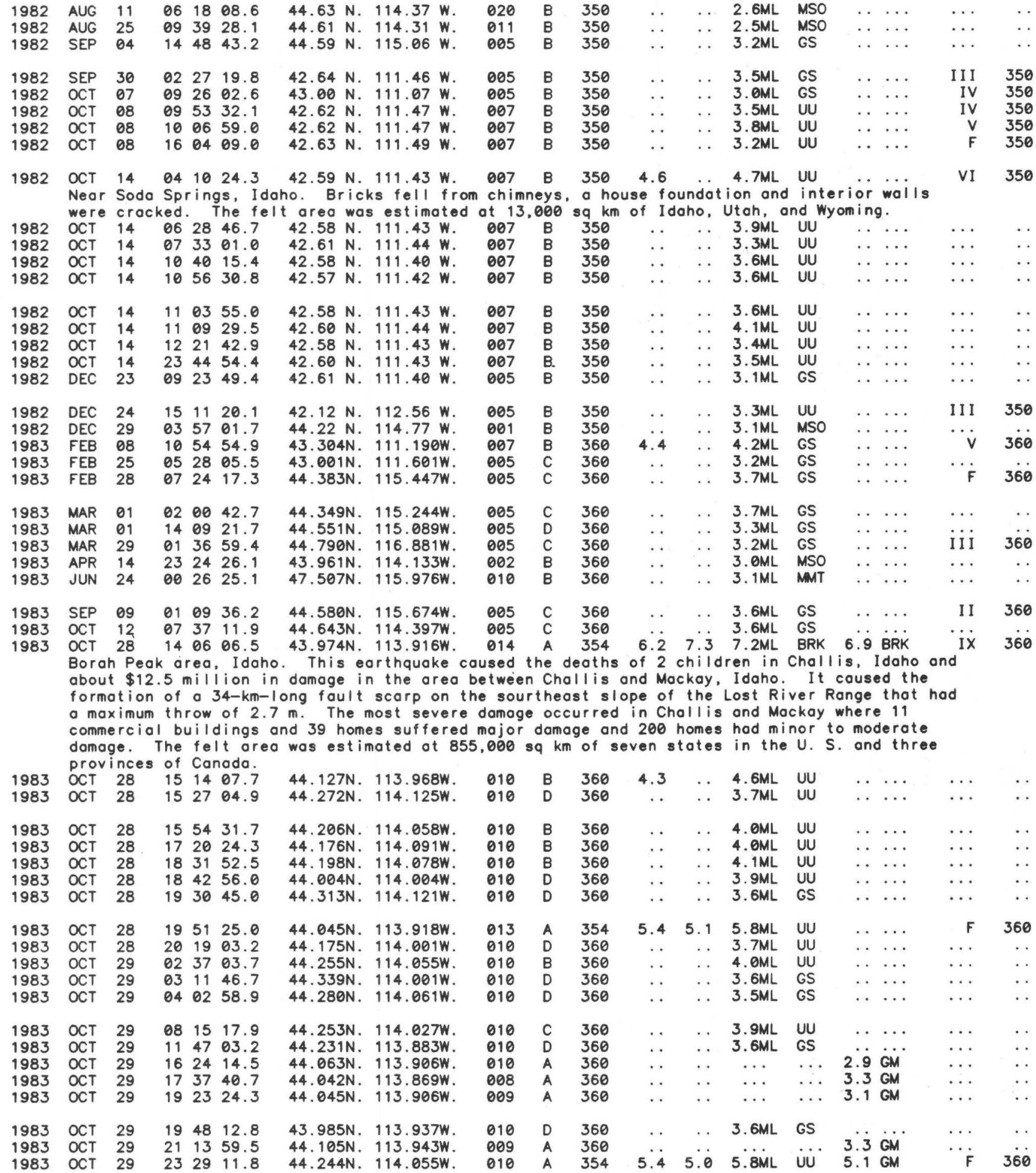




\begin{tabular}{|c|c|c|c|c|c|c|c|c|c|c|c|c|c|c|c|c|c|c|}
\hline $\begin{array}{l}1983 \\
1983\end{array}$ & $\begin{array}{l}\text { OCT } \\
\text { OCT }\end{array}$ & $\begin{array}{l}29 \\
29\end{array}$ & $\begin{array}{l}23 \\
23\end{array}$ & $\begin{array}{l}39 \\
49\end{array}$ & $\begin{array}{l}05.4 \\
43.6\end{array}$ & $\begin{array}{l}44.241 N \\
44.173 N\end{array}$ & $\begin{array}{l}114.109 w . \\
114.186 \mathrm{~W} .\end{array}$ & $\begin{array}{l}011 \\
010\end{array}$ & $\begin{array}{l}A \\
D\end{array}$ & $\begin{array}{l}354 \\
360\end{array}$ & $\begin{array}{r}5.5 \\
\ldots\end{array}$ & $\begin{array}{c}5.0 \\
\ldots\end{array}$ & $\begin{array}{l}\text { 5. } 4 \mathrm{ML} \\
\text { 3. } 8 \mathrm{ML}\end{array}$ & $\begin{array}{l}\text { UU } \\
\text { GS }\end{array}$ & $\begin{array}{r}4.7 \\
\ldots\end{array}$ & $\begin{array}{l}\text { GM } \\
\cdots\end{array}$ & $\begin{array}{r}F \\
\cdots\end{array}$ & $\begin{array}{r}360 \\
\ldots\end{array}$ \\
\hline $\begin{array}{l}1983 \\
1983 \\
1983 \\
1983 \\
1983\end{array}$ & $\begin{array}{l}\text { OCT } \\
\text { OCT } \\
\text { OCT } \\
\text { OCT } \\
\text { OCT }\end{array}$ & $\begin{array}{l}30 \\
30 \\
30 \\
30 \\
30\end{array}$ & $\begin{array}{l}01 \\
01 \\
01 \\
01 \\
02\end{array}$ & $\begin{array}{l}07 \\
16 \\
24 \\
59 \\
54\end{array}$ & $\begin{array}{l}41.3 \\
39.4 \\
51.3 \\
02.0 \\
39.7\end{array}$ & $\begin{array}{l}44.312 \mathrm{~N} \\
44.056 \mathrm{~N} \\
44.089 \mathrm{~N} \\
44.200 \mathrm{~N} \\
44.215 \mathrm{~N}\end{array}$ & $\begin{array}{l}114.068 w . \\
113.891 w . \\
113.977 w . \\
114.056 w . \\
114.115 w .\end{array}$ & $\begin{array}{l}010 \\
009 \\
013 \\
016 \\
006\end{array}$ & $\begin{array}{l}D \\
A \\
A \\
B \\
B\end{array}$ & $\begin{array}{l}360 \\
360 \\
360 \\
360 \\
360\end{array}$ & $\begin{array}{c}\ldots \\
4.3 \\
4.2 \\
\ldots\end{array}$ & $\begin{array}{l}\cdots \\
\cdots \\
\cdots \\
\cdots\end{array}$ & $\begin{array}{l}\text { 3. } 7 \mathrm{ML} \\
\ldots . \\
\text { 4. } 8 \mathrm{ML} \\
\text { 4. } 7 \mathrm{ML} \\
\text { 4. } 8 \mathrm{ML}\end{array}$ & $\begin{array}{l}\text { GS } \\
\text { UU } \\
\text { UU }\end{array}$ & $\begin{array}{l}3.3 \\
4.4 \\
4.2 \\
3.5\end{array}$ & $\begin{array}{l}\ddot{G M} \\
\text { GM } \\
\text { GM }\end{array}$ & $\begin{array}{l}\cdots \\
\cdots \\
\cdots \\
\cdots \\
\cdots\end{array}$ & $\begin{array}{l}\cdots \\
\cdots \\
\cdots \\
\cdots\end{array}$ \\
\hline $\begin{array}{l}1983 \\
1983 \\
1983 \\
1983 \\
1983\end{array}$ & $\begin{array}{l}\text { OCT } \\
\text { OCT } \\
\text { OCT } \\
\text { OCT } \\
\text { OCT }\end{array}$ & $\begin{array}{l}30 \\
30 \\
30 \\
30 \\
30\end{array}$ & $\begin{array}{l}03 \\
07 \\
09 \\
12 \\
12\end{array}$ & $\begin{array}{l}45 \\
14 \\
41 \\
25 \\
54\end{array}$ & $\begin{array}{l}19.3 \\
00.7 \\
33.1 \\
52.6 \\
00.0\end{array}$ & $\begin{array}{l}44.333 \mathrm{~N} \\
44.304 \mathrm{~N} \\
44.297 \mathrm{~N} \\
44.108 \mathrm{~N} \\
44.307 \mathrm{~N}\end{array}$ & $\begin{array}{l}113.829 W . \\
114.120 W . \\
114.112 W . \\
113.916 W . \\
114.084 W .\end{array}$ & $\begin{array}{l}002 \\
010 \\
010 \\
009 \\
010\end{array}$ & $\begin{array}{l}C \\
D \\
D \\
A \\
D\end{array}$ & $\begin{array}{l}360 \\
360 \\
360 \\
360 \\
360\end{array}$ & $\begin{array}{l}\cdots \\
\cdots \\
\cdots \\
\cdots\end{array}$ & $\begin{array}{l}\cdots \\
\cdots \\
\cdots \\
\cdots\end{array}$ & $\begin{array}{l}3.9 \mathrm{ML} \\
3.9 \mathrm{ML} \\
3.7 \mathrm{ML} \\
. .6 \mathrm{ML}\end{array}$ & $\begin{array}{l}\text { GS } \\
\text { GS } \\
\text { GS } \\
\ddot{G S}\end{array}$ & $\begin{array}{c}\cdots \\
\ldots \\
3 \\
\ldots\end{array}$ & $\begin{array}{l}\cdots \\
\cdots \\
\ddot{G M} \\
\cdots\end{array}$ & $\begin{array}{l}\cdots \\
\cdots \\
\cdots \\
\cdots \\
\cdots\end{array}$ & $\begin{array}{l}\cdots \\
\cdots \\
\cdots \\
\cdots\end{array}$ \\
\hline $\begin{array}{l}1983 \\
1983 \\
1983 \\
1983 \\
1983\end{array}$ & $\begin{array}{l}\text { OCT } \\
\text { OCT } \\
\text { OCT } \\
\text { OCT } \\
\text { OCT }\end{array}$ & $\begin{array}{l}30 \\
30 \\
30 \\
30 \\
31\end{array}$ & $\begin{array}{l}17 \\
19 \\
23 \\
23 \\
10\end{array}$ & $\begin{array}{l}49 \\
23 \\
02 \\
56 \\
33\end{array}$ & $\begin{array}{l}19.9 \\
42.4 \\
34.5 \\
26.0 \\
26.3\end{array}$ & $\begin{array}{l}44.157 \mathrm{~N} . \\
44.377 \mathrm{~N} . \\
44.026 \mathrm{~N} \\
44.255 \mathrm{~N} \\
44.184 \mathrm{~N}\end{array}$ & $\begin{array}{l}113.862 W . \\
113.994 W . \\
113.807 W . \\
114.081 W . \\
113.945 W .\end{array}$ & $\begin{array}{l}007 \\
010 \\
008 \\
006 \\
008\end{array}$ & $\begin{array}{l}\text { B } \\
D \\
A \\
B \\
A\end{array}$ & $\begin{array}{l}360 \\
360 \\
360 \\
360 \\
360\end{array}$ & $\begin{array}{l}\cdots \\
\cdots \\
\cdots \\
\cdots\end{array}$ & $\begin{array}{l}\cdots \\
\cdots \\
\cdots \\
\cdots\end{array}$ & $\begin{array}{c}3.8 \mathrm{ML} \\
3.6 \mathrm{ML} \\
\ldots \\
3.5 \mathrm{ML} \\
\ldots\end{array}$ & $\begin{array}{l}\text { GS } \\
\text { GS } \\
\dot{G S} \\
\ldots\end{array}$ & $\begin{array}{r}3.7 \\
3.1 \\
3.2 \\
3.0\end{array}$ & $\begin{array}{l}\text { GM } \\
\ddot{G M} \\
\text { GM }\end{array}$ & $\begin{array}{l}\cdots \\
\cdots \\
\cdots \\
\cdots \\
\cdots\end{array}$ & $\begin{array}{l}\ldots \\
\cdots \\
\cdots \\
\cdots\end{array}$ \\
\hline $\begin{array}{l}1983 \\
1983 \\
1983 \\
1983 \\
1983\end{array}$ & $\begin{array}{l}\text { OCT } \\
\text { NOV } \\
\text { NOV } \\
\text { NOV } \\
\text { NOV }\end{array}$ & $\begin{array}{l}31 \\
01 \\
01 \\
01 \\
01\end{array}$ & $\begin{array}{l}16 \\
01 \\
05 \\
10 \\
13\end{array}$ & $\begin{array}{l}08 \\
05 \\
02 \\
30 \\
50\end{array}$ & $\begin{array}{l}33.3 \\
28.2 \\
46.6 \\
33.6 \\
25.0\end{array}$ & $\begin{array}{l}44.258 \mathrm{~N} \\
44.232 \mathrm{~N} \\
44.052 \mathrm{~N} \\
44.134 \mathrm{~N} \\
44.152 \mathrm{~N}\end{array}$ & $\begin{array}{l}113.990 w . \\
114.053 w . \\
113.889 w . \\
113.950 w . \\
113.986 W .\end{array}$ & $\begin{array}{l}010 \\
008 \\
011 \\
010 \\
012\end{array}$ & $\begin{array}{l}D \\
A \\
A \\
A \\
A\end{array}$ & $\begin{array}{l}360 \\
360 \\
360 \\
360 \\
360\end{array}$ & $\begin{array}{l}\cdots \\
\cdots \\
\cdots \\
\cdots\end{array}$ & $\begin{array}{l}\cdots \\
\cdots \\
\cdots \\
\cdots\end{array}$ & $\begin{array}{c}3.6 \mathrm{ML} \\
\ldots \\
\ldots \\
3.7 \mathrm{ML}\end{array}$ & $\begin{array}{l}\text { GS } \\
\ldots \\
\cdots \\
\ddot{U}\end{array}$ & $\begin{array}{l}3.1 \\
2.7 \\
2.9 \\
3.1\end{array}$ & $\begin{array}{l}\ddot{G M} \\
\text { GM } \\
\text { GM }\end{array}$ & $\begin{array}{l}\cdots \\
\cdots \\
\cdots \\
\cdots \\
\cdots\end{array}$ & $\begin{array}{l}\cdots \\
\cdots \\
\cdots\end{array}$ \\
\hline $\begin{array}{l}1983 \\
1983 \\
1983 \\
1983 \\
1983\end{array}$ & $\begin{array}{l}\text { NOV } \\
\text { NOV } \\
\text { NOV } \\
\text { NOV } \\
\text { NOV }\end{array}$ & $\begin{array}{l}02 \\
02 \\
02 \\
02 \\
03\end{array}$ & $\begin{array}{l}12 \\
22 \\
23 \\
23 \\
00\end{array}$ & $\begin{array}{l}41 \\
24 \\
42 \\
43 \\
18\end{array}$ & $\begin{array}{l}12.9 \\
04.6 \\
01.9 \\
55.1 \\
48.4\end{array}$ & $\begin{array}{l}44.246 \mathrm{~N} \\
44.232 \mathrm{~N} \\
44.265 \mathrm{~N} \\
44.260 \mathrm{~N} \\
44.259 \mathrm{~N}\end{array}$ & $\begin{array}{l}114.158 W . \\
114.057 W . \\
114.082 W . \\
114.081 W . \\
114.036 w .\end{array}$ & $\begin{array}{l}010 \\
009 \\
006 \\
006 \\
009\end{array}$ & $\begin{array}{l}\text { C } \\
A \\
A \\
A \\
A\end{array}$ & $\begin{array}{l}360 \\
360 \\
360 \\
360 \\
360\end{array}$ & $\begin{array}{l}\cdots \\
\cdots \\
\cdots \\
\cdots\end{array}$ & $\begin{array}{l}\cdots \\
\cdots \\
\cdots \\
\cdots\end{array}$ & $\begin{array}{c}3.7 \mathrm{ML} \\
\ldots \\
\ldots \\
4.2 \mathrm{ML} \\
\ldots\end{array}$ & $\begin{array}{l}\text { GS } \\
\ldots \\
\ddot{U} \\
\cdots\end{array}$ & $\begin{array}{l}3.0 \\
3.3 \\
4.1 \\
3.3\end{array}$ & $\begin{array}{l}\ddot{G M} \\
\text { GM } \\
\text { GM } \\
\text { GM }\end{array}$ & $\begin{array}{l}\cdots \\
\cdots \\
\cdots \\
\cdots \\
\cdots\end{array}$ & $\begin{array}{l}\cdots \\
\cdots \\
\cdots \\
\cdots\end{array}$ \\
\hline $\begin{array}{l}1983 \\
1983 \\
1983 \\
1983 \\
1983\end{array}$ & $\begin{array}{l}\text { NOV } \\
\text { NOV } \\
\text { NOV } \\
\text { NOV } \\
\text { NOV }\end{array}$ & $\begin{array}{l}03 \\
03 \\
03 \\
03 \\
03\end{array}$ & $\begin{array}{l}01 \\
01 \\
02 \\
02 \\
04\end{array}$ & $\begin{array}{l}22 \\
50 \\
24 \\
59 \\
15\end{array}$ & $\begin{array}{l}15.7 \\
20.3 \\
13.6 \\
19.2 \\
16.4\end{array}$ & $\begin{array}{l}44.262 \mathrm{~N} \\
44.249 \mathrm{~N} \\
44.377 \mathrm{~N} \\
44.228 \mathrm{~N} \\
44.253 \mathrm{~N}\end{array}$ & $\begin{array}{l}114.065 w . \\
114.080 W . \\
114.165 w . \\
114.031 W . \\
114.141 W .\end{array}$ & $\begin{array}{l}008 \\
007 \\
010 \\
010 \\
008\end{array}$ & $\begin{array}{l}A \\
A \\
C \\
A \\
A\end{array}$ & $\begin{array}{l}360 \\
360 \\
360 \\
360 \\
360\end{array}$ & $\begin{array}{c}\ldots \\
\ldots \\
\cdots \\
\cdots\end{array}$ & $\begin{array}{l}\cdots \\
\cdots \\
\cdots \\
\cdots\end{array}$ & $\begin{array}{l}3.8 \mathrm{ML} \\
3.4 \mathrm{ML} \\
3.9 \mathrm{ML} \\
3.6 \mathrm{ML}\end{array}$ & $\begin{array}{l}\ddot{U U} \\
\text { GS } \\
\text { UU }\end{array}$ & $\begin{array}{r}3.2 \\
3.6 \\
3.6 \\
3.6\end{array}$ & $\begin{array}{l}\text { GM } \\
\text { GM } \\
\ddot{G M} \\
\text { GM }\end{array}$ & $\begin{array}{l}\cdots \\
\cdots \\
\cdots \\
\cdots \\
\cdots\end{array}$ & $\begin{array}{l}\cdots \\
\ldots \\
\cdots \\
\cdots\end{array}$ \\
\hline $\begin{array}{l}1983 \\
1983 \\
1983 \\
1983 \\
1983\end{array}$ & $\begin{array}{l}\text { NOV } \\
\text { NOV } \\
\text { NOV } \\
\text { NOV } \\
\text { NOV }\end{array}$ & $\begin{array}{l}03 \\
03 \\
03 \\
03 \\
03\end{array}$ & $\begin{array}{l}04 \\
14 \\
15 \\
17 \\
22\end{array}$ & $\begin{array}{l}47 \\
14 \\
47 \\
00 \\
09\end{array}$ & $\begin{array}{l}35.6 \\
17.8 \\
30.1 \\
14.0 \\
24.5\end{array}$ & $\begin{array}{l}44.394 \mathrm{~N} \\
44.308 \mathrm{~N} \\
44.303 \mathrm{~N} \\
43.885 \mathrm{~N} \\
44.101 \mathrm{~N}\end{array}$ & $\begin{array}{l}114.074 W . \\
114.142 W . \\
114.052 W . \\
113.679 W . \\
113.951 W .\end{array}$ & $\begin{array}{l}010 \\
010 \\
010 \\
008 \\
011\end{array}$ & $\begin{array}{l}\text { C } \\
\text { C } \\
\text { C } \\
A \\
A\end{array}$ & $\begin{array}{l}360 \\
360 \\
360 \\
360 \\
360\end{array}$ & $\begin{array}{l}\cdots \\
\cdots \\
\cdots \\
\cdots\end{array}$ & $\begin{array}{l}\cdots \\
\cdots \\
\cdots \\
\cdots\end{array}$ & $\begin{array}{c}3.5 \mathrm{ML} \\
3.6 \mathrm{ML} \\
3.6 \mathrm{ML} \\
\ldots \\
\ldots\end{array}$ & $\begin{array}{l}\text { GS } \\
\text { GS } \\
\text { GS } \\
\cdots \\
\cdots\end{array}$ & $\begin{array}{c}\cdots \\
\cdots \\
2.7 \\
2.7\end{array}$ & $\begin{array}{l}\cdots \\
\cdots \\
\cdots \\
\mathrm{GM}\end{array}$ & $\begin{array}{l}\cdots \\
\cdots \\
\cdots \\
\cdots \\
\cdots\end{array}$ & $\begin{array}{l}\cdots \\
\cdots \\
\cdots \\
\cdots\end{array}$ \\
\hline $\begin{array}{l}1983 \\
1983 \\
1983 \\
1983 \\
1983\end{array}$ & $\begin{array}{l}\text { NOV } \\
\text { NOV } \\
\text { NOV } \\
\text { NOV } \\
\text { NOV }\end{array}$ & $\begin{array}{l}04 \\
04 \\
04 \\
04 \\
04\end{array}$ & $\begin{array}{l}00 \\
05 \\
07 \\
09 \\
13\end{array}$ & $\begin{array}{l}02 \\
00 \\
08 \\
04 \\
43\end{array}$ & $\begin{array}{l}25.5 \\
14.8 \\
19.2 \\
12.9 \\
01.1\end{array}$ & $\begin{array}{l}44.177 N \\
44.139 \mathrm{~N} \\
44.207 \mathrm{~N} \\
44.139 \mathrm{~N} \\
44.200 \mathrm{~N}\end{array}$ & $\begin{array}{l}114.013 W . \\
113.938 W . \\
114.024 W . \\
113.912 W . \\
114.029 W .\end{array}$ & $\begin{array}{l}011 \\
009 \\
009 \\
007 \\
009\end{array}$ & $\begin{array}{l}A \\
A \\
A \\
A \\
A\end{array}$ & $\begin{array}{l}360 \\
360 \\
360 \\
360 \\
360\end{array}$ & $\begin{array}{l}\cdots \\
\cdots \\
\cdots \\
\cdots\end{array}$ & $\begin{array}{l}\cdots \\
\cdots \\
\cdots \\
\cdots\end{array}$ & $\begin{array}{c}3 . \overline{\mathrm{ML}} \\
3.5 \mathrm{ML} \\
\ldots \\
\ldots\end{array}$ & $\begin{array}{l}\ddot{\mathrm{GS}} \\
\mathrm{GS} \\
\cdots \\
\cdots\end{array}$ & $\begin{array}{l}2.5 \\
3.0 \\
3.4 \\
3.1 \\
2.9\end{array}$ & $\begin{array}{l}\text { GM } \\
G M \\
G M \\
G M \\
G M\end{array}$ & $\begin{array}{l}\cdots \\
\cdots \\
\cdots \\
\cdots \\
\cdots\end{array}$ & $\begin{array}{l}\cdots \\
\cdots \\
\cdots \\
\cdots\end{array}$ \\
\hline $\begin{array}{l}1983 \\
1983 \\
1983 \\
1983 \\
1983\end{array}$ & $\begin{array}{l}\text { NOV } \\
\text { NOV } \\
\text { NOV } \\
\text { NOV } \\
\text { NOV }\end{array}$ & $\begin{array}{l}04 \\
04 \\
05 \\
05 \\
05\end{array}$ & $\begin{array}{l}23 \\
23 \\
01 \\
03 \\
04\end{array}$ & $\begin{array}{l}29 \\
36 \\
51 \\
53 \\
22\end{array}$ & $\begin{array}{l}49.3 \\
45.4 \\
49.1 \\
34.3 \\
09.0\end{array}$ & $\begin{array}{l}44.257 \mathrm{~N} \\
44.237 \mathrm{~N} \\
44.131 \mathrm{~N} . \\
44.153 \mathrm{~N} \\
44.141 \mathrm{~N}\end{array}$ & $\begin{array}{l}114.096 \mathrm{~W} . \\
114.072 \mathrm{~W} . \\
113.951 \mathrm{~W} . \\
113.895 \mathrm{~W} . \\
113.970 \mathrm{~W} .\end{array}$ & $\begin{array}{l}008 \\
008 \\
012 \\
006 \\
010\end{array}$ & $\begin{array}{l}\text { A } \\
A \\
A \\
A \\
A\end{array}$ & $\begin{array}{l}360 \\
360 \\
360 \\
360 \\
360\end{array}$ & $\begin{array}{l}\cdots \\
\cdots \\
\cdots \\
\cdots\end{array}$ & $\begin{array}{l}\cdots \\
\cdots \\
\cdots \\
\cdots\end{array}$ & $\begin{array}{l}\cdots \\
\cdots \\
\cdots \\
\cdots \\
\cdots\end{array}$ & $\begin{array}{l}\cdots \\
\cdots \\
\cdots \\
\cdots \\
\cdots\end{array}$ & $\begin{array}{l}3.2 \\
2.7 \\
2.9 \\
2.8 \\
2.9\end{array}$ & $\begin{array}{l}\text { GM } \\
\text { GM } \\
\text { GM } \\
\text { GM } \\
\text { GM }\end{array}$ & $\begin{array}{l}\cdots \\
\cdots \\
\cdots \\
\cdots \\
\cdots\end{array}$ & $\begin{array}{l}\cdots \\
\cdots \\
\cdots \\
\cdots\end{array}$ \\
\hline $\begin{array}{l}1983 \\
1983 \\
1983 \\
1983 \\
1983\end{array}$ & $\begin{array}{l}\text { NOV } \\
\text { NOV } \\
\text { NOV } \\
\text { NOV } \\
\text { NOV }\end{array}$ & $\begin{array}{l}05 \\
05 \\
05 \\
05 \\
05\end{array}$ & $\begin{array}{l}05 \\
08 \\
17 \\
17 \\
20\end{array}$ & $\begin{array}{l}37 \\
13 \\
36 \\
43 \\
29\end{array}$ & $\begin{array}{l}39.8 \\
39.4 \\
25.3 \\
54.7 \\
30.1\end{array}$ & $\begin{array}{l}44.227 \mathrm{~N} \\
44.206 \mathrm{~N} \\
44.181 \mathrm{~N} \\
44.144 \mathrm{~N} \\
44.197 \mathrm{~N}\end{array}$ & $\begin{array}{l}114.067 W . \\
114.023 W . \\
114.023 W . \\
113.987 W . \\
114.030 W .\end{array}$ & $\begin{array}{l}010 \\
008 \\
012 \\
014 \\
010\end{array}$ & $\begin{array}{l}A \\
A \\
A \\
A \\
A\end{array}$ & $\begin{array}{l}360 \\
360 \\
360 \\
360 \\
360\end{array}$ & $\begin{array}{l}\cdots \\
\cdots \\
\cdots \\
\cdots\end{array}$ & $\begin{array}{l}\cdots \\
\cdots \\
\cdots \\
\cdots\end{array}$ & $\begin{array}{c}3.6 \mathrm{ML} \\
\ldots \\
3.5 \mathrm{ML} \\
\ldots \\
\ldots\end{array}$ & $\begin{array}{l}\mathrm{GS} \\
\cdots \\
\mathrm{GS} \\
\cdots\end{array}$ & $\begin{array}{l}3.5 \\
2.8 \\
3.4 \\
3.1 \\
3.2\end{array}$ & $\begin{array}{l}\text { GM } \\
\text { GM } \\
\text { GM } \\
\text { GM } \\
\text { GM }\end{array}$ & $\begin{array}{l}\cdots \\
\cdots \\
\cdots \\
\cdots \\
\cdots\end{array}$ & $\begin{array}{l}\cdots \\
\cdots \\
\cdots \\
\cdots\end{array}$ \\
\hline $\begin{array}{l}1983 \\
1983 \\
1983 \\
1983 \\
1983\end{array}$ & $\begin{array}{l}\text { NOV } \\
\text { NOV } \\
\text { NOV } \\
\text { NOV } \\
\text { NOV }\end{array}$ & $\begin{array}{l}05 \\
06 \\
06 \\
06 \\
06\end{array}$ & $\begin{array}{l}22 \\
03 \\
06 \\
21 \\
21\end{array}$ & $\begin{array}{l}56 \\
26 \\
44 \\
04 \\
11\end{array}$ & $\begin{array}{l}42.7 \\
09.4 \\
15.9 \\
48.7 \\
55.0\end{array}$ & $\begin{array}{l}44.222 \mathrm{~N} \\
44.262 \mathrm{~N} \\
44.171 \mathrm{~N} \\
44.140 \mathrm{~N} \\
44.139 \mathrm{~N}\end{array}$ & $\begin{array}{l}114.039 w . \\
114.085 W . \\
113.972 W . \\
113.963 W . \\
113.977 W .\end{array}$ & $\begin{array}{l}012 \\
007 \\
011 \\
011 \\
011\end{array}$ & $\begin{array}{l}A \\
A \\
A \\
A \\
B\end{array}$ & $\begin{array}{l}360 \\
360 \\
360 \\
360 \\
360\end{array}$ & $\begin{array}{c}\cdots \\
\cdots \\
4 \dot{3} \\
\cdots\end{array}$ & $\begin{array}{l}\cdots \\
\cdots \\
\cdots \\
\cdots\end{array}$ & $\begin{array}{c}\cdots \\
\cdots \\
4.6 \mathrm{ML} \\
3.8 \mathrm{ML}\end{array}$ & $\begin{array}{l}\cdots \\
\cdots \\
\text { UU }\end{array}$ & $\begin{array}{l}3.2 \\
2.9 \\
3.0 \\
4.2 \\
3.5\end{array}$ & $\begin{array}{l}\text { GM } \\
\text { GM } \\
\text { GM } \\
\text { GM } \\
\text { GM }\end{array}$ & $\begin{array}{l}\cdots \\
\cdots \\
\text { III } \\
\cdots\end{array}$ & $\begin{array}{c}\cdots \\
\cdots \\
360 \\
\cdots\end{array}$ \\
\hline 1983 & NOV & 06 & 21 & 19 & 56.4 & $44.146 \mathrm{~N}$ & $113.974 W$ & 010 & A & 360 & . & $\cdots$ & $\cdots$ & $\cdots$ & 3.0 & GM & $\cdots$ & $\cdots$ \\
\hline
\end{tabular}




\begin{tabular}{|c|c|c|c|c|c|c|c|c|c|c|c|c|c|c|c|c|c|c|}
\hline $\begin{array}{l}1983 \\
1983 \\
1983 \\
1983\end{array}$ & $\begin{array}{l}\text { NOV } \\
\text { NOV } \\
\text { NOV } \\
\text { NOV }\end{array}$ & $\begin{array}{l}06 \\
06 \\
07 \\
07\end{array}$ & $\begin{array}{l}21 \\
23 \\
09 \\
17\end{array}$ & $\begin{array}{l}20 \\
11 \\
33 \\
33\end{array}$ & $\begin{array}{l}40.2 \\
38.6 \\
31.2 \\
06.1\end{array}$ & $\begin{array}{l}44.142 \mathrm{~N} . \\
44.227 \mathrm{~N} . \\
44.170 \mathrm{~N} . \\
44.257 \mathrm{~N}\end{array}$ & $\begin{array}{l}113.975 w . \\
114.044 W . \\
113.968 w . \\
114.046 w .\end{array}$ & $\begin{array}{l}010 \\
007 \\
009 \\
010\end{array}$ & $\begin{array}{l}A \\
A \\
A \\
A\end{array}$ & $\begin{array}{l}360 \\
360 \\
360 \\
360\end{array}$ & $\begin{array}{l}\cdots \\
\cdots \\
\cdots\end{array}$ & $\begin{array}{l}\cdots \\
\cdots \\
\cdots\end{array}$ & $\begin{array}{l}\cdots \\
\cdots \\
\cdots \\
\cdots\end{array}$ & $\begin{array}{l}\cdots \\
\cdots \\
\cdots \\
\cdots\end{array}$ & $\begin{array}{l}3.1 \\
3.1 \\
3.2 \\
3.1\end{array}$ & $\begin{array}{l}\text { GM } \\
G M \\
G M \\
G M\end{array}$ & $\begin{array}{l}\cdots \\
\cdots \\
\cdots \\
\cdots\end{array}$ & $\begin{array}{l}\cdots \\
\cdots \\
\cdots\end{array}$ \\
\hline $\begin{array}{l}1983 \\
1983 \\
1983 \\
1983 \\
1983\end{array}$ & $\begin{array}{l}\text { NOV } \\
\text { NOV } \\
\text { NOV } \\
\text { NOV } \\
\text { NOV }\end{array}$ & $\begin{array}{l}08 \\
08 \\
09 \\
09 \\
19\end{array}$ & $\begin{array}{l}06 \\
23 \\
09 \\
23 \\
03\end{array}$ & $\begin{array}{l}43 \\
32 \\
25 \\
00 \\
50\end{array}$ & $\begin{array}{l}02.5 \\
43.0 \\
04.3 \\
43.3 \\
46.9\end{array}$ & $\begin{array}{l}44.168 \mathrm{~N} \\
44.236 \mathrm{~N} \\
44.480 \mathrm{~N} \\
43.897 \mathrm{~N} \\
42.055 \mathrm{~N}\end{array}$ & $\begin{array}{l}113.958 W . \\
114.060 w . \\
114.140 W . \\
113.755 W . \\
112.499 w .\end{array}$ & $\begin{array}{l}009 \\
007 \\
010 \\
011 \\
005\end{array}$ & $\begin{array}{l}A \\
B \\
C \\
B \\
B\end{array}$ & $\begin{array}{l}360 \\
360 \\
360 \\
360 \\
360\end{array}$ & $\begin{array}{l}\cdots \\
\cdots \\
\cdots \\
\cdots\end{array}$ & $\begin{array}{l}\cdots \\
\cdots \\
\cdots \\
\cdots\end{array}$ & $\begin{array}{l}3.5 \mathrm{ML} \\
3.4 \mathrm{ML} \\
3.4 \mathrm{ML} \\
3.8 \mathrm{ML}\end{array}$ & $\begin{array}{l}\ddot{G S} \\
\text { GS } \\
\text { GS } \\
\text { UU }\end{array}$ & $\begin{array}{c}2.9 \\
\ldots \\
\ldots \\
\ldots\end{array}$ & $\begin{array}{l}\mathrm{GM} \\
\ldots \\
\ldots \\
\ldots \\
\ldots\end{array}$ & $\begin{array}{l}\cdots \\
\cdots \\
\cdots \\
\cdots \\
v\end{array}$ & $\begin{array}{c}\cdots \\
\cdots \\
\cdots \\
360\end{array}$ \\
\hline $\begin{array}{l}1983 \\
1983 \\
1983 \\
1983 \\
1983\end{array}$ & $\begin{array}{l}\text { NOV } \\
\text { DEC } \\
\text { DEC } \\
\text { DEC } \\
\text { DEC }\end{array}$ & $\begin{array}{l}21 \\
01 \\
03 \\
05 \\
05\end{array}$ & $\begin{array}{l}19 \\
07 \\
03 \\
02 \\
11\end{array}$ & $\begin{array}{l}11 \\
52 \\
10 \\
13 \\
51\end{array}$ & $\begin{array}{l}35.7 \\
58.5 \\
16.0 \\
27.1 \\
02.4\end{array}$ & $\begin{array}{l}44.027 \mathrm{~N} \\
44.285 \mathrm{~N} \\
44.480 \mathrm{~N} \\
44.302 \mathrm{~N} \\
44.362 \mathrm{~N}\end{array}$ & $\begin{array}{l}113.952 W . \\
114.133 W . \\
114.103 W . \\
113.816 W . \\
114.120 W .\end{array}$ & $\begin{array}{l}010 \\
010 \\
010 \\
010 \\
010\end{array}$ & $\begin{array}{l}c \\
C \\
C \\
C \\
C\end{array}$ & $\begin{array}{l}360 \\
360 \\
360 \\
360 \\
360\end{array}$ & $\begin{array}{l}\cdots \\
\cdots \\
\cdots \\
\cdots\end{array}$ & $\begin{array}{l}\cdots \\
\cdots \\
\cdots \\
\cdots\end{array}$ & $\begin{array}{l}\text { 3. } 6 \mathrm{ML} \\
3.5 \mathrm{ML} \\
3.3 \mathrm{ML} \\
3.5 \mathrm{ML} \\
3.2 \mathrm{ML}\end{array}$ & $\begin{array}{l}\text { GS } \\
\text { GS } \\
\text { GS } \\
\text { GS } \\
\text { GS }\end{array}$ & $\begin{array}{l}\cdots \\
\cdots \\
\cdots \\
\cdots\end{array}$ & $\begin{array}{l}\cdots \\
\cdots \\
\cdots \\
\cdots \\
\cdots\end{array}$ & $\begin{array}{l}\cdots \\
\cdots \\
\cdots \\
\cdots \\
\cdots\end{array}$ & $\begin{array}{l}\cdots \\
\cdots \\
\cdots \\
\cdots\end{array}$ \\
\hline $\begin{array}{l}1983 \\
1983 \\
1983 \\
1983 \\
1983\end{array}$ & $\begin{array}{l}\text { DEC } \\
\text { DEC } \\
\text { DEC } \\
\text { DEC } \\
\text { DEC }\end{array}$ & $\begin{array}{l}08 \\
10 \\
11 \\
11 \\
12\end{array}$ & $\begin{array}{l}04 \\
01 \\
07 \\
19 \\
04\end{array}$ & $\begin{array}{l}24 \\
35 \\
40 \\
58 \\
55\end{array}$ & $\begin{array}{l}50.0 \\
01.0 \\
45.7 \\
18.2 \\
36.5\end{array}$ & $\begin{array}{l}44.179 \mathrm{~N} \\
44.256 \mathrm{~N} \\
42.352 \mathrm{~N} \\
44.286 \mathrm{~N} \\
44.413 \mathrm{~N}\end{array}$ & $\begin{array}{l}113.907 W . \\
114.156 W . \\
111.569 W . \\
114.160 W . \\
114.086 W .\end{array}$ & $\begin{array}{l}010 \\
010 \\
006 \\
010 \\
007\end{array}$ & $\begin{array}{l}D \\
C \\
B \\
C \\
A\end{array}$ & $\begin{array}{l}360 \\
360 \\
360 \\
360 \\
354\end{array}$ & $\begin{array}{c}\cdots \\
\cdots \\
\cdots \\
4.5\end{array}$ & $\begin{array}{l}\cdots \\
\cdots \\
\cdots \\
\cdots\end{array}$ & $\begin{array}{l}\text { 3. } 4 M L \\
\text { 3. } 8 M L \\
\text { 3. } 6 \mathrm{ML} \\
\text { 4. } 8 \mathrm{ML} \\
\text { 4. } 4 \mathrm{ML}\end{array}$ & $\begin{array}{l}\text { GS } \\
\text { GS } \\
\text { UU } \\
\text { GS } \\
\text { MSO }\end{array}$ & $\begin{array}{l}\cdots \\
\cdots \\
\cdots \\
\cdots\end{array}$ & $\begin{array}{l}\cdots \\
\cdots \\
\cdots \\
\cdots \\
\cdots\end{array}$ & $\begin{array}{l}\cdots \\
\dot{I} i \mathrm{I} \\
\cdots\end{array}$ & $\begin{array}{c}\ldots \\
360 \\
3 \dot{60}\end{array}$ \\
\hline $\begin{array}{l}1983 \\
1983 \\
1983 \\
1983 \\
1983\end{array}$ & $\begin{array}{l}\text { DEC } \\
\text { DEC } \\
\text { DEC } \\
\text { DEC } \\
\text { DEC }\end{array}$ & $\begin{array}{l}12 \\
13 \\
13 \\
15 \\
17\end{array}$ & $\begin{array}{l}05 \\
14 \\
17 \\
06 \\
18\end{array}$ & $\begin{array}{l}11 \\
55 \\
13 \\
13 \\
37\end{array}$ & $\begin{array}{l}27.8 \\
26.9 \\
38.6 \\
34.8 \\
20.7\end{array}$ & $\begin{array}{l}44.423 N \\
44.226 \mathrm{~N} \\
44.244 \mathrm{~N} \\
44.365 \mathrm{~N} \\
44.237 \mathrm{~N}\end{array}$ & $\begin{array}{l}114.114 W . \\
114.177 W . \\
114.074 W . \\
114.138 W . \\
114.085 W .\end{array}$ & $\begin{array}{l}010 \\
010 \\
010 \\
010 \\
010\end{array}$ & $\begin{array}{l}c \\
c \\
C \\
c \\
C\end{array}$ & $\begin{array}{l}360 \\
360 \\
360 \\
360 \\
360\end{array}$ & $\begin{array}{l}\cdots \\
\cdots \\
\cdots \\
\cdots\end{array}$ & $\begin{array}{l}\cdots \\
\cdots \\
\cdots \\
\cdots\end{array}$ & $\begin{array}{l}\text { 3. } 2 \mathrm{ML} \\
\text { 3. } 2 \mathrm{ML} \\
\text { 3. } 6 \mathrm{ML} \\
\text { 4. } 1 \mathrm{ML} \\
\text { 3. } 7 \mathrm{ML}\end{array}$ & $\begin{array}{l}\text { GS } \\
\text { GS } \\
\text { GS } \\
\text { GS } \\
\text { GS }\end{array}$ & $\begin{array}{l}\cdots \\
\cdots \\
\cdots \\
\cdots\end{array}$ & $\begin{array}{l}\cdots \\
\cdots \\
\cdots \\
\cdots \\
\cdots\end{array}$ & $\begin{array}{l}\cdots \\
\cdots \\
\text { IV } \\
\text { F }\end{array}$ & $\begin{array}{l}\cdots \\
360 \\
360 \\
360\end{array}$ \\
\hline $\begin{array}{l}1983 \\
1983 \\
1983 \\
1983 \\
1983\end{array}$ & $\begin{array}{l}\text { DEC } \\
\text { DEC } \\
\text { DEC } \\
\text { DEC } \\
\text { DEC }\end{array}$ & $\begin{array}{l}19 \\
19 \\
20 \\
20 \\
21\end{array}$ & $\begin{array}{l}11 \\
17 \\
03 \\
17 \\
02\end{array}$ & $\begin{array}{l}31 \\
27 \\
40 \\
36 \\
54\end{array}$ & $\begin{array}{l}34.4 \\
55.9 \\
42.4 \\
22.0 \\
17.0\end{array}$ & $\begin{array}{l}44.305 \mathrm{~N} \\
44.397 \mathrm{~N} \\
44.215 \mathrm{~N} \\
44.487 \mathrm{~N} \\
44.125 \mathrm{~N}\end{array}$ & $\begin{array}{l}114.008 W . \\
114.179 w . \\
114.059 w . \\
111.146 w . \\
114.033 w .\end{array}$ & $\begin{array}{l}010 \\
010 \\
010 \\
010 \\
010\end{array}$ & $\begin{array}{l}C \\
D \\
D \\
C \\
C\end{array}$ & $\begin{array}{l}360 \\
360 \\
360 \\
360 \\
360\end{array}$ & $\begin{array}{l}\cdots \\
\cdots \\
\cdots \\
\cdots\end{array}$ & $\begin{array}{l}\cdots \\
\cdots \\
\cdots \\
\cdots\end{array}$ & $\begin{array}{l}\text { 3. } 5 \mathrm{ML} \\
\text { 3. } 6 \mathrm{ML} \\
3.1 \mathrm{ML} \\
3.0 \mathrm{ML} \\
\text { 3. } 8 \mathrm{ML}\end{array}$ & $\begin{array}{l}\text { GS } \\
\text { GS } \\
\text { GS } \\
\text { MMT } \\
\text { GS }\end{array}$ & $\begin{array}{l}\cdots \\
\cdots \\
\cdots \\
\cdots\end{array}$ & $\begin{array}{l}\cdots \\
\cdots \\
\cdots \\
\cdots \\
\cdots\end{array}$ & $\begin{array}{l}\cdots \\
\cdots \\
\cdots \\
\text { III }\end{array}$ & $\begin{array}{c}\cdots \\
\cdots \\
360\end{array}$ \\
\hline $\begin{array}{l}1983 \\
1983 \\
1983 \\
1983 \\
1983\end{array}$ & $\begin{array}{l}\text { DEC } \\
\text { DEC } \\
\text { DEC } \\
\text { DEC } \\
\text { DEC }\end{array}$ & $\begin{array}{l}21 \\
25 \\
25 \\
27 \\
27\end{array}$ & $\begin{array}{l}06 \\
09 \\
12 \\
12 \\
20\end{array}$ & $\begin{array}{l}33 \\
49 \\
23 \\
21 \\
19\end{array}$ & $\begin{array}{l}54.9 \\
01.5 \\
56.3 \\
29.3 \\
17.7\end{array}$ & $\begin{array}{l}44.365 \mathrm{~N} \\
44.143 \mathrm{~N} \\
44.342 \mathrm{~N} \\
44.255 \mathrm{~N} \\
44.223 \mathrm{~N}\end{array}$ & $\begin{array}{l}113.984 W . \\
113.924 W . \\
114.093 W . \\
114.074 W . \\
114.081 W .\end{array}$ & $\begin{array}{l}010 \\
010 \\
010 \\
009 \\
010\end{array}$ & $\begin{array}{l}C \\
C \\
C \\
A \\
C\end{array}$ & $\begin{array}{l}360 \\
360 \\
360 \\
360 \\
360\end{array}$ & $\begin{array}{c}\ldots \\
\ldots \\
4 . \\
\ldots\end{array}$ & $\begin{array}{l}\cdots \\
\cdots \\
\cdots \\
\cdots\end{array}$ & $\begin{array}{l}\text { 3. } 3 \mathrm{ML} \\
3.6 \mathrm{ML} \\
3.4 \mathrm{ML} \\
.0 \mathrm{ML}\end{array}$ & $\begin{array}{l}\text { GS } \\
\text { GS } \\
\text { GS } \\
\ddot{G S}\end{array}$ & $\begin{array}{l}\cdots \\
\cdots \\
\cdots \\
\cdots\end{array}$ & $\begin{array}{l}\cdots \\
\cdots \\
\cdots \\
\cdots \\
\cdots\end{array}$ & $\begin{array}{l}\cdots \\
\cdots \\
\text { IV } \\
\cdots\end{array}$ & $\begin{array}{c}\cdots \\
36 \\
\cdots\end{array}$ \\
\hline $\begin{array}{l}1983 \\
1983 \\
1983 \\
1983 \\
1984\end{array}$ & $\begin{array}{l}\text { DEC } \\
\text { DEC } \\
\text { DEC } \\
\text { DEC } \\
\text { JAN }\end{array}$ & $\begin{array}{l}28 \\
29 \\
29 \\
31 \\
01\end{array}$ & $\begin{array}{l}08 \\
03 \\
16 \\
12 \\
12\end{array}$ & $\begin{array}{l}16 \\
44 \\
05 \\
10 \\
27\end{array}$ & $\begin{array}{l}53.6 \\
39.8 \\
24.4 \\
13.2 \\
14.4\end{array}$ & $\begin{array}{l}44.281 \mathrm{~N} \\
44.289 \mathrm{~N} \\
44.419 \mathrm{~N} \\
44.264 \mathrm{~N} \\
44.261 \mathrm{~N}\end{array}$ & $\begin{array}{l}114.089 W . \\
114.047 W . \\
114.059 W . \\
114.113 W . \\
113.985 W .\end{array}$ & $\begin{array}{l}011 \\
010 \\
010 \\
010 \\
010\end{array}$ & $\begin{array}{l}B \\
C \\
C \\
C \\
C\end{array}$ & $\begin{array}{l}360 \\
360 \\
360 \\
360 \\
370\end{array}$ & $\begin{array}{l}\cdots \\
\cdots \\
\cdots \\
\cdots\end{array}$ & $\begin{array}{l}\cdots \\
\cdots \\
\cdots \\
\cdots\end{array}$ & $\begin{array}{l}\text { 4. } \mathrm{OML} \\
\text { 3. } 3 \mathrm{ML} \\
\text { 3. } 8 \mathrm{ML} \\
\text { 3. } 6 \mathrm{ML} \\
\text { 3. } 4 \mathrm{ML}\end{array}$ & $\begin{array}{l}\text { GS } \\
\text { GS } \\
\text { GS } \\
\text { GS } \\
\text { GS }\end{array}$ & $\begin{array}{l}\cdots \\
\cdots \\
\cdots \\
\cdots\end{array}$ & $\begin{array}{l}\cdots \\
\cdots \\
\cdots \\
\cdots \\
\cdots\end{array}$ & $\begin{array}{l}\text { IV } \\
\dot{\text { III }} \\
\ldots \\
\cdots\end{array}$ & $\begin{array}{c}360 \\
360 \\
\ldots \\
\ldots\end{array}$ \\
\hline $\begin{array}{l}1984 \\
1984 \\
1984 \\
1984 \\
1984\end{array}$ & $\begin{array}{l}\text { JAN } \\
\text { JAN } \\
\text { JAN } \\
\text { JAN } \\
\text { JAN }\end{array}$ & $\begin{array}{l}03 \\
03 \\
05 \\
11 \\
15\end{array}$ & $\begin{array}{l}09 \\
09 \\
02 \\
07 \\
00\end{array}$ & $\begin{array}{l}30 \\
40 \\
02 \\
35 \\
05\end{array}$ & $\begin{array}{l}08.4 \\
32.9 \\
51.3 \\
44.1 \\
34.1\end{array}$ & $\begin{array}{l}44.305 \mathrm{~N} \\
44.281 \mathrm{~N} \\
44.395 \mathrm{~N} \\
44.287 \mathrm{~N} \\
44.264 \mathrm{~N}\end{array}$ & $\begin{array}{l}114.113 W . \\
114.107 W . \\
114.160 W . \\
114.093 W . \\
114.157 W .\end{array}$ & $\begin{array}{l}010 \\
010 \\
010 \\
010 \\
010\end{array}$ & $\begin{array}{l}C \\
C \\
D \\
B \\
C\end{array}$ & $\begin{array}{l}370 \\
370 \\
370 \\
370 \\
370\end{array}$ & $\begin{array}{l}\cdots \\
\cdots \\
\cdots \\
\cdots\end{array}$ & $\begin{array}{l}\cdots \\
\cdots \\
\cdots \\
\cdots\end{array}$ & $\begin{array}{l}\text { 3. } \mathrm{OML} \\
\text { 3. } 3 \mathrm{ML} \\
\text { 3. } 2 \mathrm{ML} \\
\text { 4. } \mathrm{OML} \\
\text { 3. } 2 \mathrm{ML}\end{array}$ & $\begin{array}{l}\text { GS } \\
\text { GS } \\
\text { GS } \\
\text { GS } \\
\text { GS }\end{array}$ & $\begin{array}{l}\cdots \\
\cdots \\
\cdots \\
\cdots\end{array}$ & $\begin{array}{l}\cdots \\
\cdots \\
\cdots \\
\cdots \\
\cdots\end{array}$ & $\begin{array}{l}\cdots \\
\cdots \\
\cdots \\
\cdots \\
\cdots\end{array}$ & $\begin{array}{l}\cdots \\
\cdots \\
\cdots \\
\cdots\end{array}$ \\
\hline $\begin{array}{l}1984 \\
1984 \\
1984 \\
1984 \\
1984\end{array}$ & $\begin{array}{l}\text { JAN } \\
\text { JAN } \\
\text { JAN } \\
\text { JAN } \\
\text { JAN }\end{array}$ & $\begin{array}{l}24 \\
25 \\
29 \\
29 \\
29\end{array}$ & $\begin{array}{l}21 \\
19 \\
03 \\
07 \\
12\end{array}$ & $\begin{array}{l}07 \\
36 \\
22 \\
03 \\
05\end{array}$ & $\begin{array}{l}57.5 \\
12.0 \\
25.4 \\
46.1 \\
06.9\end{array}$ & $\begin{array}{l}44.047 \mathrm{~N} \\
47.500 \mathrm{~N} \\
44.721 \mathrm{~N} \\
44.573 \mathrm{~N} \\
44.098 \mathrm{~N}\end{array}$ & $\begin{array}{l}114.442 W \\
116.000 W . \\
114.442 W . \\
114.264 W . \\
113.905 W .\end{array}$ & $\begin{array}{l}010 \\
001 \\
010 \\
010 \\
010\end{array}$ & $\begin{array}{l}B \\
B \\
C \\
C \\
C\end{array}$ & $\begin{array}{l}370 \\
370 \\
370 \\
370 \\
370\end{array}$ & $\begin{array}{c}4.5 \\
\ldots \\
\ldots \\
\ldots \\
\ldots\end{array}$ & $\begin{array}{l}\cdots \\
\cdots \\
\cdots \\
\cdots\end{array}$ & $\begin{array}{l}\text { 4. } 6 \mathrm{ML} \\
\text { 2. } 3 \mathrm{ML} \\
\text { 3. } \mathrm{OML} \\
\text { 3. } 1 \mathrm{ML} \\
\text { 3. } 4 \mathrm{ML}\end{array}$ & $\begin{array}{l}\text { MMT } \\
\text { GS } \\
\text { GS } \\
\text { GS } \\
\text { GS }\end{array}$ & $\begin{array}{l}\cdots \\
\cdots \\
\cdots \\
\cdots\end{array}$ & $\begin{array}{l}\cdots \\
\cdots \\
\cdots \\
\cdots\end{array}$ & $\begin{array}{r}\text { IV } \\
\text { F } \\
\ldots \\
\cdots \\
\cdots\end{array}$ & $\begin{array}{r}370 \\
370 \\
\ldots \\
\ldots \\
\ldots\end{array}$ \\
\hline $\begin{array}{l}1984 \\
1984 \\
1984 \\
1984 \\
1984\end{array}$ & $\begin{array}{l}\text { JAN } \\
\text { JAN } \\
\text { JAN } \\
\text { JAN } \\
\text { JAN }\end{array}$ & $\begin{array}{l}31 \\
31 \\
31 \\
31 \\
31\end{array}$ & $\begin{array}{l}03 \\
08 \\
09 \\
11 \\
16\end{array}$ & $\begin{array}{l}24 \\
25 \\
57 \\
45 \\
50\end{array}$ & $\begin{array}{l}46.5 \\
35.7 \\
44.0 \\
25.6 \\
07.5\end{array}$ & $\begin{array}{l}44.768 \mathrm{~N} \\
44.205 \mathrm{~N} \\
47.414 \mathrm{~N} \\
44.775 \mathrm{~N} \\
44.735 \mathrm{~N}\end{array}$ & $\begin{array}{l}114.488 W . \\
114.032 W . \\
115.944 W . \\
114.460 W . \\
114.470 W .\end{array}$ & $\begin{array}{l}010 \\
010 \\
000 \\
010 \\
010\end{array}$ & $\begin{array}{l}C \\
C \\
C \\
D \\
C\end{array}$ & $\begin{array}{l}370 \\
370 \\
370 \\
370 \\
370\end{array}$ & $\begin{array}{c}\cdots \\
\cdots \\
3.7 \\
\ldots\end{array}$ & $\begin{array}{l}\cdots \\
\cdots \\
\cdots \\
\cdots\end{array}$ & $\begin{array}{l}\text { 3. } 2 \mathrm{ML} \\
\text { 3. } 2 \mathrm{ML} \\
\text { 3. } 1 \mathrm{MD} \\
\text { 3. } 2 \mathrm{ML} \\
\text { 3. } \mathrm{OML}\end{array}$ & $\begin{array}{l}\text { GS } \\
\text { GS } \\
\text { MMT } \\
\text { GS } \\
\text { GS }\end{array}$ & $\begin{array}{l}\cdots \\
\cdots \\
\cdots \\
\cdots\end{array}$ & $\begin{array}{l}\cdots \\
\cdots \\
\cdots \\
\cdots \\
\cdots\end{array}$ & $\begin{array}{l}\cdots \\
\cdots \\
\cdots \\
\cdots \\
\cdots\end{array}$ & $\begin{array}{l}\cdots \\
\cdots \\
\cdots \\
\cdots\end{array}$ \\
\hline $\begin{array}{l}1984 \\
1984 \\
1984 \\
1984 \\
1984\end{array}$ & $\begin{array}{l}\text { FEB } \\
\text { FEB } \\
\text { FEB } \\
\text { FEB } \\
\text { FEB }\end{array}$ & $\begin{array}{l}01 \\
01 \\
01 \\
02 \\
03\end{array}$ & $\begin{array}{l}13 \\
16 \\
22 \\
16 \\
05\end{array}$ & $\begin{array}{l}18 \\
12 \\
49 \\
36 \\
05\end{array}$ & $\begin{array}{l}08.9 \\
35.4 \\
16.6 \\
33.2 \\
25.1\end{array}$ & $\begin{array}{l}44.703 \mathrm{~N} \\
44.746 \mathrm{~N} \\
44.548 \mathrm{~N} \\
44.672 \mathrm{~N} \\
44.726 \mathrm{~N}\end{array}$ & $\begin{array}{l}114.472 W . \\
114.370 W . \\
114.214 W . \\
114.506 W . \\
114.432 W .\end{array}$ & $\begin{array}{l}010 \\
010 \\
010 \\
010 \\
010\end{array}$ & $\begin{array}{l}c \\
C \\
C \\
C \\
C\end{array}$ & $\begin{array}{l}370 \\
370 \\
370 \\
370 \\
370\end{array}$ & $\begin{array}{l}\cdots \\
\cdots \\
\cdots \\
\cdots\end{array}$ & $\begin{array}{l}\cdots \\
\cdots \\
\cdots \\
\cdots\end{array}$ & $\begin{array}{l}3.4 \mathrm{ML} \\
3.1 \mathrm{ML} \\
3.2 \mathrm{ML} \\
3.4 \mathrm{ML} \\
3.2 \mathrm{ML}\end{array}$ & $\begin{array}{l}\text { GS } \\
\text { GS } \\
\text { GS } \\
\text { GS } \\
\text { GS }\end{array}$ & $\begin{array}{l}\cdots \\
\cdots \\
\cdots \\
\cdots\end{array}$ & $\begin{array}{l}\cdots \\
\cdots \\
\cdots \\
\cdots\end{array}$ & $\begin{array}{l}\cdots \\
\cdots \\
\cdots \\
\cdots \\
\cdots\end{array}$ & $\begin{array}{l}\cdots \\
\cdots \\
\cdots \\
\cdots\end{array}$ \\
\hline
\end{tabular}




\begin{tabular}{|c|c|c|c|c|c|c|c|c|c|c|c|c|c|c|c|c|c|c|}
\hline $\begin{array}{l}1984 \\
1984 \\
1984 \\
1984 \\
1984\end{array}$ & $\begin{array}{l}\text { FEB } \\
\text { FEB } \\
\text { FEB } \\
\text { FEB } \\
\text { FEB }\end{array}$ & $\begin{array}{l}05 \\
05 \\
05 \\
06 \\
07\end{array}$ & $\begin{array}{l}03 \\
03 \\
14 \\
23 \\
19\end{array}$ & $\begin{array}{l}42 \\
44 \\
02 \\
04 \\
00\end{array}$ & $\begin{array}{l}29.6 \\
15.8 \\
54.7 \\
32.1 \\
40.1\end{array}$ & $\begin{array}{l}44.276 \mathrm{~N} \\
44.275 \mathrm{~N} \\
44.093 \mathrm{~N} \\
44.540 \mathrm{~N} \\
44.438 \mathrm{~N}\end{array}$ & $\begin{array}{l}114.124 W . \\
114.127 W . \\
114.196 w . \\
114.266 W . \\
114.137 w .\end{array}$ & $\begin{array}{l}010 \\
010 \\
010 \\
010 \\
010\end{array}$ & $\begin{array}{l}C \\
C \\
C \\
C \\
C\end{array}$ & $\begin{array}{l}370 \\
370 \\
370 \\
370 \\
370\end{array}$ & $\begin{array}{l}\cdots \\
\cdots \\
\cdots \\
\cdots\end{array}$ & $\begin{array}{l}\cdots \\
\cdots \\
\therefore \\
\cdots\end{array}$ & $\begin{array}{l}3.1 \mathrm{ML} \\
3.9 \mathrm{ML} \\
3.2 \mathrm{ML} \\
3.1 \mathrm{ML} \\
3.2 \mathrm{ML}\end{array}$ & $\begin{array}{l}\text { GS } \\
\text { GS } \\
\text { GS } \\
\text { GS } \\
\text { GS }\end{array}$ & $\begin{array}{l}\cdots \\
\cdots \\
\cdots \\
\cdots\end{array}$ & $\begin{array}{l}\cdots \\
\cdots \\
\cdots \\
\cdots \\
\cdots\end{array}$ & $\begin{array}{l}\cdots \dot{\boldsymbol{F}} \\
\cdots \\
\cdots \\
\cdots\end{array}$ & $\begin{array}{r}370 \\
\cdots \\
\cdots\end{array}$ \\
\hline $\begin{array}{l}1984 \\
1984 \\
1984 \\
1984 \\
1984\end{array}$ & $\begin{array}{l}\text { FEB } \\
\text { FEB } \\
\text { FEB } \\
\text { FEB } \\
\text { FEB }\end{array}$ & $\begin{array}{l}08 \\
08 \\
10 \\
15 \\
19\end{array}$ & $\begin{array}{l}15 \\
18 \\
16 \\
18 \\
21\end{array}$ & $\begin{array}{l}02 \\
17 \\
06 \\
34 \\
48\end{array}$ & $\begin{array}{l}32.4 \\
43.9 \\
15.7 \\
36.6 \\
50.5\end{array}$ & $\begin{array}{l}44.702 \mathrm{~N} . \\
44.431 \mathrm{~N} . \\
44.333 \mathrm{~N} . \\
44.294 \mathrm{~N} \\
44.333 \mathrm{~N} .\end{array}$ & $\begin{array}{l}114.437 w . \\
114.168 w . \\
114.107 w . \\
114.000 w . \\
114.105 w .\end{array}$ & $\begin{array}{l}010 \\
010 \\
010 \\
010 \\
010\end{array}$ & $\begin{array}{l}C \\
C \\
D \\
D \\
C\end{array}$ & $\begin{array}{l}370 \\
370 \\
370 \\
370 \\
370\end{array}$ & $\begin{array}{l}\cdots \\
\cdots \\
\cdots \\
\cdots\end{array}$ & $\begin{array}{l}\cdots \\
\cdots \\
\cdots \\
\cdots\end{array}$ & $\begin{array}{l}\text { 3. } 0 \mathrm{ML} \\
3.4 \mathrm{ML} \\
3.1 \mathrm{ML} \\
3.2 \mathrm{ML} \\
3.0 \mathrm{ML}\end{array}$ & $\begin{array}{l}\text { GS } \\
\text { GS } \\
\text { GS } \\
\text { GS } \\
\text { GS }\end{array}$ & $\begin{array}{l}\cdots \\
\cdots \\
\cdots \\
\cdots\end{array}$ & $\begin{array}{l}\cdots \\
\cdots \\
\cdots \\
\cdots \\
\cdots\end{array}$ & $\begin{array}{l}\cdots \\
\cdots \\
\cdots \\
\cdots \\
\cdots\end{array}$ & $\begin{array}{l}\cdots \\
\cdots \\
\cdots \\
\cdots\end{array}$ \\
\hline $\begin{array}{l}1984 \\
1984 \\
1984 \\
1984 \\
1984\end{array}$ & $\begin{array}{l}\text { FEB } \\
\text { FEB } \\
\text { FEB } \\
\text { FEB } \\
\text { MAR }\end{array}$ & $\begin{array}{l}20 \\
20 \\
25 \\
27 \\
02\end{array}$ & $\begin{array}{l}02 \\
16 \\
11 \\
11 \\
00\end{array}$ & $\begin{array}{l}58 \\
17 \\
11 \\
52 \\
29\end{array}$ & $\begin{array}{l}32.9 \\
25.5 \\
21.0 \\
11.2 \\
45.1\end{array}$ & $\begin{array}{l}44.437 \mathrm{~N} \\
44.432 \mathrm{~N} \\
44.197 \mathrm{~N} \\
44.222 \mathrm{~N} \\
44.350 \mathrm{~N}\end{array}$ & $\begin{array}{l}114.089 w . \\
114.194 w . \\
114.093 w . \\
114.003 w . \\
114.186 w .\end{array}$ & $\begin{array}{l}010 \\
010 \\
010 \\
010 \\
010\end{array}$ & $\begin{array}{l}\mathrm{C} \\
\mathrm{C} \\
\mathrm{C} \\
\mathrm{C} \\
\mathrm{B}\end{array}$ & $\begin{array}{l}370 \\
370 \\
370 \\
370 \\
370\end{array}$ & $\begin{array}{c}\cdots \\
\cdots \\
\cdots \\
4.5\end{array}$ & $\begin{array}{l}\cdots \\
\cdots \\
\cdots \\
\cdots\end{array}$ & $\begin{array}{l}\text { 3. } 7 \mathrm{ML} \\
\text { 3. } 6 \mathrm{ML} \\
\text { 3. } 4 \mathrm{ML} \\
\text { 3. } \mathrm{MML} \\
\text { 4. } 3 \mathrm{ML}\end{array}$ & $\begin{array}{l}\text { GS } \\
\text { GS } \\
\text { GS } \\
\text { GS } \\
\text { GS }\end{array}$ & $\begin{array}{l}\cdots \\
\cdots \\
\cdots \\
\cdots\end{array}$ & $\begin{array}{l}\cdots \\
\cdots \\
\cdots \\
\cdots \\
\cdots\end{array}$ & $\begin{array}{l}\cdots \\
\cdots \\
\cdots \\
\cdots \\
\text { IV }\end{array}$ & $\begin{array}{c}\cdots \\
\cdots \\
3 \\
3 \dot{7}\end{array}$ \\
\hline $\begin{array}{l}1984 \\
1984 \\
1984 \\
1984 \\
1984\end{array}$ & $\begin{array}{l}\text { MAR } \\
\text { MAR } \\
\text { MAR } \\
\text { MAR } \\
\text { MAR }\end{array}$ & $\begin{array}{l}02 \\
06 \\
06 \\
07 \\
08\end{array}$ & $\begin{array}{l}00 \\
15 \\
19 \\
18 \\
11\end{array}$ & $\begin{array}{l}52 \\
22 \\
51 \\
32 \\
19\end{array}$ & $\begin{array}{l}25.3 \\
30.5 \\
35.3 \\
92.9 \\
43.6\end{array}$ & $\begin{array}{l}44.320 \mathrm{~N} \\
44.197 \mathrm{~N} . \\
44.349 \mathrm{~N} \\
44.335 \mathrm{~N} \\
44.467 \mathrm{~N}\end{array}$ & $\begin{array}{l}114.173 w . \\
114.457 w . \\
114.586 w . \\
114.387 w . \\
112.643 w .\end{array}$ & $\begin{array}{l}010 \\
010 \\
010 \\
010 \\
005\end{array}$ & $\begin{array}{l}C \\
C \\
C \\
C \\
C\end{array}$ & $\begin{array}{l}370 \\
370 \\
370 \\
370 \\
370\end{array}$ & $\begin{array}{l}\cdots \\
\cdots \\
\cdots \\
\cdots\end{array}$ & $\begin{array}{l}\cdots \\
\cdots \\
\cdots \\
\cdots\end{array}$ & $\begin{array}{l}\text { 3. OML } \\
\text { 3. } 3 M L \\
\text { 3. OML } \\
\text { 3. OML } \\
\text { 3. } 1 \mathrm{ML}\end{array}$ & $\begin{array}{l}\text { GS } \\
\text { GS } \\
\text { GS } \\
\text { GS } \\
\text { MMT }\end{array}$ & $\begin{array}{l}\cdots \\
\cdots \\
\cdots \\
\cdots\end{array}$ & $\begin{array}{l}\cdots \\
\ldots \\
\cdots \\
\cdots \\
\cdots\end{array}$ & $\begin{array}{l}\cdots \\
\ldots \\
\cdots \\
\cdots \\
\cdots\end{array}$ & $\begin{array}{l}\cdots \\
\cdots \\
\cdots \\
\cdots\end{array}$ \\
\hline $\begin{array}{l}1984 \\
1984 \\
1984 \\
1984 \\
1984\end{array}$ & $\begin{array}{l}\text { MAR } \\
\text { MAR } \\
\text { MAR } \\
\text { MAR } \\
\text { MAR }\end{array}$ & $\begin{array}{l}09 \\
09 \\
09 \\
09 \\
10\end{array}$ & $\begin{array}{l}01 \\
01 \\
10 \\
23 \\
04\end{array}$ & $\begin{array}{l}16 \\
55 \\
49 \\
49 \\
53\end{array}$ & $\begin{array}{l}14.2 \\
34.0 \\
37.2 \\
12.4 \\
46.9\end{array}$ & $\begin{array}{l}44.330 \mathrm{~N} \\
44.302 \mathrm{~N} \\
43.971 \mathrm{~N} \\
44.151 \mathrm{~N} . \\
44.437 \mathrm{~N}\end{array}$ & $\begin{array}{l}114.009 w . \\
114.102 w . \\
113.730 w . \\
113.977 w . \\
112.638 w .\end{array}$ & $\begin{array}{l}005 \\
010 \\
010 \\
010 \\
005\end{array}$ & $\begin{array}{l}c \\
C \\
C \\
C \\
C\end{array}$ & $\begin{array}{l}370 \\
370 \\
370 \\
370 \\
370\end{array}$ & $\begin{array}{l}\cdots \\
\cdots \\
\cdots \\
\cdots\end{array}$ & $\begin{array}{l}\cdots \\
\cdots \\
\cdots \\
\cdots\end{array}$ & $\begin{array}{l}\text { 3. } 3 M L \\
3.3 M L \\
3.3 M L \\
3.4 M L \\
3.3 M L\end{array}$ & $\begin{array}{l}\text { GS } \\
\text { GS } \\
\text { GS } \\
\text { GS } \\
\text { GS }\end{array}$ & $\begin{array}{l}\cdots \\
\cdots \\
\cdots \\
\cdots\end{array}$ & $\begin{array}{l}\cdots \\
\cdots \\
\cdots \\
\cdots \\
\cdots\end{array}$ & $\begin{array}{l}\cdots \\
\cdots \\
\cdots \\
\cdots \\
\cdots\end{array}$ & $\begin{array}{l}\cdots \\
\cdots \\
\cdots \\
\cdots\end{array}$ \\
\hline $\begin{array}{l}1984 \\
1984 \\
1984 \\
1984 \\
1984\end{array}$ & $\begin{array}{l}\text { MAR } \\
\text { MAR } \\
\text { MAR } \\
\text { MAR } \\
\text { MAR }\end{array}$ & $\begin{array}{l}12 \\
16 \\
17 \\
21 \\
23\end{array}$ & $\begin{array}{l}03 \\
14 \\
02 \\
21 \\
01\end{array}$ & $\begin{array}{l}21 \\
35 \\
10 \\
43 \\
39\end{array}$ & $\begin{array}{l}56.7 \\
38.9 \\
20.4 \\
36.5 \\
37.3\end{array}$ & $\begin{array}{l}44.347 \mathrm{~N} \\
44.309 \mathrm{~N} . \\
44.266 \mathrm{~N} \\
44.730 \mathrm{~N} \\
44.696 \mathrm{~N}\end{array}$ & $\begin{array}{l}114.317 w . \\
114.544 W . \\
114.147 w . \\
114.438 w . \\
114.531 w .\end{array}$ & $\begin{array}{l}010 \\
010 \\
010 \\
010 \\
010\end{array}$ & $\begin{array}{l}C \\
C \\
C \\
C \\
C\end{array}$ & $\begin{array}{l}370 \\
370 \\
370 \\
370 \\
370\end{array}$ & $\begin{array}{l}\cdots \\
\cdots \\
\cdots \\
\cdots\end{array}$ & $\begin{array}{l}\cdots \\
\cdots \\
\cdots \\
\cdots\end{array}$ & $\begin{array}{l}\text { 3. } 2 \mathrm{ML} \\
\text { 3. } 2 \mathrm{ML} \\
\text { 3. } 2 \mathrm{ML} \\
\text { 3. } 8 \mathrm{ML} \\
\text { 3. } 2 \mathrm{ML}\end{array}$ & $\begin{array}{l}\text { GS } \\
\text { MMT } \\
\text { GS } \\
\text { GS } \\
\text { GS }\end{array}$ & $\begin{array}{l}\cdots \\
\cdots \\
\cdots \\
\cdots\end{array}$ & $\begin{array}{l}\cdots \\
\cdots \\
\cdots \\
\cdots \\
\cdots\end{array}$ & $\begin{array}{l}\cdots \\
\cdots \\
\cdots \\
\cdots \\
\cdots\end{array}$ & $\begin{array}{l}\cdots \\
\cdots \\
\cdots \\
\cdots\end{array}$ \\
\hline $\begin{array}{l}1984 \\
1984 \\
1984 \\
1984 \\
1984\end{array}$ & $\begin{array}{l}\text { MAR } \\
\text { MAR } \\
\text { MAR } \\
\text { MAR } \\
\text { MAR }\end{array}$ & $\begin{array}{l}24 \\
24 \\
24 \\
24 \\
24\end{array}$ & $\begin{array}{l}00 \\
02 \\
02 \\
03 \\
08\end{array}$ & $\begin{array}{l}07 \\
16 \\
31 \\
03 \\
55\end{array}$ & $\begin{array}{l}47.7 \\
52.8 \\
33.3 \\
15.5 \\
14.5\end{array}$ & $\begin{array}{l}44.740 \mathrm{~N} \\
44.743 \mathrm{~N} \\
44.669 \mathrm{~N} \\
44.722 \mathrm{~N} \\
44.715 \mathrm{~N}\end{array}$ & $\begin{array}{l}114.430 w . \\
114.373 w . \\
114.471 w . \\
114.461 w . \\
114.423 w .\end{array}$ & $\begin{array}{l}010 \\
010 \\
010 \\
010 \\
010\end{array}$ & $\begin{array}{l}B \\
C \\
C \\
B \\
C\end{array}$ & $\begin{array}{l}370 \\
370 \\
370 \\
370 \\
370\end{array}$ & $\begin{array}{c}4.2 \\
\ldots \\
\ldots \\
\ldots \\
\ldots\end{array}$ & $\begin{array}{l}\cdots \\
\cdots \\
\cdots \\
\cdots\end{array}$ & $\begin{array}{l}\text { 4. } 3 M L \\
\text { 3. } O M L \\
\text { 3. } 2 M L \\
\text { 4. } 9 M L \\
\text { 3. } 3 M L\end{array}$ & $\begin{array}{l}\text { MMT } \\
\text { GS } \\
\text { GS } \\
\text { GS } \\
\text { GS }\end{array}$ & $\begin{array}{l}\cdots \\
\cdots \\
\cdots \\
\cdots\end{array}$ & $\begin{array}{l}\cdots \\
\cdots \\
\cdots \\
\cdots \\
\cdots\end{array}$ & $\begin{array}{l}\quad v \\
\cdots \\
\cdots \\
\cdots \\
\cdots\end{array}$ & $\begin{array}{c}370 \\
\ldots \\
\ldots \\
\ldots \\
\ldots\end{array}$ \\
\hline $\begin{array}{l}1984 \\
1984 \\
1984 \\
1984 \\
1984\end{array}$ & $\begin{array}{l}\text { MAR } \\
\text { MAR } \\
\text { MAR } \\
\text { MAR } \\
\text { MAR }\end{array}$ & $\begin{array}{l}24 \\
24 \\
24 \\
24 \\
24\end{array}$ & $\begin{array}{l}09 \\
16 \\
19 \\
20 \\
21\end{array}$ & $\begin{array}{l}53 \\
01 \\
20 \\
02 \\
01\end{array}$ & $\begin{array}{l}42.6 \\
32.6 \\
13.8 \\
06.0 \\
12.3\end{array}$ & $\begin{array}{l}44.693 \mathrm{~N} \\
44.641 \mathrm{~N} . \\
44.721 \mathrm{~N} \\
44.709 \mathrm{~N} \\
44.374 \mathrm{~N}\end{array}$ & $\begin{array}{l}114.534 w . \\
114.670 w . \\
114.443 w . \\
114.452 w . \\
114.086 w .\end{array}$ & $\begin{array}{l}010 \\
010 \\
010 \\
010 \\
010\end{array}$ & $\begin{array}{l}c \\
C \\
C \\
C \\
C\end{array}$ & $\begin{array}{l}370 \\
370 \\
370 \\
370 \\
370\end{array}$ & $\begin{array}{l}\cdots \\
\cdots \\
\cdots \\
\cdots\end{array}$ & $\begin{array}{l}\cdots \\
\cdots \\
\cdots \\
\cdots\end{array}$ & $\begin{array}{l}3.6 \mathrm{ML} \\
3.1 \mathrm{ML} \\
3.3 \mathrm{ML} \\
3.5 \mathrm{ML} \\
3.5 \mathrm{ML}\end{array}$ & $\begin{array}{l}\text { MMT } \\
\text { GS } \\
\text { GS } \\
\text { GS } \\
\text { MMT }\end{array}$ & $\begin{array}{l}\cdots \\
\cdots \\
\cdots \\
\cdots\end{array}$ & $\begin{array}{l}\cdots \\
\cdots \\
\cdots \\
\cdots \\
\cdots\end{array}$ & $\begin{array}{l}\cdots \\
\cdots \\
\cdots \\
\cdots \\
\cdots\end{array}$ & $\begin{array}{l}\cdots \\
\cdots \\
\cdots \\
\cdots\end{array}$ \\
\hline $\begin{array}{l}1984 \\
1984 \\
1984 \\
1984 \\
1984\end{array}$ & $\begin{array}{l}\text { MAR } \\
\text { MAR } \\
\text { MAR } \\
\text { MAR } \\
\text { MAR }\end{array}$ & $\begin{array}{l}24 \\
25 \\
25 \\
29 \\
30\end{array}$ & $\begin{array}{l}21 \\
00 \\
11 \\
11 \\
16\end{array}$ & $\begin{array}{l}03 \\
21 \\
24 \\
43 \\
23\end{array}$ & $\begin{array}{l}16.3 \\
14.5 \\
53.7 \\
55.0 \\
59.2\end{array}$ & $\begin{array}{l}44.728 \mathrm{~N}, \\
44.713 \mathrm{~N}, \\
44.722 \mathrm{~N} . \\
44.708 \mathrm{~N} . \\
44.430 \mathrm{~N} .\end{array}$ & $\begin{array}{l}114.441 w . \\
114.507 w . \\
114.422 w . \\
114.505 w . \\
114.173 w .\end{array}$ & $\begin{array}{l}010 \\
010 \\
010 \\
010 \\
010\end{array}$ & $\begin{array}{l}B \\
C \\
C \\
C \\
C\end{array}$ & $\begin{array}{l}370 \\
370 \\
370 \\
370 \\
370\end{array}$ & $\begin{array}{l}\cdots \\
\cdots \\
\cdots \\
\cdots\end{array}$ & $\begin{array}{l}\cdots \\
\cdots \\
\cdots \\
\cdots\end{array}$ & $\begin{array}{l}\text { 3. } 8 \mathrm{ML} \\
3.2 \mathrm{ML} \\
3.5 \mathrm{ML} \\
3.2 \mathrm{ML} \\
3.5 \mathrm{ML}\end{array}$ & $\begin{array}{l}\text { GS } \\
\text { GS } \\
\text { GS } \\
\text { GS } \\
\text { MMT }\end{array}$ & $\begin{array}{l}\cdots \\
\cdots \\
\cdots \\
\cdots\end{array}$ & $\begin{array}{l}\cdots \\
\cdots \\
\cdots \\
\cdots \\
\cdots\end{array}$ & $\begin{array}{l}\text { II I } \\
\ldots \\
\cdots \\
\cdots \\
\cdots\end{array}$ & $\begin{array}{r}370 \\
\ldots \\
\ldots \\
\ldots \\
\ldots\end{array}$ \\
\hline $\begin{array}{l}1984 \\
1984 \\
1984 \\
1984 \\
1984\end{array}$ & $\begin{array}{l}\text { MAR } \\
\text { MAR } \\
\text { APR } \\
\text { APR } \\
\text { APR }\end{array}$ & $\begin{array}{l}31 \\
31 \\
01 \\
01 \\
01\end{array}$ & $\begin{array}{l}01 \\
23 \\
05 \\
05 \\
09\end{array}$ & $\begin{array}{l}12 \\
07 \\
15 \\
39 \\
13\end{array}$ & $\begin{array}{l}56.4 \\
10.1 \\
18.8 \\
27.8 \\
38.4\end{array}$ & $\begin{array}{l}44.326 \mathrm{~N} . \\
44.728 \mathrm{~N} . \\
44.707 \mathrm{~N} . \\
44.726 \mathrm{~N} . \\
44.739 \mathrm{~N} .\end{array}$ & $\begin{array}{l}114.144 w . \\
114.356 w . \\
114.410 w . \\
114.375 w . \\
114.376 w .\end{array}$ & $\begin{array}{l}010 \\
010 \\
010 \\
010 \\
010\end{array}$ & $\begin{array}{l}c \\
c \\
c \\
C \\
c\end{array}$ & $\begin{array}{l}370 \\
370 \\
370 \\
370 \\
370\end{array}$ & $\begin{array}{l}\cdots \\
\cdots \\
\cdots \\
\cdots\end{array}$ & $\begin{array}{l}\cdots \\
\cdots \\
\cdots \\
\cdots\end{array}$ & $\begin{array}{l}\text { 3. } 3 \mathrm{ML} \\
\text { 3. } 3 \mathrm{ML} \\
\text { 3. } 2 \mathrm{ML} \\
\text { 3. } 9 \mathrm{ML} \\
\text { 3. } 4 \mathrm{ML}\end{array}$ & $\begin{array}{l}\text { GS } \\
\text { GS } \\
\text { GS } \\
\text { GS } \\
\text { GS }\end{array}$ & $\begin{array}{l}\cdots \\
\cdots \\
\cdots \\
\cdots\end{array}$ & $\begin{array}{l}\cdots \\
\cdots \\
\cdots \\
\cdots \\
\cdots\end{array}$ & $\begin{array}{l}\cdots \\
\cdots \\
\cdots \\
\cdots \\
\cdots\end{array}$ & $\begin{array}{l}\cdots \\
\cdots \\
\cdots \\
\cdots\end{array}$ \\
\hline $\begin{array}{l}1984 \\
1984 \\
1984 \\
1984 \\
1984\end{array}$ & $\begin{array}{l}\text { APR } \\
\text { APR } \\
\text { APR } \\
\text { APR } \\
\text { APR }\end{array}$ & $\begin{array}{l}03 \\
03 \\
10 \\
10 \\
11\end{array}$ & $\begin{array}{l}00 \\
06 \\
02 \\
14 \\
08\end{array}$ & $\begin{array}{l}07 \\
58 \\
10 \\
30 \\
23\end{array}$ & $\begin{array}{l}26.7 \\
08.1 \\
18.8 \\
54.9 \\
20.9\end{array}$ & $\begin{array}{l}44.297 \mathrm{~N} . \\
44.200 \mathrm{~N} \\
44.654 \mathrm{~N} . \\
44.270 \mathrm{~N} \\
44.297 \mathrm{~N}\end{array}$ & $\begin{array}{l}114.507 w . \\
114.161 w . \\
114.310 w . \\
114.365 w . \\
114.295 w .\end{array}$ & $\begin{array}{l}010 \\
010 \\
010 \\
010 \\
010\end{array}$ & $\begin{array}{l}\mathrm{C} \\
\mathrm{C} \\
\mathrm{C} \\
\mathrm{C} \\
\mathrm{C}\end{array}$ & $\begin{array}{l}370 \\
370 \\
370 \\
370 \\
370\end{array}$ & $\begin{array}{l}\cdots \\
\cdots \\
\cdots \\
\cdots\end{array}$ & $\begin{array}{l}\cdots \\
\cdots \\
\cdots \\
\cdots\end{array}$ & $\begin{array}{l}\text { 3. } 1 \mathrm{ML} \\
\text { 3. } \mathrm{OML} \\
\text { 3. } 1 \mathrm{ML} \\
\text { 3. } 2 \mathrm{ML} \\
\text { 3. } 4 \mathrm{ML}\end{array}$ & $\begin{array}{l}\text { MMT } \\
\text { MMT } \\
\text { GS } \\
\text { MMT } \\
\text { MMT }\end{array}$ & $\begin{array}{l}\cdots \\
\cdots \\
\cdots \\
\cdots\end{array}$ & $\begin{array}{l}\cdots \\
\cdots \\
\cdots \\
\cdots \\
\cdots\end{array}$ & $\begin{array}{l}\cdots \\
\cdots \\
F \\
F\end{array}$ & $\begin{array}{c}\ldots \\
370 \\
370 \\
\ldots\end{array}$ \\
\hline $\begin{array}{l}1984 \\
1984 \\
1984\end{array}$ & $\begin{array}{l}\text { APR } \\
\text { APR } \\
\text { APR }\end{array}$ & $\begin{array}{l}11 \\
12 \\
13\end{array}$ & $\begin{array}{l}15 \\
09 \\
01\end{array}$ & $\begin{array}{l}51 \\
08 \\
14\end{array}$ & $\begin{array}{l}55.9 \\
02.0 \\
13.5\end{array}$ & $\begin{array}{l}44.774 N \\
44.297 N \\
44.281 N\end{array}$ & $\begin{array}{l}114.469 w . \\
114.280 w . \\
114.193 w .\end{array}$ & $\begin{array}{l}010 \\
010 \\
010\end{array}$ & $\begin{array}{l}B \\
C \\
C\end{array}$ & $\begin{array}{l}370 \\
370 \\
370\end{array}$ & $\begin{array}{c}3.7 \\
\ldots \\
\ldots\end{array}$ & $\begin{array}{l}\cdots \\
\cdots \\
\cdots\end{array}$ & $\begin{array}{l}\text { 4. } 1 \mathrm{ML} \\
\text { 3. } \mathrm{OML} \\
\text { 3. } 4 \mathrm{ML}\end{array}$ & $\begin{array}{l}\text { GS } \\
\text { MMT } \\
\text { MMT }\end{array}$ & $\begin{array}{l}\cdots \\
\cdots \\
\cdots\end{array}$ & $\begin{array}{l}\cdots \\
\cdots \\
\cdots\end{array}$ & $\begin{array}{r}\text { IV } \\
F \\
\ldots\end{array}$ & $\begin{array}{r}370 \\
370 \\
\ldots\end{array}$ \\
\hline
\end{tabular}




\begin{tabular}{|c|c|c|c|c|c|c|c|c|c|c|c|c|c|c|c|c|c|c|}
\hline $\begin{array}{l}1984 \\
1984\end{array}$ & $\begin{array}{l}\text { APR } \\
\text { APR }\end{array}$ & $\begin{array}{l}13 \\
13\end{array}$ & $\begin{array}{l}07 \\
12\end{array}$ & $\begin{array}{l}16 \\
16\end{array}$ & $\begin{array}{l}39.5 \\
05.4\end{array}$ & $\begin{array}{l}44.727 N \\
44.742 N\end{array}$ & $\begin{array}{l}114.433 W . \\
114.424 W .\end{array}$ & $\begin{array}{l}010 \\
010\end{array}$ & $\begin{array}{l}\mathrm{C} \\
\mathrm{C}\end{array}$ & $\begin{array}{l}370 \\
370\end{array}$ & $\begin{array}{l}\cdots \\
\cdots\end{array}$ & $\begin{array}{l}\cdots \\
\cdots\end{array}$ & $\begin{array}{l}\text { 3. OML } \\
\text { 3. 1ML }\end{array}$ & $\begin{array}{l}\text { GS } \\
\text { GS }\end{array}$ & $\begin{array}{l}\cdots \\
\cdots\end{array}$ & $\cdots$ & $\begin{array}{l}\cdots \\
\cdots\end{array}$ & $\begin{array}{l}\cdots \\
\cdots\end{array}$ \\
\hline $\begin{array}{l}1984 \\
1984 \\
1984 \\
1984 \\
1984\end{array}$ & $\begin{array}{l}\text { APR } \\
\text { APR } \\
\text { APR } \\
\text { APR } \\
\text { APR }\end{array}$ & $\begin{array}{l}18 \\
19 \\
20 \\
23 \\
29\end{array}$ & $\begin{array}{l}01 \\
01 \\
17 \\
05 \\
04\end{array}$ & $\begin{array}{l}15 \\
58 \\
25 \\
54 \\
42\end{array}$ & $\begin{array}{l}09.4 \\
49.5 \\
31.2 \\
14.6 \\
55.8\end{array}$ & $\begin{array}{l}44.342 N \\
44.363 N \\
44.608 N \\
44.133 N \\
44.244 N\end{array}$ & $\begin{array}{l}114.099 w . \\
114.137 w . \\
114.408 w . \\
113.867 w . \\
114.135 w .\end{array}$ & $\begin{array}{l}010 \\
010 \\
010 \\
010 \\
010\end{array}$ & $\begin{array}{l}B \\
C \\
C \\
D \\
C\end{array}$ & $\begin{array}{l}370 \\
370 \\
370 \\
370 \\
370\end{array}$ & $\begin{array}{l}\cdots \\
\cdots \\
\cdots \\
\cdots\end{array}$ & $\begin{array}{l}\cdots \\
\cdots \\
\cdots \\
\cdots\end{array}$ & $\begin{array}{l}\text { 4. } 0 \mathrm{ML} \\
3.3 \mathrm{ML} \\
3.0 \mathrm{ML} \\
3.3 \mathrm{ML} \\
3.4 \mathrm{ML}\end{array}$ & $\begin{array}{l}\text { GS } \\
\text { GS } \\
\text { MMT } \\
\text { MMT } \\
\text { GS }\end{array}$ & $\begin{array}{l}\cdots \\
\cdots \\
\cdots \\
\cdots\end{array}$ & $\begin{array}{l}\cdots \\
\cdots \\
\cdots \\
\cdots\end{array}$ & $\begin{array}{l}\cdots \\
\cdots \\
\cdots \\
\cdots \\
\cdots\end{array}$ & $\begin{array}{l}\cdots \\
\cdots \\
\cdots \\
\cdots\end{array}$ \\
\hline $\begin{array}{l}1984 \\
1984 \\
1984 \\
1984 \\
1984\end{array}$ & $\begin{array}{l}\text { MAY } \\
\text { MAY } \\
\text { JUN } \\
\text { JUN } \\
\text { JUN }\end{array}$ & $\begin{array}{l}16 \\
30 \\
15 \\
26 \\
30\end{array}$ & $\begin{array}{l}18 \\
04 \\
23 \\
19 \\
23\end{array}$ & $\begin{array}{l}02 \\
11 \\
04 \\
15 \\
39\end{array}$ & $\begin{array}{l}20.0 \\
01.7 \\
32.7 \\
35.9 \\
07.5\end{array}$ & $\begin{array}{l}44.302 \mathrm{~N} \\
44.264 \mathrm{~N} \\
46.641 \mathrm{~N} \\
44.221 \mathrm{~N} \\
44.651 \mathrm{~N}\end{array}$ & $\begin{array}{l}114.126 w . \\
114.136 w . \\
116.245 W . \\
114.046 W . \\
194.396 w .\end{array}$ & $\begin{array}{l}010 \\
010 \\
001 \\
010 \\
010\end{array}$ & $\begin{array}{l}c \\
C \\
c \\
C \\
C\end{array}$ & $\begin{array}{l}370 \\
370 \\
370 \\
370 \\
370\end{array}$ & $\begin{array}{l}\cdots \\
\cdots \\
\cdots \\
\cdots\end{array}$ & $\begin{array}{l}\cdots \\
\cdots \\
\cdots \\
\cdots\end{array}$ & $\begin{array}{l}3.2 \mathrm{ML} \\
3.5 \mathrm{ML} \\
2.5 \mathrm{ML} \\
3.5 \mathrm{ML} \\
3.8 \mathrm{ML}\end{array}$ & $\begin{array}{l}\text { GS } \\
\text { GS } \\
\text { MMT } \\
\text { GS } \\
\text { GS }\end{array}$ & $\begin{array}{l}\cdots \\
\cdots \\
\cdots \\
\cdots\end{array}$ & $\begin{array}{l}\cdots \\
\cdots \\
\cdots \\
\cdots \\
\cdots\end{array}$ & $\begin{array}{l}\cdots \\
\cdots \\
\cdots \\
\cdots \\
\cdots\end{array}$ & $\begin{array}{l}\cdots \\
\cdots \\
\cdots \\
\cdots\end{array}$ \\
\hline $\begin{array}{l}1984 \\
1984 \\
1984 \\
1984 \\
1984\end{array}$ & $\begin{array}{l}\text { JUL } \\
\text { JUL } \\
\text { JUL } \\
\text { JUL } \\
\text { JUL }\end{array}$ & $\begin{array}{l}95 \\
14 \\
16 \\
29 \\
30\end{array}$ & $\begin{array}{l}19 \\
17 \\
01 \\
11 \\
23\end{array}$ & $\begin{array}{l}32 \\
46 \\
34 \\
54 \\
06\end{array}$ & $\begin{array}{l}59.0 \\
02.1 \\
52.8 \\
14.3 \\
48.2\end{array}$ & $\begin{array}{l}44.574 \mathrm{~N} \\
44.628 \mathrm{~N} . \\
44.302 \mathrm{~N} \\
44.620 \mathrm{~N} \\
48.872 \mathrm{~N}\end{array}$ & $\begin{array}{l}114.286 W . \\
114.293 W . \\
114.333 W . \\
114.490 W . \\
116.204 W .\end{array}$ & $\begin{array}{l}010 \\
010 \\
010 \\
010 \\
011\end{array}$ & $\begin{array}{l}C \\
C \\
C \\
C \\
B\end{array}$ & $\begin{array}{l}370 \\
370 \\
370 \\
370 \\
370\end{array}$ & $\begin{array}{l}\cdots \\
\cdots \\
\cdots \\
\cdots\end{array}$ & $\begin{array}{l}\cdots \\
\cdots \\
\cdots \\
\cdots\end{array}$ & $\begin{array}{l}\text { 3. } 4 \mathrm{ML} \\
3.6 \mathrm{ML} \\
3.3 \mathrm{ML} \\
3.4 \mathrm{ML} \\
3.4 \mathrm{ML}\end{array}$ & $\begin{array}{l}\text { GS } \\
\text { GS } \\
\text { GS } \\
\text { GS } \\
\text { MMT }\end{array}$ & $\begin{array}{l}\cdots \\
\cdots \\
\cdots \\
\cdots\end{array}$ & $\begin{array}{l}\cdots \\
\cdots \\
\cdots \\
\cdots \\
\cdots\end{array}$ & $\begin{array}{l}\cdots \\
\cdots \\
\cdots \\
\cdots \\
\cdots\end{array}$ & $\begin{array}{l}\cdots \\
\cdots \\
\cdots \\
\cdots\end{array}$ \\
\hline $\begin{array}{l}1984 \\
1984 \\
1984 \\
1984 \\
1984\end{array}$ & $\begin{array}{l}\text { AUG } \\
\text { AUG } \\
\text { AUG } \\
\text { AUG } \\
\text { AUG }\end{array}$ & $\begin{array}{l}15 \\
21 \\
22 \\
22 \\
22\end{array}$ & $\begin{array}{l}22 \\
23 \\
09 \\
10 \\
10\end{array}$ & $\begin{array}{l}07 \\
42 \\
46 \\
10 \\
48\end{array}$ & $\begin{array}{l}52.4 \\
13.7 \\
30.2 \\
05.7 \\
24.2\end{array}$ & $\begin{array}{l}44.126 \mathrm{~N} \\
44.087 \mathrm{~N} . \\
44.467 \mathrm{~N} \\
44.082 \mathrm{~N} \\
44.454 \mathrm{~N}\end{array}$ & $\begin{array}{l}114.717 w . \\
114.759 w . \\
114.008 w . \\
114.687 w . \\
114.153 W .\end{array}$ & $\begin{array}{l}010 \\
010 \\
010 \\
010 \\
010\end{array}$ & $\begin{array}{l}C \\
C \\
B \\
C \\
C\end{array}$ & $\begin{array}{l}370 \\
370 \\
370 \\
370 \\
370\end{array}$ & 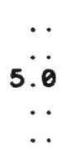 & $\begin{array}{c}\ldots \\
5.1 \\
\ldots \\
\ldots\end{array}$ & $\begin{array}{l}3.4 \mathrm{ML} \\
3.2 \mathrm{ML} \\
5.8 \mathrm{ML} \\
3.0 \mathrm{ML} \\
3.3 \mathrm{ML}\end{array}$ & $\begin{array}{l}\text { GS } \\
\text { GS } \\
\text { UU } \\
\text { GS } \\
\text { GS }\end{array}$ & $\begin{array}{l}\cdots \\
\cdots \\
\cdots \\
\cdots\end{array}$ & $\begin{array}{l}\cdots \\
\cdots \\
\cdots \\
\cdots \\
\cdots\end{array}$ & $\begin{array}{l}\cdots \\
\cdots \\
\cdots \\
\cdots\end{array}$ & $\begin{array}{c}\ldots \\
370 \\
\ldots \\
\cdots\end{array}$ \\
\hline $\begin{array}{l}1984 \\
1984 \\
1984 \\
1984 \\
1984\end{array}$ & $\begin{array}{l}\text { AUG } \\
\text { AUG } \\
\text { AUG } \\
\text { AUG } \\
\text { AUG }\end{array}$ & $\begin{array}{l}22 \\
22 \\
22 \\
22 \\
22\end{array}$ & $\begin{array}{l}10 \\
10 \\
11 \\
11 \\
11\end{array}$ & $\begin{array}{l}50 \\
52 \\
09 \\
44 \\
52\end{array}$ & $\begin{array}{l}25.0 \\
01.1 \\
19.7 \\
24.9 \\
30.8\end{array}$ & $\begin{array}{l}44.452 \mathrm{~N} \\
44.481 \mathrm{~N} . \\
44.468 \mathrm{~N} \\
44.419 \mathrm{~N} . \\
44.186 \mathrm{~N} .\end{array}$ & $\begin{array}{l}114.113 w . \\
114.204 W . \\
114.084 w . \\
114.118 w . \\
114.658 w .\end{array}$ & $\begin{array}{l}010 \\
010 \\
010 \\
010 \\
010\end{array}$ & $\begin{array}{l}c \\
c \\
c \\
C \\
c\end{array}$ & $\begin{array}{l}370 \\
370 \\
370 \\
370 \\
370\end{array}$ & $\begin{array}{l}\cdots \\
\cdots \\
\cdots \\
\cdots\end{array}$ & $\begin{array}{l}\cdots \\
\cdots \\
\cdots \\
\cdots\end{array}$ & $\begin{array}{l}\text { 3. } 3 \mathrm{ML} \\
\text { 4. } \mathrm{OML} \\
\text { 3. } 3 \mathrm{ML} \\
\text { 3. } 2 \mathrm{ML} \\
\text { 3. } 2 \mathrm{ML}\end{array}$ & $\begin{array}{l}\text { GS } \\
\text { GS } \\
\text { GS } \\
\text { GS } \\
\text { GS }\end{array}$ & $\begin{array}{l}\cdots \\
\cdots \\
\cdots \\
\cdots\end{array}$ & $\begin{array}{l}\cdots \\
\cdots \\
\cdots \\
\cdots \\
\cdots\end{array}$ & $\begin{array}{l}\cdots \\
\cdots \\
\cdots \\
\cdots \\
\cdots\end{array}$ & $\begin{array}{l}\cdots \\
\cdots \\
\cdots \\
\cdots\end{array}$ \\
\hline $\begin{array}{l}1984 \\
1984 \\
1984 \\
1984 \\
1984\end{array}$ & $\begin{array}{l}\text { AUG } \\
\text { AUG } \\
\text { AUG } \\
\text { AUG } \\
\text { AUG }\end{array}$ & $\begin{array}{l}22 \\
22 \\
22 \\
23 \\
23\end{array}$ & $\begin{array}{l}12 \\
13 \\
15 \\
00 \\
06\end{array}$ & $\begin{array}{l}06 \\
34 \\
35 \\
01 \\
13\end{array}$ & $\begin{array}{l}31.6 \\
21.6 \\
41.5 \\
58.1 \\
33.2\end{array}$ & $\begin{array}{l}44.377 \mathrm{~N} . \\
44.471 \mathrm{~N} \\
44.419 \mathrm{~N} \\
44.369 \mathrm{~N} \\
44.445 \mathrm{~N}\end{array}$ & $\begin{array}{l}114.181 w . \\
114.152 W . \\
114.094 W . \\
114.203 W . \\
114.089 W .\end{array}$ & $\begin{array}{l}010 \\
010 \\
010 \\
010 \\
010\end{array}$ & $\begin{array}{l}\text { C } \\
C \\
D \\
C \\
C\end{array}$ & $\begin{array}{l}370 \\
370 \\
370 \\
370 \\
370\end{array}$ & $\begin{array}{l}\cdots \\
\cdots \\
\cdots \\
\cdots\end{array}$ & $\begin{array}{l}\cdots \\
\cdots \\
\cdots \\
\cdots\end{array}$ & $\begin{array}{l}\text { 3. } 1 \mathrm{ML} \\
\text { 4. } 1 \mathrm{ML} \\
\text { 3. } 2 \mathrm{ML} \\
\text { 3. } 2 \mathrm{ML} \\
\text { 3. } 4 \mathrm{ML}\end{array}$ & $\begin{array}{l}\text { GS } \\
\text { GS } \\
\text { GS } \\
\text { GS } \\
\text { GS }\end{array}$ & $\begin{array}{l}\cdots \\
\cdots \\
\cdots \\
\cdots\end{array}$ & $\begin{array}{l}\cdots \\
\cdots \\
\cdots \\
\cdots\end{array}$ & $\begin{array}{l}\cdots \\
\cdots \\
\cdots \\
\cdots \\
\cdots\end{array}$ & $\begin{array}{l}\cdots \\
\cdots \\
\cdots \\
\cdots\end{array}$ \\
\hline $\begin{array}{l}1984 \\
1984 \\
1984 \\
1984 \\
1984\end{array}$ & $\begin{array}{l}\text { AUG } \\
\text { AUG } \\
\text { AUG } \\
\text { AUG } \\
\text { AUG }\end{array}$ & $\begin{array}{l}23 \\
24 \\
25 \\
25 \\
27\end{array}$ & $\begin{array}{l}13 \\
13 \\
07 \\
17 \\
02\end{array}$ & $\begin{array}{l}21 \\
53 \\
43 \\
43 \\
12\end{array}$ & $\begin{array}{l}53.0 \\
48.2 \\
34.0 \\
53.0 \\
08.8\end{array}$ & $\begin{array}{l}44.462 \mathrm{~N} \\
44.437 \mathrm{~N} \\
44.459 \mathrm{~N} . \\
44.416 \mathrm{~N} \\
44.420 \mathrm{~N}\end{array}$ & $\begin{array}{l}114.137 W . \\
114.063 W . \\
114.141 W . \\
114.100 W . \\
114.174 W .\end{array}$ & $\begin{array}{l}010 \\
010 \\
010 \\
010 \\
010\end{array}$ & $\begin{array}{l}c \\
c \\
c \\
c \\
c\end{array}$ & $\begin{array}{l}370 \\
370 \\
370 \\
370 \\
370\end{array}$ & $\begin{array}{l}\cdots \\
\cdots \\
\cdots \\
\cdots\end{array}$ & $\begin{array}{l}\cdots \\
\cdots \\
\cdots \\
\cdots\end{array}$ & $\begin{array}{l}\text { 3. } 8 \mathrm{ML} \\
\text { 3. } 4 \mathrm{ML} \\
\text { 3. } 1 \mathrm{ML} \\
\text { 3. } 9 \mathrm{ML} \\
\text { 3. } 1 \mathrm{ML}\end{array}$ & $\begin{array}{l}\text { GS } \\
\text { GS } \\
\text { GS } \\
\text { GS } \\
\text { GS }\end{array}$ & $\begin{array}{l}\cdots \\
\cdots \\
\cdots \\
\cdots\end{array}$ & $\begin{array}{l}\ldots \\
\cdots \\
\cdots \\
\cdots\end{array}$ & $\begin{array}{l}\cdots \\
\cdots \\
\cdots \\
\cdots \\
\cdots\end{array}$ & $\begin{array}{l}\cdots \\
\cdots \\
\cdots \\
\cdots\end{array}$ \\
\hline $\begin{array}{l}1984 \\
1984 \\
1984 \\
1984 \\
1984\end{array}$ & $\begin{array}{l}\text { AUG } \\
\text { AUG } \\
\text { AUG } \\
\text { AUG } \\
\text { AUG }\end{array}$ & $\begin{array}{l}28 \\
28 \\
29 \\
30 \\
30\end{array}$ & $\begin{array}{l}11 \\
20 \\
09 \\
18 \\
21\end{array}$ & $\begin{array}{l}19 \\
40 \\
47 \\
42 \\
06\end{array}$ & $\begin{array}{l}44.2 \\
30.0 \\
51.5 \\
27.6 \\
20.7\end{array}$ & $\begin{array}{l}44.456 \mathrm{~N} \\
44.444 \mathrm{~N} \\
44.386 \mathrm{~N} \\
44.452 \mathrm{~N} \\
44.455 \mathrm{~N}\end{array}$ & $\begin{array}{l}114.138 W . \\
114.172 W . \\
114.060 W . \\
114.126 W . \\
114.154 W .\end{array}$ & $\begin{array}{l}010 \\
010 \\
010 \\
010 \\
010\end{array}$ & $\begin{array}{l}c \\
c \\
c \\
c \\
c\end{array}$ & $\begin{array}{l}370 \\
370 \\
370 \\
370 \\
370\end{array}$ & $\begin{array}{l}\cdots \\
\cdots \\
\cdots \\
\cdots\end{array}$ & $\begin{array}{l}\cdots \\
\cdots \\
\cdots \\
\cdots\end{array}$ & $\begin{array}{l}3.1 \mathrm{ML} \\
3.4 \mathrm{ML} \\
3.3 \mathrm{ML} \\
3.5 \mathrm{ML} \\
3.9 \mathrm{ML}\end{array}$ & $\begin{array}{l}\text { GS } \\
\text { GS } \\
\text { GS } \\
\text { GS } \\
\text { GS }\end{array}$ & $\begin{array}{l}\cdots \\
\cdots \\
\cdots \\
\cdots\end{array}$ & $\begin{array}{l}\ldots \\
\cdots \\
\cdots \\
\cdots \\
\cdots\end{array}$ & $\begin{array}{l}\cdots \\
\cdots \\
\cdots \\
F \\
F\end{array}$ & $\begin{array}{c}\cdots \\
\ldots \\
370 \\
370\end{array}$ \\
\hline $\begin{array}{l}1984 \\
1984 \\
1984 \\
1984 \\
1984\end{array}$ & $\begin{array}{l}\text { SEP } \\
\text { SEP } \\
\text { SEP } \\
\text { SEP } \\
\text { SEP }\end{array}$ & $\begin{array}{l}01 \\
03 \\
04 \\
04 \\
04\end{array}$ & $\begin{array}{l}14 \\
20 \\
05 \\
05 \\
14\end{array}$ & $\begin{array}{l}11 \\
28 \\
50 \\
57 \\
10\end{array}$ & $\begin{array}{l}18.3 \\
00.1 \\
41.3 \\
21.8 \\
03.6\end{array}$ & $\begin{array}{l}44.439 \mathrm{~N} \\
44.359 \mathrm{~N} \\
44.449 \mathrm{~N} \\
44.466 \mathrm{~N} \\
44.491 \mathrm{~N}\end{array}$ & $\begin{array}{l}114.117 w . \\
114.126 W . \\
114.107 W . \\
114.115 W . \\
114.127 W .\end{array}$ & $\begin{array}{l}010 \\
010 \\
010 \\
010 \\
010\end{array}$ & $\begin{array}{l}C \\
C \\
B \\
C \\
C\end{array}$ & $\begin{array}{l}370 \\
370 \\
370 \\
370 \\
370\end{array}$ & $\begin{array}{l}\cdots \\
\cdots \\
\cdots \\
\cdots\end{array}$ & $\begin{array}{l}\cdots \\
\cdots \\
\cdots \\
\cdots \\
\cdots\end{array}$ & $\begin{array}{l}\text { 3. } 1 \mathrm{ML} \\
\text { 3. } 2 \mathrm{ML} \\
\text { 3. } 7 \mathrm{ML} \\
\text { 3. } 1 \mathrm{ML} \\
2.9 \mathrm{ML}\end{array}$ & $\begin{array}{l}\text { GS } \\
\text { GS } \\
\text { GS } \\
\text { GS } \\
\text { GS }\end{array}$ & $\begin{array}{l}\cdots \\
\cdots \\
\cdots \\
\cdots\end{array}$ & $\begin{array}{l}\cdots \\
\cdots \\
\cdots \\
\cdots \\
\cdots\end{array}$ & $\begin{array}{c}\cdots \\
\text { IV } \\
\cdots \\
\cdots\end{array}$ & $\begin{array}{c}\ldots \\
370 \\
\ldots\end{array}$ \\
\hline $\begin{array}{l}1984 \\
1984 \\
1984 \\
1984 \\
1984\end{array}$ & $\begin{array}{l}\text { SEP } \\
\text { SEP } \\
\text { SEP } \\
\text { SEP } \\
\text { SEP }\end{array}$ & $\begin{array}{l}05 \\
05 \\
05 \\
06 \\
08\end{array}$ & $\begin{array}{l}09 \\
17 \\
20 \\
19 \\
06\end{array}$ & $\begin{array}{l}39 \\
38 \\
28 \\
50 \\
16\end{array}$ & $\begin{array}{l}54.8 \\
58.4 \\
01.2 \\
22.3 \\
40.1\end{array}$ & $\begin{array}{l}44.432 \mathrm{~N} \\
44.421 \mathrm{~N} \\
44.218 \mathrm{~N} \\
44.450 \mathrm{~N} \\
44.439 \mathrm{~N}\end{array}$ & $\begin{array}{l}114.154 W . \\
114.183 W . \\
114.134 W . \\
114.077 W . \\
114.154 W .\end{array}$ & $\begin{array}{l}010 \\
010 \\
010 \\
010 \\
010\end{array}$ & $\begin{array}{l}C \\
B \\
D \\
C \\
B\end{array}$ & $\begin{array}{l}370 \\
370 \\
370 \\
370 \\
370\end{array}$ & $\begin{array}{l}\cdots \\
\cdots \\
\cdots \\
5.0\end{array}$ & $\begin{array}{c}\cdots \\
\ldots \\
\cdots \\
4 .\end{array}$ & $\begin{array}{l}\text { 3. } 4 M L \\
\text { 3. } 7 \mathrm{ML} \\
\text { 3. } 1 \mathrm{ML} \\
\text { 3. } 4 \mathrm{ML} \\
\text { 4. } 3 \mathrm{MD}\end{array}$ & $\begin{array}{l}\text { GS } \\
\text { GS } \\
\text { GS } \\
\text { MMT } \\
\text { UU }\end{array}$ & $\begin{array}{l}\cdots \\
\cdots \\
\cdots \\
\cdots\end{array}$ & $\begin{array}{l}\cdots \\
\cdots \\
\cdots \\
\cdots\end{array}$ & $\begin{array}{c}\cdots \dot{F} \\
\cdots \\
\dot{I V}\end{array}$ & $\begin{array}{c}370 \\
\ldots \\
370\end{array}$ \\
\hline $\begin{array}{l}1984 \\
1984 \\
1984 \\
1984 \\
1984\end{array}$ & $\begin{array}{l}\text { SEP } \\
\text { SEP } \\
\text { SEP } \\
\text { SEP } \\
\text { SEP }\end{array}$ & $\begin{array}{l}08 \\
08 \\
08 \\
08 \\
08\end{array}$ & $\begin{array}{l}06 \\
08 \\
08 \\
08 \\
08\end{array}$ & $\begin{array}{l}26 \\
01 \\
03 \\
08 \\
16\end{array}$ & $\begin{array}{l}46.0 \\
02.3 \\
16.8 \\
32.9 \\
28.1\end{array}$ & $\begin{array}{l}44.418 \mathrm{~N} \\
44.284 \mathrm{~N} \\
44.415 \mathrm{~N} \\
44.409 \mathrm{~N} \\
44.441 \mathrm{~N}\end{array}$ & $\begin{array}{l}114.136 W . \\
114.256 W . \\
114.062 W . \\
114.149 W . \\
114.081 W .\end{array}$ & $\begin{array}{l}010 \\
010 \\
010 \\
010 \\
010\end{array}$ & $\begin{array}{l}C \\
C \\
C \\
D \\
C\end{array}$ & $\begin{array}{l}370 \\
370 \\
370 \\
370 \\
370\end{array}$ & $\begin{array}{l}\cdots \\
\cdots \\
\cdots \\
\cdots\end{array}$ & $\begin{array}{l}\cdots \\
\cdots \\
\cdots \\
\cdots\end{array}$ & $\begin{array}{l}\text { 3. } 5 \mathrm{ML} \\
\text { 3. } \mathrm{OML} \\
3.5 \mathrm{ML} \\
\text { 3. } 3 \mathrm{ML} \\
\text { 3. } 3 \mathrm{ML}\end{array}$ & $\begin{array}{l}\text { GS } \\
\text { GS } \\
\text { GS } \\
\text { GS } \\
\text { GS }\end{array}$ & $\begin{array}{l}\cdots \\
\cdots \\
\cdots \\
\cdots\end{array}$ & $\begin{array}{l}\cdots \\
\cdots \\
\cdots \\
\cdots\end{array}$ & $\begin{array}{l}\cdots \\
\cdots \\
\cdots \\
\cdots \\
\cdots\end{array}$ & $\begin{array}{l}\cdots \\
\cdots \\
\cdots \\
\cdots\end{array}$ \\
\hline 1984 & SEP & 08 & 08 & 35 & 02.9 & $44.441 \mathrm{~N}$ & $114.141 W$ & 010 & C & 370 & $\ldots$ & $\cdots$ & 3. $1 \mathrm{ML}$ & GS & .. & $\ldots$ & $\ldots$ & $\cdots$ \\
\hline
\end{tabular}




\begin{tabular}{|c|c|c|c|c|c|c|c|c|c|c|c|c|c|c|c|c|c|c|}
\hline $\begin{array}{l}1984 \\
1984 \\
1984 \\
1984\end{array}$ & $\begin{array}{l}\text { SEP } \\
\text { SEP } \\
\text { SEP } \\
\text { SEP }\end{array}$ & $\begin{array}{l}08 \\
08 \\
08 \\
08\end{array}$ & $\begin{array}{l}08 \\
09 \\
09 \\
11\end{array}$ & $\begin{array}{l}55 \\
22 \\
27 \\
36\end{array}$ & $\begin{array}{l}50.0 \\
26.4 \\
28.3 \\
13.7\end{array}$ & $\begin{array}{l}44.444 \mathrm{~N} \\
44.441 \mathrm{~N} \\
44.438 \mathrm{~N} \\
44.420 \mathrm{~N}\end{array}$ & $\begin{array}{l}114.152 W . \\
114.115 W . \\
114.091 W . \\
114.150 w .\end{array}$ & $\begin{array}{l}010 \\
010 \\
010 \\
010\end{array}$ & $\begin{array}{l}B \\
C \\
C \\
B\end{array}$ & $\begin{array}{l}370 \\
370 \\
370 \\
370\end{array}$ & $\begin{array}{l}\cdots \\
\cdots \\
\cdots\end{array}$ & $\begin{array}{l}\cdots \\
\cdots \\
\cdots\end{array}$ & $\begin{array}{l}\text { 3.9ML } \\
\text { 3. } \mathrm{OML} \\
\text { 3. } \mathrm{OML} \\
\text { 4. } \mathrm{OML}\end{array}$ & $\begin{array}{l}\text { GS } \\
\text { GS } \\
\text { GS } \\
\text { GS }\end{array}$ & $\begin{array}{l}\cdots \\
\cdots \\
\cdots\end{array}$ & $\begin{array}{l}\cdots \\
\cdots \\
\cdots \\
\cdots\end{array}$ & $\begin{array}{c}\text { III } \\
\cdots \\
\cdots \\
\ddot{F}\end{array}$ & $\begin{array}{r}370 \\
\ldots \\
370\end{array}$ \\
\hline $\begin{array}{l}1984 \\
1984 \\
1984 \\
1984 \\
1984\end{array}$ & $\begin{array}{l}\text { SEP } \\
\text { SEP } \\
\text { SEP } \\
\text { SEP } \\
\text { SEP }\end{array}$ & $\begin{array}{l}08 \\
13 \\
14 \\
15 \\
18\end{array}$ & $\begin{array}{l}13 \\
11 \\
10 \\
02 \\
15\end{array}$ & $\begin{array}{l}56 \\
12 \\
55 \\
55 \\
09\end{array}$ & $\begin{array}{l}37.7 \\
48.9 \\
02.4 \\
02.6 \\
58.0\end{array}$ & $\begin{array}{l}44.416 \mathrm{~N} \\
44.434 \mathrm{~N} \\
45.250 \mathrm{~N} \\
47.460 \mathrm{~N} \\
44.408 \mathrm{~N}\end{array}$ & $\begin{array}{l}114.147 W . \\
114.150 W . \\
114.621 W . \\
115.987 W . \\
114.124 W .\end{array}$ & $\begin{array}{l}010 \\
010 \\
010 \\
001 \\
010\end{array}$ & $\begin{array}{l}B \\
B \\
B \\
C \\
B\end{array}$ & $\begin{array}{l}370 \\
370 \\
370 \\
370 \\
370\end{array}$ & $\begin{array}{c}4.4 \\
\ldots \\
\ldots \\
\ldots \\
\ldots\end{array}$ & $\begin{array}{l}\cdots \\
\cdots \\
\cdots \\
\cdots\end{array}$ & $\begin{array}{l}\text { 4. } 6 \mathrm{MD} \\
\text { 3.9ML } \\
\text { 3. } 6 \mathrm{ML} \\
\text { 2.8ML } \\
\text { 4. } 9 \mathrm{ML}\end{array}$ & $\begin{array}{l}\text { MMT } \\
\text { GS } \\
\text { GS } \\
\text { GS } \\
\text { GS }\end{array}$ & $\begin{array}{l}\cdots \\
\cdots \\
\cdots \\
\cdots\end{array}$ & $\begin{array}{l}\ldots \\
\ldots \\
\ldots \\
\ldots\end{array}$ & $\begin{array}{r}\text { I I I } \\
\text { IV } \\
\ldots \\
\text { F } \\
\text { F }\end{array}$ & $\begin{array}{r}370 \\
370 \\
370 \\
370\end{array}$ \\
\hline $\begin{array}{l}1984 \\
1984 \\
1984 \\
1984 \\
1984\end{array}$ & $\begin{array}{l}\text { SEP } \\
\text { SEP } \\
\text { SEP } \\
\text { SEP } \\
\text { SEP }\end{array}$ & $\begin{array}{l}18 \\
19 \\
19 \\
21 \\
21\end{array}$ & $\begin{array}{l}16 \\
01 \\
15 \\
08 \\
18\end{array}$ & $\begin{array}{l}27 \\
32 \\
33 \\
40 \\
21\end{array}$ & $\begin{array}{l}30.6 \\
06.9 \\
22.6 \\
40.2 \\
55.5\end{array}$ & $\begin{array}{l}44.420 \mathrm{~N} \\
45.079 \mathrm{~N} \\
44.334 \mathrm{~N} \\
44.817 \mathrm{~N} \\
44.428 \mathrm{~N}\end{array}$ & $\begin{array}{l}114.107 W . \\
116.766 W . \\
114.205 W . \\
112.899 W . \\
114.242 W .\end{array}$ & $\begin{array}{l}010 \\
005 \\
010 \\
010 \\
010\end{array}$ & $\begin{array}{l}C \\
C \\
B \\
C \\
C\end{array}$ & $\begin{array}{l}370 \\
370 \\
370 \\
370 \\
370\end{array}$ & $\begin{array}{l}\cdots \\
\cdots \\
\cdots \\
\cdots\end{array}$ & $\begin{array}{l}\cdots \\
\cdots \\
\cdots \\
\cdots \\
\cdots\end{array}$ & $\begin{array}{l}3.2 \mathrm{ML} \\
3.4 \mathrm{ML} \\
3.5 \mathrm{ML} \\
3.6 \mathrm{ML} \\
3.4 \mathrm{ML}\end{array}$ & $\begin{array}{l}\text { GS } \\
\text { GS } \\
\text { GS } \\
\text { GS } \\
\text { GS }\end{array}$ & $\begin{array}{l}\cdots \\
\cdots \\
\cdots \\
\cdots\end{array}$ & $\begin{array}{l}\ldots \\
\ldots \\
\ldots \\
\cdots \\
\cdots\end{array}$ & $\begin{array}{l}\ldots \\
\text { II I } \\
\ldots \\
\ldots \\
\ldots\end{array}$ & $\begin{array}{r}370 \\
\ldots \\
\ldots\end{array}$ \\
\hline $\begin{array}{l}1984 \\
1984 \\
1984 \\
1984 \\
1984\end{array}$ & $\begin{array}{l}\text { SEP } \\
\text { SEP } \\
\text { SEP } \\
\text { OCT } \\
\text { OCT }\end{array}$ & $\begin{array}{l}23 \\
26 \\
29 \\
02 \\
03\end{array}$ & $\begin{array}{l}08 \\
10 \\
14 \\
09 \\
05\end{array}$ & $\begin{array}{l}19 \\
19 \\
34 \\
51 \\
32\end{array}$ & $\begin{array}{l}44.1 \\
20.6 \\
40.8 \\
31.8 \\
01.2\end{array}$ & $\begin{array}{l}44.420 \mathrm{~N} \\
44.514 \mathrm{~N} \\
44.614 \mathrm{~N} \\
44.392 \mathrm{~N} \\
44.333 \mathrm{~N}\end{array}$ & $\begin{array}{l}114.150 W . \\
114.228 W . \\
115.057 W . \\
114.184 W . \\
114.033 W .\end{array}$ & $\begin{array}{l}010 \\
010 \\
010 \\
010 \\
010\end{array}$ & $\begin{array}{l}C \\
C \\
C \\
B \\
C\end{array}$ & $\begin{array}{l}370 \\
370 \\
370 \\
370 \\
370\end{array}$ & $\begin{array}{l}\cdots \\
\cdots \\
\cdots \\
\cdots\end{array}$ & $\begin{array}{l}\cdots \\
\cdots \\
\cdots \\
\cdots\end{array}$ & $\begin{array}{l}3.4 \mathrm{ML} \\
3.0 \mathrm{ML} \\
3.3 \mathrm{ML} \\
3.6 \mathrm{ML} \\
3.2 \mathrm{ML}\end{array}$ & $\begin{array}{l}\text { GS } \\
\text { GS } \\
\text { GS } \\
\text { GS } \\
\text { GS }\end{array}$ & $\begin{array}{l}\cdots \\
\cdots \\
\cdots \\
\cdots\end{array}$ & $\begin{array}{l}\cdots \\
\cdots \\
\cdots \\
\cdots\end{array}$ & $\begin{array}{l}\ldots \\
\ldots \\
\ldots \\
\ldots \\
\cdots\end{array}$ & . \\
\hline $\begin{array}{l}1984 \\
1984 \\
1984 \\
1984 \\
1984\end{array}$ & $\begin{array}{l}\text { OCT } \\
\text { OCT } \\
\text { OCT } \\
\text { OCT } \\
\text { OCT }\end{array}$ & $\begin{array}{l}04 \\
12 \\
19 \\
25 \\
30\end{array}$ & $\begin{array}{l}17 \\
04 \\
07 \\
12 \\
23\end{array}$ & $\begin{array}{l}02 \\
49 \\
35 \\
10 \\
05\end{array}$ & $\begin{array}{l}45.2 \\
24.3 \\
09.1 \\
36.5 \\
30.6\end{array}$ & $\begin{array}{l}44.282 \mathrm{~N} \\
44.315 \mathrm{~N} \\
44.424 \mathrm{~N} \\
44.340 \mathrm{~N} \\
44.430 \mathrm{~N}\end{array}$ & $\begin{array}{l}114.090 W . \\
114.083 w . \\
114.021 W . \\
114.157 w . \\
114.112 W .\end{array}$ & $\begin{array}{l}010 \\
010 \\
010 \\
010 \\
010\end{array}$ & $\begin{array}{l}B \\
B \\
B \\
B \\
B\end{array}$ & $\begin{array}{l}370 \\
370 \\
370 \\
370 \\
370\end{array}$ & $\begin{array}{l}\cdots \\
\cdots \\
\cdots \\
\cdots\end{array}$ & $\begin{array}{l}\cdots \\
\cdots \\
\cdots \\
\cdots\end{array}$ & $\begin{array}{l}3.8 \mathrm{ML} \\
3.5 \mathrm{ML} \\
3.7 \mathrm{ML} \\
3.6 \mathrm{ML} \\
3.9 \mathrm{ML}\end{array}$ & $\begin{array}{l}\text { GS } \\
\text { GS } \\
\text { GS } \\
\text { GS } \\
\text { GS }\end{array}$ & $\begin{array}{l}\cdots \\
\cdots \\
\cdots \\
\cdots\end{array}$ & $\begin{array}{l}\ldots \\
\ldots \\
\ldots \\
\ldots \\
\ldots\end{array}$ & $\begin{array}{l}\cdots \\
\cdots \\
\cdots \\
\cdots \\
\text { IV }\end{array}$ & 370 \\
\hline $\begin{array}{l}1984 \\
1984 \\
1984 \\
1984 \\
1984\end{array}$ & $\begin{array}{l}\text { NOV } \\
\text { NOV } \\
\text { DEC } \\
\text { DEC } \\
\text { DEC }\end{array}$ & $\begin{array}{l}13 \\
27 \\
08 \\
19 \\
22\end{array}$ & $\begin{array}{l}03 \\
12 \\
09 \\
11 \\
13\end{array}$ & $\begin{array}{l}11 \\
07 \\
41 \\
32 \\
20\end{array}$ & $\begin{array}{l}14.5 \\
50.6 \\
02.0 \\
29.3 \\
27.9\end{array}$ & $\begin{array}{l}44.346 \mathrm{~N} \\
48.871 \mathrm{~N} \\
42.379 \mathrm{~N} \\
42.601 \mathrm{~N} \\
44.241 \mathrm{~N}\end{array}$ & $\begin{array}{l}114.210 W . \\
116.381 W . \\
111.542 W . \\
111.282 W . \\
114.059 W .\end{array}$ & $\begin{array}{l}010 \\
005 \\
005 \\
001 \\
010\end{array}$ & $\begin{array}{l}B \\
C \\
C \\
B \\
C\end{array}$ & $\begin{array}{l}370 \\
370 \\
370 \\
370 \\
370\end{array}$ & $\begin{array}{l}\cdots \\
\cdots \\
\cdots \\
\cdots\end{array}$ & $\begin{array}{l}\cdots \\
\cdots \\
\cdots \\
\cdots\end{array}$ & $\begin{array}{l}3.6 M L \\
2.8 M L \\
2.9 M L \\
2.7 M D \\
3.2 M L\end{array}$ & $\begin{array}{l}\text { GS } \\
G S \\
\text { GS } \\
\text { UU } \\
\text { GS }\end{array}$ & $\begin{array}{l}\cdots \\
\cdots \\
\cdots \\
\cdots\end{array}$ & $\begin{array}{l}\cdots \\
\cdots \\
\cdots \\
\cdots\end{array}$ & $\begin{array}{l}\cdots \\
\cdots \\
\cdots \\
\cdots \\
\cdots\end{array}$ & . \\
\hline $\begin{array}{l}1985 \\
1985 \\
1985 \\
1985 \\
1985\end{array}$ & $\begin{array}{l}\text { JAN } \\
\text { JAN } \\
\text { JAN } \\
\text { JAN } \\
\text { JAN }\end{array}$ & $\begin{array}{l}06 \\
07 \\
07 \\
13 \\
18\end{array}$ & $\begin{array}{l}14 \\
07 \\
07 \\
17 \\
03\end{array}$ & $\begin{array}{l}39 \\
47 \\
47 \\
49 \\
38\end{array}$ & $\begin{array}{l}59.9 \\
02.6 \\
04.4 \\
07.6 \\
07.9\end{array}$ & $\begin{array}{l}44.624 \mathrm{~N} \\
44.554 \mathrm{~N} \\
44.639 \mathrm{~N} \\
44.641 \mathrm{~N} \\
44.703 \mathrm{~N}\end{array}$ & $\begin{array}{l}114.138 W . \\
114.526 W . \\
114.600 W . \\
114.194 W . \\
114.151 W .\end{array}$ & $\begin{array}{l}005 \\
010 \\
025 \\
010 \\
010\end{array}$ & $\begin{array}{l}C \\
C \\
B \\
C \\
B\end{array}$ & $\begin{array}{l}371 \\
371 \\
371 \\
371 \\
371\end{array}$ & $\begin{array}{l}\cdots \\
\cdots \\
\cdots \\
\cdots\end{array}$ & $\begin{array}{l}\cdots \\
\cdots \\
\cdots \\
\cdots\end{array}$ & $\begin{array}{l}3.2 \mathrm{ML} \\
3.2 \mathrm{ML} \\
3.2 \mathrm{ML} \\
3.4 \mathrm{ML} \\
3.7 \mathrm{ML}\end{array}$ & $\begin{array}{l}\text { GS } \\
\text { GS } \\
\text { MMT } \\
\text { GS } \\
\text { GS }\end{array}$ & $\begin{array}{l}\cdots \\
\cdots \\
\cdots \\
\cdots\end{array}$ & $\begin{array}{l}\ldots \\
\ldots \\
\cdots \\
\cdots \\
\cdots\end{array}$ & $\begin{array}{l}\ldots \\
\ldots \\
\ldots \\
\dot{I} I \dot{I}\end{array}$ & $\begin{array}{c}\cdots \\
371\end{array}$ \\
\hline $\begin{array}{l}1985 \\
1985 \\
1985 \\
1985 \\
1985\end{array}$ & $\begin{array}{l}\text { JAN } \\
\text { JAN } \\
\text { JAN } \\
\text { FEB } \\
\text { FEB }\end{array}$ & $\begin{array}{l}20 \\
21 \\
26 \\
04 \\
06\end{array}$ & $\begin{array}{l}01 \\
03 \\
07 \\
04 \\
16\end{array}$ & $\begin{array}{l}18 \\
03 \\
09 \\
33 \\
08\end{array}$ & $\begin{array}{l}24.6 \\
13.2 \\
53.2 \\
32.6 \\
56.0\end{array}$ & $\begin{array}{l}47.526 \mathrm{~N} \\
44.448 \mathrm{~N} \\
43.333 \mathrm{~N} \\
44.442 \mathrm{~N} \\
44.489 \mathrm{~N}\end{array}$ & $\begin{array}{l}115.767 w . \\
114.882 W . \\
111.078 W . \\
114.140 W . \\
114.336 w .\end{array}$ & $\begin{array}{l}001 \\
017 \\
001 \\
007 \\
010\end{array}$ & $\begin{array}{l}B \\
C \\
B \\
B \\
B\end{array}$ & $\begin{array}{l}371 \\
371 \\
371 \\
371 \\
371\end{array}$ & $\begin{array}{l}\cdots \\
\cdots \\
\cdots \\
\cdots\end{array}$ & $\begin{array}{l}\cdots \\
\cdots \\
\cdots \\
\cdots\end{array}$ & $\begin{array}{l}2.8 \mathrm{MD} \\
3.1 \mathrm{ML} \\
3.2 \mathrm{ML} \\
3.2 \mathrm{ML} \\
3.8 \mathrm{ML}\end{array}$ & $\begin{array}{l}\text { MMT } \\
\text { MMT } \\
\text { UU } \\
\text { MMT } \\
\text { GS }\end{array}$ & $\begin{array}{l}\cdots \\
\cdots \\
\cdots \\
\cdots\end{array}$ & $\begin{array}{l}\ldots \\
\ldots \\
\cdots \\
\cdots \\
\cdots\end{array}$ & $\begin{array}{l}\cdots \\
\ldots \\
\cdots \\
i i I\end{array}$ & $\begin{array}{c}\cdots \\
\cdots \\
371\end{array}$ \\
\hline $\begin{array}{l}1985 \\
1985 \\
1985 \\
1985 \\
1985\end{array}$ & $\begin{array}{l}\text { FEB } \\
\text { FEB } \\
\text { FEB } \\
\text { FEB } \\
\text { FEB }\end{array}$ & $\begin{array}{l}06 \\
06 \\
06 \\
07 \\
07\end{array}$ & $\begin{array}{l}18 \\
19 \\
21 \\
02 \\
11\end{array}$ & $\begin{array}{l}13 \\
34 \\
59 \\
14 \\
21\end{array}$ & $\begin{array}{l}54.2 \\
19.4 \\
10.1 \\
04.4 \\
24.7\end{array}$ & $\begin{array}{l}44.355 \mathrm{~N} \\
44.551 \mathrm{~N} \\
44.492 \mathrm{~N} \\
44.421 \mathrm{~N} \\
44.266 \mathrm{~N}\end{array}$ & $\begin{array}{l}114.418 W . \\
114.176 W . \\
114.163 W . \\
114.185 W . \\
114.055 W .\end{array}$ & $\begin{array}{l}010 \\
010 \\
018 \\
010 \\
016\end{array}$ & $\begin{array}{l}\mathrm{C} \\
\mathrm{B} \\
\mathrm{B} \\
\mathrm{B} \\
\mathrm{B}\end{array}$ & $\begin{array}{l}371 \\
371 \\
371 \\
371 \\
371\end{array}$ & $\begin{array}{r}4.7 \\
\ldots \\
\cdots \\
\cdots\end{array}$ & $\begin{array}{l}\cdots \\
\cdots \\
\cdots \\
\cdots\end{array}$ & $\begin{array}{l}3.4 \mathrm{ML} \\
4.8 \mathrm{MD} \\
3.1 \mathrm{ML} \\
3.7 \mathrm{ML} \\
3.2 \mathrm{ML}\end{array}$ & $\begin{array}{l}\text { GS } \\
\text { MMT } \\
\text { MMT } \\
\text { GS } \\
\text { MMT }\end{array}$ & $\begin{array}{l}\cdots \\
\cdots \\
\cdots \\
\cdots\end{array}$ & $\begin{array}{l}\ldots \\
\cdots \\
\cdots \\
\cdots \\
\cdots\end{array}$ & $\begin{array}{l}\mathbf{F} \\
\boldsymbol{V} \\
\cdots \\
\cdots\end{array}$ & $\begin{array}{r}371 \\
371 \\
371 \\
\ldots\end{array}$ \\
\hline $\begin{array}{l}1985 \\
1985 \\
1985 \\
1985 \\
1985\end{array}$ & $\begin{array}{l}\text { FEB } \\
\text { FEB } \\
\text { FEB } \\
\text { FEB } \\
\text { FEB }\end{array}$ & $\begin{array}{l}07 \\
08 \\
08 \\
08 \\
10\end{array}$ & $\begin{array}{l}13 \\
02 \\
05 \\
14 \\
10\end{array}$ & $\begin{array}{l}32 \\
22 \\
36 \\
22 \\
23\end{array}$ & $\begin{array}{l}21.4 \\
20.2 \\
15.1 \\
19.0 \\
45.4\end{array}$ & $\begin{array}{l}44.429 \mathrm{~N} \\
44.465 \mathrm{~N} \\
44.469 \mathrm{~N} \\
47.469 \mathrm{~N} \\
45.228 \mathrm{~N}\end{array}$ & $\begin{array}{l}114.152 W . \\
114.145 W . \\
114.206 W . \\
115.810 W . \\
114.531 W .\end{array}$ & $\begin{array}{l}010 \\
020 \\
020 \\
004 \\
019\end{array}$ & $\begin{array}{l}C \\
B \\
B \\
B \\
C\end{array}$ & $\begin{array}{l}371 \\
371 \\
371 \\
371 \\
371\end{array}$ & $\begin{array}{l}\cdots \\
\cdots \\
\cdots \\
\cdots\end{array}$ & $\begin{array}{l}\ldots \\
\cdots \\
\cdots \\
\cdots\end{array}$ & $\begin{array}{l}3.1 \mathrm{ML} \\
3.2 \mathrm{ML} \\
3.6 \mathrm{ML} \\
2.7 \mathrm{ML} \\
2.5 \mathrm{ML}\end{array}$ & $\begin{array}{l}\text { MMT } \\
\text { MMT } \\
\text { MMT } \\
\text { MMT } \\
\text { MMT }\end{array}$ & $\begin{array}{l}\cdots \\
\cdots \\
\cdots \\
\cdots\end{array}$ & $\begin{array}{l}\ldots \\
\cdots \\
\cdots \\
\cdots \\
\cdots\end{array}$ & $\begin{array}{l}\cdots \\
\cdots \\
\cdots \\
\cdots \\
\cdots\end{array}$ & $\begin{array}{l}\cdots \\
\cdots \\
\cdots \\
\cdots\end{array}$ \\
\hline $\begin{array}{l}1985 \\
1985 \\
1985 \\
1985 \\
1985\end{array}$ & $\begin{array}{l}\text { FEB } \\
\text { FEB } \\
\text { FEB } \\
\text { FEB } \\
\text { FEB }\end{array}$ & $\begin{array}{l}11 \\
11 \\
12 \\
15 \\
17\end{array}$ & $\begin{array}{l}13 \\
16 \\
04 \\
09 \\
05\end{array}$ & $\begin{array}{l}31 \\
07 \\
14 \\
58 \\
31\end{array}$ & $\begin{array}{l}50.0 \\
03.8 \\
48.7 \\
14.4 \\
28.6\end{array}$ & $\begin{array}{l}44.369 \mathrm{~N} \\
44.457 \mathrm{~N} \\
44.856 \mathrm{~N} \\
44.126 \mathrm{~N} \\
44.415 \mathrm{~N}\end{array}$ & $\begin{array}{l}114.493 w . \\
114.233 w . \\
113.410 W . \\
114.355 W . \\
114.080 W .\end{array}$ & $\begin{array}{l}005 \\
010 \\
005 \\
023 \\
019\end{array}$ & $\begin{array}{l}\mathrm{C} \\
\mathrm{B} \\
\mathrm{B} \\
\mathrm{C} \\
\mathrm{C}\end{array}$ & $\begin{array}{l}371 \\
371 \\
371 \\
371 \\
371\end{array}$ & $\begin{array}{l}\cdots \\
\cdots \\
\cdots \\
\cdots\end{array}$ & $\begin{array}{l}\cdots \\
\cdots \\
\cdots \\
\cdots\end{array}$ & $\begin{array}{l}\text { 3. } 9 \mathrm{ML} \\
3.8 \mathrm{ML} \\
3.2 \mathrm{ML} \\
3.0 \mathrm{ML} \\
2.7 \mathrm{ML}\end{array}$ & $\begin{array}{l}\text { GS } \\
\text { GS } \\
\text { MMT } \\
\text { MMT } \\
\text { MMT }\end{array}$ & $\begin{array}{l}\cdots \\
\cdots \\
\cdots \\
\cdots\end{array}$ & $\begin{array}{l}\ldots \\
\cdots \\
\cdots \\
\cdots\end{array}$ & $\begin{array}{l}\cdots \\
F \\
\ldots \\
\cdots\end{array}$ & $\begin{array}{r}3 \ddot{1} \\
\ldots \\
\cdots\end{array}$ \\
\hline $\begin{array}{l}1985 \\
1985 \\
1985 \\
1985 \\
1985\end{array}$ & $\begin{array}{l}\text { FEB } \\
\text { FEB } \\
\text { FEB } \\
\text { FEB } \\
\text { FEB }\end{array}$ & $\begin{array}{l}19 \\
24 \\
24 \\
25 \\
27\end{array}$ & $\begin{array}{l}19 \\
05 \\
22 \\
11 \\
07\end{array}$ & $\begin{array}{l}25 \\
37 \\
00 \\
46 \\
29\end{array}$ & $\begin{array}{l}49.2 \\
13.2 \\
38.1 \\
06.5 \\
07.8\end{array}$ & $\begin{array}{l}44.469 \mathrm{~N} \\
42.464 \mathrm{~N} \\
44.475 \mathrm{~N} \\
44.501 \mathrm{~N} \\
44.480 \mathrm{~N}\end{array}$ & $\begin{array}{l}113.378 W . \\
111.822 W . \\
114.185 W . \\
114.190 W . \\
114.187 W .\end{array}$ & $\begin{array}{l}005 \\
007 \\
015 \\
010 \\
010\end{array}$ & $\begin{array}{l}C \\
B \\
C \\
C \\
C\end{array}$ & $\begin{array}{l}371 \\
371 \\
371 \\
371 \\
371\end{array}$ & $\begin{array}{l}\cdots \\
\cdots \\
\cdots \\
\cdots\end{array}$ & $\begin{array}{l}\ldots \\
\cdots \\
\ldots \\
\ldots\end{array}$ & $\begin{array}{l}3.0 \mathrm{ML} \\
2.7 \mathrm{ML} \\
2.9 \mathrm{ML} \\
3.3 \mathrm{ML} \\
3.1 \mathrm{ML}\end{array}$ & $\begin{array}{l}\text { MMT } \\
\text { UU } \\
\text { MMT } \\
\text { GS } \\
\text { GS }\end{array}$ & $\begin{array}{l}\cdots \\
\cdots \\
\cdots \\
\cdots\end{array}$ & $\begin{array}{l}\ldots \\
\cdots \\
\cdots \\
\ldots\end{array}$ & $\begin{array}{l}\cdots \\
\cdots \\
\text { II } \\
\ldots\end{array}$ & $\begin{array}{c}\cdots \\
\cdots \\
371 \\
\ldots\end{array}$ \\
\hline
\end{tabular}




\begin{tabular}{|c|c|c|c|c|c|c|c|c|c|c|c|c|c|c|c|c|c|c|}
\hline $\begin{array}{l}1985 \\
1985 \\
1985 \\
1985 \\
1985\end{array}$ & $\begin{array}{l}\text { MAR } \\
\text { MAR } \\
\text { MAR } \\
\text { MAR } \\
\text { MAR }\end{array}$ & $\begin{array}{l}03 \\
11 \\
17 \\
21 \\
22\end{array}$ & $\begin{array}{l}09 \\
19 \\
06 \\
09 \\
18\end{array}$ & $\begin{array}{l}44 \\
47 \\
56 \\
02 \\
07\end{array}$ & $\begin{array}{l}05.8 \\
26.5 \\
17.1 \\
57.3 \\
55.0\end{array}$ & $\begin{array}{l}44.243 \mathrm{~N} . \\
43.994 \mathrm{~N} \\
44.553 \mathrm{~N} \\
44.286 \mathrm{~N} . \\
44.264 \mathrm{~N}\end{array}$ & $\begin{array}{l}113.996 W . \\
114.384 W . \\
114.182 W . \\
114.076 W . \\
114.042 W .\end{array}$ & $\begin{array}{l}018 \\
022 \\
010 \\
013 \\
017\end{array}$ & $\begin{array}{l}C \\
B \\
B \\
B \\
C\end{array}$ & $\begin{array}{l}371 \\
371 \\
371 \\
371 \\
371\end{array}$ & 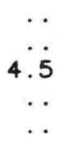 & $\begin{array}{l}\cdots \\
\cdots \\
\cdots \\
\cdots\end{array}$ & $\begin{array}{l}\text { 3. 3ML } \\
3.0 \mathrm{ML} \\
4.7 \mathrm{MD} \\
2.8 \mathrm{ML} \\
3.1 \mathrm{ML}\end{array}$ & $\begin{array}{l}\text { MMT } \\
\text { MMT } \\
\text { MMT } \\
\text { MMT } \\
\text { MMT }\end{array}$ & $\begin{array}{l}\cdots \\
\cdots \\
\cdots \\
\cdots\end{array}$ & $\begin{array}{l}\cdots \\
\cdots \\
\cdots \\
\cdots \\
\cdots\end{array}$ & $\begin{array}{l}\cdots \\
\cdots \\
\cdots \\
\cdots\end{array}$ & $\begin{array}{r}\cdots \\
371 \\
\cdots \\
\cdots\end{array}$ \\
\hline $\begin{array}{l}1985 \\
1985 \\
1985 \\
1985 \\
1985\end{array}$ & $\begin{array}{l}\text { MAR } \\
\text { MAR } \\
\text { MAR } \\
\text { MAR } \\
\text { MAR }\end{array}$ & $\begin{array}{l}23 \\
23 \\
25 \\
31 \\
31\end{array}$ & $\begin{array}{l}18 \\
18 \\
07 \\
00 \\
16\end{array}$ & $\begin{array}{l}03 \\
10 \\
55 \\
26 \\
05\end{array}$ & $\begin{array}{l}53.9 \\
19.4 \\
12.3 \\
31.4 \\
34.1\end{array}$ & $\begin{array}{l}44.358 \mathrm{~N} . \\
44.304 \mathrm{~N} \\
44.465 \mathrm{~N} \\
44.843 \mathrm{~N} \\
44.125 \mathrm{~N} .\end{array}$ & $\begin{array}{l}113.777 w . \\
113.796 w . \\
114.153 w . \\
114.266 w . \\
113.851 w .\end{array}$ & $\begin{array}{l}005 \\
005 \\
015 \\
018 \\
005\end{array}$ & $\begin{array}{l}C \\
C \\
B \\
C \\
B\end{array}$ & $\begin{array}{l}371 \\
371 \\
371 \\
371 \\
371\end{array}$ & $\begin{array}{l}\cdots \\
\cdots \\
\cdots \\
\cdots\end{array}$ & $\begin{array}{l}\cdots \\
\cdots \\
\cdots \\
\cdots\end{array}$ & $\begin{array}{l}3.5 \mathrm{ML} \\
3.4 \mathrm{ML} \\
3.2 \mathrm{ML} \\
2.5 \mathrm{ML} \\
3.1 \mathrm{ML}\end{array}$ & $\begin{array}{l}\text { GS } \\
\text { GS } \\
\text { MMT } \\
\text { MMT } \\
\text { MMT }\end{array}$ & $\begin{array}{l}\cdots \\
\cdots \\
\cdots \\
\cdots\end{array}$ & $\begin{array}{l}\cdots \\
\cdots \\
\cdots \\
\cdots \\
\cdots\end{array}$ & $\begin{array}{l}\cdots \\
\cdots \\
\cdots \\
\cdots \\
\cdots\end{array}$ & $\begin{array}{l}\cdots \\
\cdots \\
\cdots \\
\cdots\end{array}$ \\
\hline $\begin{array}{l}1985 \\
1985 \\
1985 \\
1985 \\
1985\end{array}$ & $\begin{array}{l}\text { APR } \\
\text { APR } \\
\text { APR } \\
\text { APR } \\
\text { APR }\end{array}$ & $\begin{array}{l}01 \\
05 \\
10 \\
11 \\
11\end{array}$ & $\begin{array}{l}07 \\
05 \\
03 \\
08 \\
08\end{array}$ & $\begin{array}{l}08 \\
43 \\
02 \\
22 \\
43\end{array}$ & $\begin{array}{l}49.0 \\
26.8 \\
02.9 \\
02.3 \\
57.7\end{array}$ & $\begin{array}{l}44.425 \mathrm{~N} \\
42.390 \mathrm{~N} \\
44.304 \mathrm{~N} \\
44.745 \mathrm{~N} \\
44.749 \mathrm{~N}\end{array}$ & $\begin{array}{l}114.083 W . \\
111.571 W . \\
114.228 W . \\
114.508 W . \\
114.538 W .\end{array}$ & $\begin{array}{l}015 \\
005 \\
010 \\
019 \\
014\end{array}$ & $\begin{array}{l}B \\
B \\
B \\
B \\
C\end{array}$ & $\begin{array}{l}371 \\
371 \\
371 \\
371 \\
371\end{array}$ & $\begin{array}{l}\cdots \\
\cdots \\
\cdots \\
\cdots\end{array}$ & $\begin{array}{l}\cdots \\
\cdots \\
\cdots \\
\cdots\end{array}$ & $\begin{array}{l}2.8 \mathrm{ML} \\
3.2 \mathrm{ML} \\
3.2 \mathrm{ML} \\
2.8 \mathrm{ML} \\
2.7 \mathrm{ML}\end{array}$ & $\begin{array}{l}\text { MMT } \\
\text { UU } \\
\text { GS } \\
\text { MMT } \\
\text { MMT }\end{array}$ & $\begin{array}{l}\cdots \\
\cdots \\
\cdots \\
\cdots\end{array}$ & $\begin{array}{l}\cdots \\
\cdots \\
\cdots \\
\cdots \\
\cdots\end{array}$ & $\begin{array}{l}\cdots \\
\cdots \\
\cdots \\
\cdots \\
\cdots\end{array}$ & $\begin{array}{l}\cdots \\
\cdots \\
\cdots \\
\cdots\end{array}$ \\
\hline $\begin{array}{l}1985 \\
1985 \\
1985 \\
1985 \\
1985\end{array}$ & $\begin{array}{l}\text { APR } \\
\text { APR } \\
\text { APR } \\
\text { APR } \\
\text { APR }\end{array}$ & $\begin{array}{l}12 \\
13 \\
14 \\
17 \\
18\end{array}$ & $\begin{array}{l}20 \\
11 \\
21 \\
10 \\
06\end{array}$ & $\begin{array}{l}57 \\
42 \\
39 \\
29 \\
22\end{array}$ & $\begin{array}{l}51.3 \\
17.5 \\
10.0 \\
51.4 \\
18.3\end{array}$ & $\begin{array}{l}44.442 \mathrm{~N} \\
44.493 \mathrm{~N} \\
43.961 \mathrm{~N} \\
44.192 \mathrm{~N} \\
44.448 \mathrm{~N}\end{array}$ & $\begin{array}{l}114.067 \mathrm{~W} . \\
113.628 \mathrm{w} . \\
113.733 \mathrm{w} . \\
113.488 \mathrm{w} \text {. } \\
114.129 \mathrm{w} .\end{array}$ & $\begin{array}{l}021 \\
010 \\
015 \\
010 \\
009\end{array}$ & $\begin{array}{l}B \\
C \\
B \\
C \\
B\end{array}$ & $\begin{array}{l}371 \\
371 \\
371 \\
371 \\
371\end{array}$ & $\begin{array}{l}\cdots \\
\cdots \\
\cdots \\
\cdots\end{array}$ & $\begin{array}{l}\cdots \\
\cdots \\
\cdots \\
\cdots\end{array}$ & $\begin{array}{l}\text { 3. } 1 \mathrm{ML} \\
2.5 \mathrm{ML} \\
3.0 \mathrm{ML} \\
3.0 \mathrm{ML} \\
3.2 \mathrm{ML}\end{array}$ & $\begin{array}{l}\text { MMT } \\
\text { MMT } \\
\text { GS } \\
\text { GS } \\
\text { MMT }\end{array}$ & $\begin{array}{l}\cdots \\
\cdots \\
\cdots \\
\cdots\end{array}$ & $\begin{array}{l}\cdots \\
\cdots \\
\cdots \\
\cdots \\
\cdots\end{array}$ & $\begin{array}{l}\cdots \\
\cdots \\
\cdots \\
\cdots \\
\cdots\end{array}$ & $\begin{array}{l}\cdots \\
\cdots \\
\cdots \\
\cdots\end{array}$ \\
\hline $\begin{array}{l}1985 \\
1985 \\
1985 \\
1985 \\
1985\end{array}$ & $\begin{array}{l}\text { APR } \\
\text { APR } \\
\text { APR } \\
\text { MAY } \\
\text { MAY }\end{array}$ & $\begin{array}{l}19 \\
19 \\
20 \\
06 \\
09\end{array}$ & $\begin{array}{l}00 \\
10 \\
06 \\
23 \\
15\end{array}$ & $\begin{array}{l}03 \\
54 \\
20 \\
34 \\
29\end{array}$ & $\begin{array}{l}49.3 \\
14.3 \\
34.3 \\
19.6 \\
37.6\end{array}$ & $\begin{array}{l}44.500 \mathrm{~N} \\
44.229 \mathrm{~N} \\
44.474 \mathrm{~N} \\
44.467 \mathrm{~N} \\
44.047 \mathrm{~N}\end{array}$ & $\begin{array}{l}114.095 W . \\
114.005 W . \\
114.273 W . \\
114.275 W . \\
114.377 W .\end{array}$ & $\begin{array}{l}006 \\
010 \\
010 \\
005 \\
005\end{array}$ & $\begin{array}{l}B \\
B \\
B \\
C \\
C\end{array}$ & $\begin{array}{l}371 \\
371 \\
371 \\
371 \\
371\end{array}$ & $\begin{array}{l}\cdots \\
\cdots \\
\cdots \\
\cdots\end{array}$ & $\begin{array}{l}\cdots \\
\cdots \\
\cdots \\
\cdots\end{array}$ & $\begin{array}{l}3.0 \mathrm{ML} \\
2.8 \mathrm{ML} \\
3.3 \mathrm{ML} \\
3.2 \mathrm{ML} \\
3.0 \mathrm{ML}\end{array}$ & $\begin{array}{l}\text { MMT } \\
\text { MMT } \\
\text { GS } \\
\text { MMT } \\
\text { GS }\end{array}$ & $\begin{array}{l}\cdots \\
\cdots \\
\cdots \\
\cdots\end{array}$ & $\begin{array}{l}\cdots \\
\cdots \\
\cdots \\
\cdots \\
\cdots\end{array}$ & $\begin{array}{l}\cdots \\
\cdots \\
\cdots \\
\cdots \\
\cdots\end{array}$ & $\begin{array}{l}\cdots \\
\cdots \\
\cdots \\
\cdots\end{array}$ \\
\hline $\begin{array}{l}1985 \\
1985 \\
1985 \\
1985 \\
1985\end{array}$ & $\begin{array}{l}\text { MAY } \\
\text { MAY } \\
\text { MAY } \\
\text { MAY } \\
\text { MAY }\end{array}$ & $\begin{array}{l}10 \\
10 \\
13 \\
13 \\
16\end{array}$ & $\begin{array}{l}18 \\
22 \\
08 \\
09 \\
05\end{array}$ & $\begin{array}{l}16 \\
00 \\
23 \\
36 \\
08\end{array}$ & $\begin{array}{l}36.7 \\
58.4 \\
35.2 \\
36.2 \\
35.9\end{array}$ & $\begin{array}{l}44.475 \mathrm{~N} \\
44.599 \mathrm{~N} \\
44.045 \mathrm{~N} \\
44.361 \mathrm{~N} \\
44.716 \mathrm{~N}\end{array}$ & $\begin{array}{l}114.418 W . \\
114.116 W . \\
113.874 w . \\
114.476 w . \\
114.309 w .\end{array}$ & $\begin{array}{l}010 \\
005 \\
005 \\
005 \\
008\end{array}$ & $\begin{array}{l}B \\
C \\
B \\
C \\
B\end{array}$ & $\begin{array}{l}371 \\
371 \\
371 \\
371 \\
371\end{array}$ & $\begin{array}{l}\cdots \\
\cdots \\
\cdots \\
\cdots\end{array}$ & $\begin{array}{l}\cdots \\
\cdots \\
\cdots \\
\cdots\end{array}$ & $\begin{array}{l}2.9 \mathrm{ML} \\
3.1 \mathrm{ML} \\
3.2 \mathrm{ML} \\
3.0 \mathrm{ML} \\
2.6 \mathrm{ML}\end{array}$ & $\begin{array}{l}\text { MMT } \\
\text { MMT } \\
\text { GS } \\
\text { MMT } \\
\text { MMT }\end{array}$ & $\begin{array}{l}\cdots \\
\cdots \\
\cdots \\
\cdots\end{array}$ & $\begin{array}{l}\cdots \\
\cdots \\
\cdots \\
\cdots \\
\cdots\end{array}$ & $\begin{array}{l}\cdots \\
\cdots \\
\cdots \\
\cdots \\
\cdots\end{array}$ & $\begin{array}{l}\cdots \\
\ldots \\
\cdots \\
\cdots\end{array}$ \\
\hline $\begin{array}{l}1985 \\
1985 \\
1985 \\
1985 \\
1985\end{array}$ & $\begin{array}{l}\text { JUN } \\
\text { JUN } \\
\text { JUN } \\
\text { JUN } \\
\text { JUN }\end{array}$ & $\begin{array}{l}05 \\
07 \\
08 \\
11 \\
14\end{array}$ & $\begin{array}{l}02 \\
09 \\
13 \\
23 \\
21\end{array}$ & $\begin{array}{l}15 \\
55 \\
08 \\
09 \\
41\end{array}$ & $\begin{array}{l}07.5 \\
20.9 \\
23.2 \\
37.1 \\
52.6\end{array}$ & $\begin{array}{l}47.646 \mathrm{~N} \\
44.258 \mathrm{~N} \\
44.255 \mathrm{~N} \\
44.499 \mathrm{~N} \\
44.924 \mathrm{~N}\end{array}$ & $\begin{array}{l}115.607 w . \\
114.038 w . \\
114.199 w . \\
114.216 w . \\
114.278 w .\end{array}$ & $\begin{array}{l}003 \\
014 \\
010 \\
005 \\
011\end{array}$ & $\begin{array}{l}B \\
B \\
C \\
C \\
C\end{array}$ & $\begin{array}{l}371 \\
371 \\
371 \\
371 \\
371\end{array}$ & $\begin{array}{l}\cdots \\
\cdots \\
\cdots \\
\cdots\end{array}$ & $\begin{array}{l}\cdots \\
\cdots \\
\cdots \\
\cdots\end{array}$ & $\begin{array}{l}2.9 \mathrm{ML} \\
2.9 \mathrm{ML} \\
3.4 \mathrm{ML} \\
3.9 \mathrm{ML} \\
2.8 \mathrm{ML}\end{array}$ & $\begin{array}{l}\text { MMT } \\
\text { MMT } \\
\text { GS } \\
\text { GS } \\
\text { MMT }\end{array}$ & $\begin{array}{l}\cdots \\
\cdots \\
\cdots \\
\cdots\end{array}$ & $\begin{array}{l}\cdots \\
\cdots \\
\cdots \\
\cdots \\
\cdots\end{array}$ & $\begin{array}{l}\cdots \\
\cdots \\
\cdots \\
\cdots \\
\cdots\end{array}$ & $\begin{array}{l}\cdots \\
\cdots \\
\cdots \\
\cdots\end{array}$ \\
\hline $\begin{array}{l}1985 \\
1985 \\
1985 \\
1985 \\
1985\end{array}$ & $\begin{array}{l}\text { JUN } \\
\text { JUN } \\
\text { JUN } \\
\text { JUN } \\
\text { JUN }\end{array}$ & $\begin{array}{l}15 \\
15 \\
19 \\
19 \\
23\end{array}$ & $\begin{array}{l}01 \\
05 \\
01 \\
05 \\
08\end{array}$ & $\begin{array}{l}19 \\
23 \\
13 \\
18 \\
10\end{array}$ & $\begin{array}{l}35.7 \\
30.7 \\
20.2 \\
44.7 \\
55.8\end{array}$ & $\begin{array}{l}43.507 \mathrm{~N} . \\
43.506 \mathrm{~N} . \\
46.048 \mathrm{~N} . \\
44.840 \mathrm{~N} . \\
44.668 \mathrm{~N} .\end{array}$ & $\begin{array}{l}113.622 W . \\
113.618 w . \\
115.736 w . \\
114.277 w . \\
113.843 w .\end{array}$ & $\begin{array}{l}012 \\
019 \\
008 \\
014 \\
018\end{array}$ & $\begin{array}{l}c \\
C \\
C \\
c \\
C\end{array}$ & $\begin{array}{l}371 \\
371 \\
371 \\
371 \\
371\end{array}$ & $\begin{array}{l}\cdots \\
\cdots \\
\cdots \\
\cdots\end{array}$ & $\begin{array}{l}\cdots \\
\cdots \\
\cdots \\
\cdots\end{array}$ & $\begin{array}{l}\text { 3. } 4 M L \\
3.2 \mathrm{ML} \\
2.5 \mathrm{ML} \\
2.6 \mathrm{ML} \\
2.6 \mathrm{ML}\end{array}$ & $\begin{array}{l}\text { MMT } \\
\text { MMT } \\
\text { MMT } \\
\text { MMT } \\
\text { MMT }\end{array}$ & $\begin{array}{l}\cdots \\
\cdots \\
\cdots \\
\cdots\end{array}$ & $\begin{array}{l}\cdots \\
\cdots \\
\cdots \\
\cdots \\
\cdots\end{array}$ & $\begin{array}{l}\cdots \\
\cdots \\
\cdots \\
\cdots\end{array}$ & $\begin{array}{l}\ldots \\
\cdots \\
\cdots \\
\cdots\end{array}$ \\
\hline $\begin{array}{l}1985 \\
1985 \\
1985 \\
1985 \\
1985\end{array}$ & $\begin{array}{l}\text { JUN } \\
\text { JUN } \\
\text { JUN } \\
\text { JUN } \\
\text { JUL }\end{array}$ & $\begin{array}{l}23 \\
23 \\
27 \\
29 \\
02\end{array}$ & $\begin{array}{l}10 \\
16 \\
11 \\
11 \\
03\end{array}$ & $\begin{array}{l}43 \\
41 \\
11 \\
12 \\
03\end{array}$ & $\begin{array}{l}38.3 \\
04.5 \\
07.8 \\
21.4 \\
56.0\end{array}$ & $\begin{array}{l}45.542 \mathrm{~N} . \\
44.799 \mathrm{~N} . \\
44.117 \mathrm{~N} . \\
44.419 \mathrm{~N} . \\
43.255 \mathrm{~N} .\end{array}$ & $\begin{array}{l}115.909 W . \\
114.387 W . \\
114.382 W . \\
113.917 W . \\
111.154 W .\end{array}$ & $\begin{array}{l}035 \\
020 \\
005 \\
001 \\
005\end{array}$ & $\begin{array}{l}D \\
C \\
C \\
C \\
B\end{array}$ & $\begin{array}{l}371 \\
371 \\
371 \\
371 \\
371\end{array}$ & $\begin{array}{l}\cdots \\
\cdots \\
\cdots \\
\cdots\end{array}$ & $\begin{array}{l}\cdots \\
\cdots \\
\cdots \\
\cdots\end{array}$ & $\begin{array}{l}\text { 2. } 8 \mathrm{ML} \\
\text { 2. } 6 \mathrm{ML} \\
\text { 3. } \mathrm{OML} \\
\text { 2. } 7 \mathrm{ML} \\
\text { 4. } \mathrm{OML}\end{array}$ & $\begin{array}{l}\text { MMT } \\
\text { MMT } \\
\text { GS } \\
\text { MMT } \\
\text { GS }\end{array}$ & $\begin{array}{l}\cdots \\
\cdots \\
\cdots \\
\cdots\end{array}$ & $\begin{array}{l}\cdots \\
\cdots \\
\cdots \\
\cdots \\
\cdots\end{array}$ & $\begin{array}{l}\cdots \\
\cdots \\
\cdots \\
\text { IV }\end{array}$ & $\begin{array}{c}\cdots \\
\cdots \\
3 \ddot{j}\end{array}$ \\
\hline $\begin{array}{l}1985 \\
1985 \\
1985 \\
1985 \\
1985\end{array}$ & $\begin{array}{l}\text { JUL } \\
\text { JUL } \\
\text { JUL } \\
\text { JUL } \\
\text { JUL }\end{array}$ & $\begin{array}{l}08 \\
16 \\
16 \\
17 \\
17\end{array}$ & $\begin{array}{l}14 \\
18 \\
19 \\
00 \\
00\end{array}$ & $\begin{array}{l}30 \\
43 \\
13 \\
01 \\
16\end{array}$ & $\begin{array}{l}43.4 \\
07.3 \\
28.4 \\
53.7 \\
55.0\end{array}$ & $\begin{array}{l}44.437 \mathrm{~N} \\
44.034 \mathrm{~N} \\
43.924 \mathrm{~N} \\
45.076 \mathrm{~N} . \\
45.046 \mathrm{~N} .\end{array}$ & $\begin{array}{l}113.992 W . \\
114.723 W . \\
114.671 W . \\
114.354 W . \\
114.349 W .\end{array}$ & $\begin{array}{l}005 \\
005 \\
003 \\
005 \\
005\end{array}$ & $\begin{array}{l}C \\
C \\
C \\
B \\
B\end{array}$ & $\begin{array}{l}371 \\
371 \\
371 \\
371 \\
371\end{array}$ & $\begin{array}{l}\cdots \\
\cdots \\
\cdots \\
\cdots\end{array}$ & $\begin{array}{l}\cdots \\
\cdots \\
\cdots \\
\cdots\end{array}$ & $\begin{array}{l}\text { 3. } 3 \mathrm{ML} \\
\text { 3. } 8 \mathrm{ML} \\
\text { 2. } 8 \mathrm{ML} \\
\text { 3. } 4 \mathrm{ML} \\
\text { 3. } 6 \mathrm{ML}\end{array}$ & $\begin{array}{l}\text { GS } \\
\text { GS } \\
\text { GS } \\
\text { GS } \\
\text { GS }\end{array}$ & $\begin{array}{l}\cdots \\
\cdots \\
\cdots \\
\cdots\end{array}$ & $\begin{array}{l}\cdots \\
\cdots \\
\cdots \\
\cdots \\
\cdots\end{array}$ & $\begin{array}{l}\cdots \\
\cdots \\
\cdots \\
\cdots \\
\cdots\end{array}$ & $\begin{array}{l}\ldots \\
\cdots \\
\cdots \\
\cdots\end{array}$ \\
\hline $\begin{array}{l}1985 \\
1985 \\
1985 \\
1985 \\
1985\end{array}$ & $\begin{array}{l}\text { JUL } \\
\text { JUL } \\
\text { JUL } \\
\text { JUL } \\
\text { AUG }\end{array}$ & $\begin{array}{l}17 \\
19 \\
20 \\
28 \\
01\end{array}$ & $\begin{array}{l}00 \\
12 \\
17 \\
06 \\
11\end{array}$ & $\begin{array}{l}20 \\
52 \\
33 \\
13 \\
50\end{array}$ & $\begin{array}{l}13.9 \\
38.8 \\
22.4 \\
47.8 \\
54.7\end{array}$ & $\begin{array}{l}45.038 \mathrm{~N} . \\
44.011 \mathrm{~N} . \\
44.482 \mathrm{~N} . \\
44.305 \mathrm{~N} . \\
44.594 \mathrm{~N} .\end{array}$ & $\begin{array}{l}114.369 w . \\
114.833 w \text {. } \\
114.097 w . \\
114.147 w . \\
114.629 W .\end{array}$ & $\begin{array}{l}020 \\
005 \\
005 \\
005 \\
024\end{array}$ & $\begin{array}{l}\mathrm{C} \\
\mathrm{B} \\
\mathrm{B} \\
\mathrm{C} \\
\mathrm{C}\end{array}$ & $\begin{array}{l}371 \\
371 \\
371 \\
371 \\
371\end{array}$ & $\begin{array}{l}\cdots \\
\cdots \\
\cdots \\
\cdots\end{array}$ & $\begin{array}{l}\cdots \\
\cdots \\
\cdots \\
\cdots\end{array}$ & $\begin{array}{l}2.6 \mathrm{ML} \\
3.5 \mathrm{ML} \\
3.5 \mathrm{ML} \\
3.1 \mathrm{ML} \\
2.8 \mathrm{ML}\end{array}$ & $\begin{array}{l}\text { MMT } \\
\text { GS } \\
\text { GS } \\
\text { GS } \\
\text { MMT }\end{array}$ & $\begin{array}{l}\cdots \\
\cdots \\
\cdots \\
\cdots\end{array}$ & $\begin{array}{l}\cdots \\
\cdots \\
\cdots \\
\cdots \\
\cdots\end{array}$ & $\begin{array}{l}\cdots \\
\cdots \\
\cdots \\
\cdots \\
\cdots\end{array}$ & $\begin{array}{l}\cdots \\
\cdots \\
\cdots \\
\cdots\end{array}$ \\
\hline $\begin{array}{l}1985 \\
1985 \\
1985\end{array}$ & $\begin{array}{l}\text { AUG } \\
\text { AUG } \\
\text { AUG }\end{array}$ & $\begin{array}{l}03 \\
05 \\
06\end{array}$ & $\begin{array}{l}11 \\
11 \\
19\end{array}$ & $\begin{array}{l}20 \\
06 \\
32\end{array}$ & $\begin{array}{l}58.3 \\
52.9 \\
00.8\end{array}$ & $\begin{array}{l}44.422 \mathrm{~N} \\
43.821 \mathrm{~N} \\
44.057 \mathrm{~N}\end{array}$ & $\begin{array}{l}114.232 W . \\
113.648 W . \\
114.195 W .\end{array}$ & $\begin{array}{l}021 \\
027 \\
005\end{array}$ & $\begin{array}{l}C \\
C \\
C\end{array}$ & $\begin{array}{l}371 \\
371 \\
371\end{array}$ & $\begin{array}{l}\cdots \\
\cdots \\
\cdots\end{array}$ & $\cdots$ & $\begin{array}{l}2.6 \mathrm{ML} \\
2.8 \mathrm{ML} \\
2.8 \mathrm{ML}\end{array}$ & $\begin{array}{l}\text { MMT } \\
\text { MMT } \\
\text { GS }\end{array}$ & $\begin{array}{l}\cdots \\
\cdots \\
\cdots\end{array}$ & $\begin{array}{l}\cdots \\
\cdots \\
\cdots\end{array}$ & $\begin{array}{l}\cdots \\
\cdots \\
\cdots\end{array}$ & $\begin{array}{l}\cdots \\
\cdots\end{array}$ \\
\hline
\end{tabular}




\begin{tabular}{|c|c|c|c|c|c|c|c|c|c|c|c|c|c|c|c|c|c|c|}
\hline $\begin{array}{l}1985 \\
1985\end{array}$ & $\begin{array}{l}\text { AUG } \\
\text { AUG }\end{array}$ & $\begin{array}{l}07 \\
07\end{array}$ & $\begin{array}{l}07 \\
11\end{array}$ & $\begin{array}{l}10 \\
23\end{array}$ & $\begin{array}{l}33.2 \\
18.3\end{array}$ & $\begin{array}{l}42.108 \mathrm{~N} \\
44.255 \mathrm{~N}\end{array}$ & $\begin{array}{l}112.322 W . \\
114.034 W .\end{array}$ & $\begin{array}{l}001 \\
021\end{array}$ & $\begin{array}{l}B \\
C\end{array}$ & $\begin{array}{l}371 \\
371\end{array}$ & $\begin{array}{l}\cdots \\
\cdots\end{array}$ & $\begin{array}{l}\cdots \\
\cdots\end{array}$ & $\begin{array}{l}2.8 \mathrm{ML} \\
2.6 \mathrm{ML}\end{array}$ & $\begin{array}{l}\text { UU } \\
\text { MMT }\end{array}$ & $\begin{array}{l}\cdots \\
\cdots\end{array}$ & $\begin{array}{l}\cdots \\
\cdots\end{array}$ & $\begin{array}{l}\text { I I I } \\
\ldots\end{array}$ & $\begin{array}{r}371 \\
\ldots\end{array}$ \\
\hline $\begin{array}{l}1985 \\
1985 \\
1985 \\
1985 \\
1985\end{array}$ & $\begin{array}{l}\text { AUG } \\
\text { AUG } \\
\text { AUG } \\
\text { AUG } \\
\text { AUG }\end{array}$ & $\begin{array}{l}09 \\
13 \\
13 \\
13 \\
14\end{array}$ & $\begin{array}{l}10 \\
01 \\
01 \\
01 \\
06\end{array}$ & $\begin{array}{l}10 \\
03 \\
05 \\
18 \\
58\end{array}$ & $\begin{array}{l}51.7 \\
10.9 \\
24.1 \\
20.9 \\
42.6\end{array}$ & $\begin{array}{l}46.927 \mathrm{~N} \\
44.532 \mathrm{~N} \\
44.510 \mathrm{~N} \\
44.512 \mathrm{~N} \\
44.423 \mathrm{~N}\end{array}$ & $\begin{array}{l}116.755 w . \\
114.462 W . \\
114.411 w . \\
114.471 w . \\
114.288 w .\end{array}$ & $\begin{array}{l}027 \\
014 \\
011 \\
022 \\
005\end{array}$ & $\begin{array}{l}C \\
C \\
C \\
C \\
C\end{array}$ & $\begin{array}{l}371 \\
371 \\
371 \\
371 \\
371\end{array}$ & $\begin{array}{l}\cdots \\
\cdots \\
\cdots \\
\cdots\end{array}$ & $\begin{array}{l}\cdots \\
\cdots \\
\cdots \\
\cdots\end{array}$ & $\begin{array}{l}2.9 M L \\
2.7 M L \\
2.7 M L \\
2.5 M L \\
2.7 M L\end{array}$ & $\begin{array}{l}\text { MMT } \\
\text { MMT } \\
\text { MMT } \\
\text { MMT } \\
\text { GS }\end{array}$ & $\begin{array}{l}\cdots \\
\cdots \\
\cdots \\
\cdots\end{array}$ & $\begin{array}{l}\cdots \\
\cdots \\
\cdots \\
\cdots \\
\cdots\end{array}$ & $\begin{array}{l}\cdots \\
\cdots \\
\cdots \\
\cdots \\
\cdots\end{array}$ & $\begin{array}{l}\cdots \\
\cdots \\
\cdots \\
\cdots\end{array}$ \\
\hline $\begin{array}{l}1985 \\
1985 \\
1985 \\
1985 \\
1985\end{array}$ & 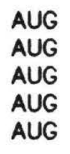 & $\begin{array}{l}15 \\
23 \\
24 \\
28 \\
28\end{array}$ & $\begin{array}{l}07 \\
05 \\
19 \\
00 \\
15\end{array}$ & $\begin{array}{l}41 \\
12 \\
07 \\
24 \\
08\end{array}$ & $\begin{array}{l}44.5 \\
08.2 \\
58.2 \\
06.2 \\
24.6\end{array}$ & $\begin{array}{l}44.265 \mathrm{~N} \\
44.483 \mathrm{~N} \\
46.880 \mathrm{~N} \\
44.587 \mathrm{~N} \\
44.412 \mathrm{~N}\end{array}$ & $\begin{array}{l}114.112 W . \\
114.165 W . \\
116.768 W . \\
114.401 W . \\
114.413 W .\end{array}$ & $\begin{array}{l}005 \\
005 \\
005 \\
003 \\
005\end{array}$ & $\begin{array}{l}C \\
C \\
C \\
C \\
C\end{array}$ & $\begin{array}{l}371 \\
371 \\
371 \\
371 \\
371\end{array}$ & $\begin{array}{l}\cdots \\
\cdots \\
\cdots \\
\cdots\end{array}$ & $\begin{array}{l}\cdots \\
\cdots \\
\cdots \\
\cdots\end{array}$ & $\begin{array}{l}3.1 \mathrm{ML} \\
2.7 \mathrm{ML} \\
2.7 \mathrm{ML} \\
2.7 \mathrm{ML} \\
3.1 \mathrm{ML}\end{array}$ & $\begin{array}{l}\text { GS } \\
\text { GS } \\
\text { GS } \\
\text { MMT } \\
\text { GS }\end{array}$ & $\begin{array}{l}\cdots \\
\cdots \\
\cdots \\
\cdots\end{array}$ & $\begin{array}{l}\cdots \\
\cdots \\
\cdots \\
\cdots \\
\cdots\end{array}$ & $\begin{array}{l}\cdots \\
\cdots \\
\cdots \\
\cdots \\
\cdots\end{array}$ & $\begin{array}{l}\ldots \\
\cdots \\
\cdots \\
\cdots\end{array}$ \\
\hline $\begin{array}{l}1985 \\
1985 \\
1985 \\
1985 \\
1985\end{array}$ & $\begin{array}{l}\text { AUG } \\
\text { AUG } \\
\text { AUG } \\
\text { AUG } \\
\text { SEP }\end{array}$ & $\begin{array}{l}28 \\
28 \\
30 \\
31 \\
04\end{array}$ & $\begin{array}{l}19 \\
20 \\
01 \\
00 \\
04\end{array}$ & $\begin{array}{l}10 \\
20 \\
14 \\
28 \\
07\end{array}$ & $\begin{array}{l}11.3 \\
37.8 \\
31.0 \\
49.2 \\
18.7\end{array}$ & $\begin{array}{l}44.483 \mathrm{~N} \\
44.425 \mathrm{~N} \\
44.507 \mathrm{~N} \\
44.427 \mathrm{~N} \\
44.209 \mathrm{~N} .\end{array}$ & $\begin{array}{l}114.215 W . \\
114.351 W . \\
114.324 W . \\
114.229 W . \\
114.140 W .\end{array}$ & $\begin{array}{l}005 \\
005 \\
009 \\
005 \\
005\end{array}$ & $\begin{array}{l}B \\
C \\
C \\
B \\
C\end{array}$ & $\begin{array}{l}371 \\
371 \\
371 \\
371 \\
371\end{array}$ & $\begin{array}{l}\cdots \\
\cdots \\
\cdots \\
\cdots\end{array}$ & $\begin{array}{l}\cdots \\
\cdots \\
\cdots \\
\cdots\end{array}$ & $\begin{array}{l}\text { 3. } 6 \mathrm{ML} \\
\text { 3. } 1 \mathrm{ML} \\
\text { 2.9ML } \\
\text { 3. } 3 \mathrm{ML} \\
\text { 3. } 9 \mathrm{ML}\end{array}$ & $\begin{array}{l}\text { GS } \\
\text { GS } \\
\text { MMT } \\
\text { GS } \\
\text { GS }\end{array}$ & $\begin{array}{l}\cdots \\
\cdots \\
\cdots \\
\cdots\end{array}$ & $\begin{array}{l}\cdots \\
\cdots \\
\cdots \\
\cdots \\
\cdots\end{array}$ & $\begin{array}{l}\cdots \\
\cdots \\
\cdots \\
\cdots \\
\cdots\end{array}$ & $\begin{array}{l}\cdots \\
\cdots \\
\cdots \\
\cdots\end{array}$ \\
\hline $\begin{array}{l}1985 \\
1985 \\
1985 \\
1985 \\
1985\end{array}$ & $\begin{array}{l}\text { SEP } \\
\text { SEP } \\
\text { OCT } \\
\text { OCT } \\
\text { OCT }\end{array}$ & $\begin{array}{l}23 \\
24 \\
02 \\
06 \\
06\end{array}$ & $\begin{array}{l}22 \\
08 \\
18 \\
03 \\
06\end{array}$ & $\begin{array}{l}07 \\
19 \\
48 \\
07 \\
07\end{array}$ & $\begin{array}{l}18.6 \\
29.3 \\
19.8 \\
55.0 \\
23.9\end{array}$ & $\begin{array}{l}44.263 \mathrm{~N} \\
44.434 \mathrm{~N} \\
44.345 \mathrm{~N} \\
44.341 \mathrm{~N} \\
44.364 \mathrm{~N}\end{array}$ & $\begin{array}{l}114.162 W . \\
114.276 W . \\
114.098 W . \\
114.263 W . \\
114.258 W .\end{array}$ & $\begin{array}{l}005 \\
005 \\
005 \\
005 \\
005\end{array}$ & $\begin{array}{l}C \\
C \\
C \\
C \\
C\end{array}$ & $\begin{array}{l}371 \\
371 \\
371 \\
371 \\
371\end{array}$ & $\begin{array}{l}\cdots \\
\cdots \\
\cdots \\
\cdots\end{array}$ & $\begin{array}{l}\cdots \\
\cdots \\
\cdots \\
\cdots\end{array}$ & $\begin{array}{l}2.8 \mathrm{ML} \\
2.8 \mathrm{ML} \\
3.6 \mathrm{ML} \\
3.0 \mathrm{ML} \\
2.4 \mathrm{ML}\end{array}$ & $\begin{array}{l}\text { GS } \\
\text { GS } \\
\text { MMT } \\
\text { MMT } \\
\text { GS }\end{array}$ & $\begin{array}{l}\cdots \\
\cdots \\
\cdots \\
\cdots\end{array}$ & $\begin{array}{l}\cdots \\
\cdots \\
\cdots \\
\cdots \\
\cdots\end{array}$ & $\begin{array}{l}\cdots \\
\cdots \\
\cdots \\
\cdots \\
\cdots\end{array}$ & $\begin{array}{l}\cdots \\
\ldots \\
\cdots \\
\cdots\end{array}$ \\
\hline $\begin{array}{l}1985 \\
1985 \\
1985 \\
1985 \\
1985\end{array}$ & $\begin{array}{l}\text { OCT } \\
\text { OCT } \\
\text { OCT } \\
\text { OCT } \\
\text { OCT }\end{array}$ & $\begin{array}{l}06 \\
09 \\
09 \\
19 \\
20\end{array}$ & $\begin{array}{l}06 \\
07 \\
09 \\
22 \\
02\end{array}$ & $\begin{array}{l}08 \\
18 \\
10 \\
42 \\
18\end{array}$ & $\begin{array}{l}25.8 \\
00.6 \\
03.0 \\
39.1 \\
51.6\end{array}$ & $\begin{array}{l}44.234 \mathrm{~N} \\
44.448 \mathrm{~N} \\
44.455 \mathrm{~N} \\
44.419 \mathrm{~N} \\
44.412 \mathrm{~N}\end{array}$ & $\begin{array}{l}114.088 W . \\
114.173 W . \\
114.154 W . \\
114.097 W . \\
114.256 W .\end{array}$ & $\begin{array}{l}021 \\
005 \\
005 \\
022 \\
020\end{array}$ & $\begin{array}{l}C \\
B \\
B \\
C \\
C\end{array}$ & $\begin{array}{l}371 \\
371 \\
371 \\
371 \\
371\end{array}$ & $\begin{array}{l}\cdots \\
\cdots \\
\cdots \\
\cdots\end{array}$ & $\begin{array}{l}\cdots \\
\cdots \\
\cdots \\
\cdots\end{array}$ & $\begin{array}{l}\text { 3. } 1 M L \\
\text { 3. } 3 M L \\
3.1 M L \\
2.7 M L \\
3.9 M L\end{array}$ & $\begin{array}{l}\text { MMT } \\
\text { GS } \\
\text { GS } \\
\text { MMT } \\
\text { MMT }\end{array}$ & $\begin{array}{l}\cdots \\
\cdots \\
\cdots \\
\cdots\end{array}$ & $\begin{array}{l}\cdots \\
\cdots \\
\cdots \\
\cdots \\
\cdots\end{array}$ & $\begin{array}{l}\cdots \\
\cdots \\
\cdots \\
\cdots \\
\cdots\end{array}$ & $\begin{array}{l}\cdots \\
\cdots \\
\cdots \\
\cdots\end{array}$ \\
\hline $\begin{array}{l}1985 \\
1985 \\
1985 \\
1985 \\
1985\end{array}$ & $\begin{array}{l}\text { OCT } \\
\text { OCT } \\
\text { OCT } \\
\text { OCT } \\
\text { OCT }\end{array}$ & $\begin{array}{l}20 \\
24 \\
26 \\
27 \\
29\end{array}$ & $\begin{array}{l}03 \\
05 \\
20 \\
09 \\
21\end{array}$ & $\begin{array}{l}15 \\
50 \\
57 \\
01 \\
30\end{array}$ & $\begin{array}{l}56.2 \\
13.2 \\
42.3 \\
41.2 \\
34.6\end{array}$ & $\begin{array}{l}44.548 \mathrm{~N} \\
44.556 \mathrm{~N} . \\
44.666 \mathrm{~N} \\
44.688 \mathrm{~N} \\
44.392 \mathrm{~N}\end{array}$ & $\begin{array}{l}114.283 W . \\
114.080 W . \\
114.687 W . \\
114.692 W . \\
114.075 W .\end{array}$ & $\begin{array}{l}005 \\
024 \\
022 \\
020 \\
005\end{array}$ & $\begin{array}{l}C \\
C \\
C \\
C \\
B\end{array}$ & $\begin{array}{l}371 \\
371 \\
371 \\
371 \\
371\end{array}$ & $\begin{array}{l}\cdots \\
\cdots \\
\cdots \\
\cdots\end{array}$ & $\begin{array}{l}\cdots \\
\cdots \\
\cdots \\
\cdots\end{array}$ & $\begin{array}{l}3.9 \mathrm{ML} \\
2.9 \mathrm{ML} \\
2.7 \mathrm{ML} \\
2.5 \mathrm{ML} \\
4.1 \mathrm{ML}\end{array}$ & $\begin{array}{l}\text { GS } \\
\text { MMT } \\
\text { MMT } \\
\text { MMT } \\
\text { GS }\end{array}$ & $\begin{array}{l}\cdots \\
\cdots \\
\cdots \\
\cdots\end{array}$ & $\begin{array}{l}\ldots \\
\ldots \\
\ldots \\
\ldots \\
\ldots\end{array}$ & $\begin{array}{l}\cdots \\
\cdots \\
\cdots \\
\cdots\end{array}$ & $\begin{array}{c}\ldots \\
\ldots \\
371\end{array}$ \\
\hline $\begin{array}{l}1985 \\
1985 \\
1985 \\
1985 \\
1985\end{array}$ & $\begin{array}{l}\text { OCT } \\
\text { OCT } \\
\text { OCT } \\
\text { NOV } \\
\text { NOV }\end{array}$ & $\begin{array}{l}29 \\
29 \\
31 \\
14 \\
18\end{array}$ & $\begin{array}{l}21 \\
23 \\
11 \\
09 \\
17\end{array}$ & $\begin{array}{l}37 \\
57 \\
31 \\
07 \\
30\end{array}$ & $\begin{array}{l}39.1 \\
38.4 \\
50.9 \\
58.2 \\
47.5\end{array}$ & $\begin{array}{l}44.408 \mathrm{~N} . \\
44.378 \mathrm{~N} . \\
47.514 \mathrm{~N} . \\
44.201 \mathrm{~N} . \\
44.314 \mathrm{~N} .\end{array}$ & $\begin{array}{l}114.030 W . \\
114.155 W . \\
115.751 W . \\
113.952 W . \\
114.094 W .\end{array}$ & $\begin{array}{l}018 \\
005 \\
005 \\
020 \\
018\end{array}$ & $\begin{array}{l}\mathrm{C} \\
\mathrm{B} \\
\mathrm{B} \\
\mathrm{C} \\
\mathrm{C}\end{array}$ & $\begin{array}{l}371 \\
371 \\
371 \\
371 \\
371\end{array}$ & $\begin{array}{l}\cdots \\
\cdots \\
\cdots \\
\cdots\end{array}$ & $\begin{array}{l}\cdots \\
\cdots \\
\cdots \\
\cdots\end{array}$ & $\begin{array}{l}2.8 \mathrm{ML} \\
3.2 \mathrm{ML} \\
2.5 \mathrm{ML} \\
2.6 \mathrm{ML} \\
3.2 \mathrm{ML}\end{array}$ & $\begin{array}{l}\text { MMT } \\
\text { GS } \\
\text { MMT } \\
\text { MMT } \\
\text { MMT }\end{array}$ & $\begin{array}{l}\cdots \\
\cdots \\
\cdots \\
\cdots\end{array}$ & $\begin{array}{l}\cdots \\
\cdots \\
\cdots \\
\cdots \\
\cdots\end{array}$ & $\begin{array}{l}\cdots \\
\cdots \\
\cdots \\
\cdots \\
\cdots\end{array}$ & $\begin{array}{l}\cdots \\
\cdots \\
\cdots \\
\cdots\end{array}$ \\
\hline $\begin{array}{l}1985 \\
1985 \\
1985 \\
1985 \\
1985\end{array}$ & $\begin{array}{l}\text { NOV } \\
\text { NOV } \\
\text { DEC } \\
\text { DEC } \\
\text { DEC }\end{array}$ & $\begin{array}{l}19 \\
22 \\
01 \\
04 \\
05\end{array}$ & $\begin{array}{l}09 \\
11 \\
20 \\
23 \\
16\end{array}$ & $\begin{array}{l}45 \\
43 \\
28 \\
40 \\
12\end{array}$ & $\begin{array}{l}09.4 \\
46.6 \\
04.7 \\
51.9 \\
46.4\end{array}$ & $\begin{array}{l}44.184 \mathrm{~N} \\
44.465 \mathrm{~N} \\
44.074 \mathrm{~N} \\
47.494 \mathrm{~N} \\
42.389 \mathrm{~N}\end{array}$ & $\begin{array}{l}116.168 W . \\
114.080 W . \\
114.051 W . \\
115.776 W . \\
111.572 W .\end{array}$ & $\begin{array}{l}014 \\
021 \\
005 \\
006 \\
007\end{array}$ & $\begin{array}{l}C \\
C \\
C \\
C \\
A\end{array}$ & $\begin{array}{l}371 \\
371 \\
371 \\
371 \\
371\end{array}$ & $\begin{array}{l}\cdots \\
\cdots \\
\cdots \\
\cdots\end{array}$ & $\begin{array}{l}\cdots \\
\cdots \\
\cdots \\
\cdots\end{array}$ & $\begin{array}{l}2.7 \mathrm{ML} \\
3.2 \mathrm{ML} \\
3.4 \mathrm{ML} \\
2.9 \mathrm{ML} \\
2.9 \mathrm{MD}\end{array}$ & $\begin{array}{l}\text { MMT } \\
\text { MMT } \\
\text { GS } \\
\text { MMT } \\
\text { UU }\end{array}$ & $\begin{array}{l}\cdots \\
\cdots \\
\cdots \\
\cdots\end{array}$ & $\begin{array}{l}\ldots \\
\ldots \\
\cdots \\
\ldots \\
\cdots\end{array}$ & $\begin{array}{l}\cdots \\
\cdots \\
\cdots \\
\cdots \\
\cdots\end{array}$ & $\begin{array}{l}\cdots \\
\cdots \\
\cdots \\
\cdots\end{array}$ \\
\hline $\begin{array}{l}1985 \\
1985 \\
1985 \\
1985 \\
1985\end{array}$ & $\begin{array}{l}\text { DEC } \\
\text { DEC } \\
\text { DEC } \\
\text { DEC } \\
\text { DEC }\end{array}$ & $\begin{array}{l}12 \\
12 \\
14 \\
22 \\
22\end{array}$ & $\begin{array}{l}10 \\
11 \\
14 \\
08 \\
12\end{array}$ & $\begin{array}{l}26 \\
27 \\
02 \\
01 \\
21\end{array}$ & $\begin{array}{l}40.5 \\
37.4 \\
23.8 \\
42.2 \\
30.3\end{array}$ & $\begin{array}{l}44.546 \mathrm{~N} \\
44.525 \mathrm{~N} \\
44.326 \mathrm{~N} \\
44.404 \mathrm{~N} \\
44.565 \mathrm{~N}\end{array}$ & $\begin{array}{l}113.053 W . \\
114.095 W . \\
114.204 W . \\
114.037 W . \\
114.018 W .\end{array}$ & $\begin{array}{l}005 \\
009 \\
005 \\
019 \\
001\end{array}$ & $\begin{array}{l}B \\
C \\
C \\
C \\
C\end{array}$ & $\begin{array}{l}371 \\
371 \\
371 \\
371 \\
371\end{array}$ & $\begin{array}{l}\cdots \\
\cdots \\
\cdots \\
\cdots\end{array}$ & $\begin{array}{l}\cdots \\
\cdots \\
\cdots \\
\cdots\end{array}$ & $\begin{array}{l}\text { 3. } 9 \mathrm{ML} \\
2.7 \mathrm{ML} \\
2.7 \mathrm{ML} \\
\text { 3. } \mathrm{OML} \\
2.9 \mathrm{ML}\end{array}$ & $\begin{array}{l}\text { GS } \\
\text { MMT } \\
\text { GS } \\
\text { MMT } \\
\text { MMT }\end{array}$ & $\begin{array}{l}\cdots \\
\cdots \\
\cdots \\
\cdots\end{array}$ & $\begin{array}{l}\cdots \\
\cdots \\
\cdots \\
\cdots \\
\cdots\end{array}$ & $\begin{array}{l}\cdots \\
\cdots \\
\cdots \\
\cdots\end{array}$ & $\begin{array}{l}\cdots \\
\cdots \\
\cdots \\
\cdots\end{array}$ \\
\hline $\begin{array}{l}1986 \\
1986 \\
1986 \\
1986 \\
1986\end{array}$ & $\begin{array}{l}\text { JAN } \\
\text { JAN } \\
\text { JAN } \\
\text { JAN } \\
\text { JAN }\end{array}$ & $\begin{array}{l}05 \\
06 \\
10 \\
11 \\
15\end{array}$ & $\begin{array}{l}01 \\
04 \\
03 \\
19 \\
16\end{array}$ & $\begin{array}{l}54 \\
52 \\
12 \\
24 \\
05\end{array}$ & $\begin{array}{l}33.0 \\
04.6 \\
15.1 \\
55.5 \\
04.9\end{array}$ & $\begin{array}{l}44.305 \mathrm{~N} \\
44.092 \mathrm{~N} \\
44.623 \mathrm{~N} \\
44.654 \mathrm{~N} \\
44.813 \mathrm{~N}\end{array}$ & $\begin{array}{l}114.138 W . \\
113.941 W . \\
116.001 W . \\
113.902 W . \\
114.489 W .\end{array}$ & $\begin{array}{l}013 \\
024 \\
010 \\
001 \\
010\end{array}$ & $\begin{array}{l}c \\
C \\
C \\
C \\
C\end{array}$ & $\begin{array}{l}562 \\
562 \\
562 \\
562 \\
562\end{array}$ & $\begin{array}{l}\cdots \\
\cdots \\
\cdots \\
\cdots\end{array}$ & $\begin{array}{l}\cdots \\
\cdots \\
\cdots \\
\cdots\end{array}$ & $\begin{array}{l}2.8 \mathrm{ML} \\
2.5 \mathrm{ML} \\
2.9 \mathrm{MD} \\
2.7 \mathrm{ML} \\
2.8 \mathrm{ML}\end{array}$ & $\begin{array}{l}\text { MMT } \\
\text { MMT } \\
\text { MMT } \\
\text { MMT } \\
\text { MMT }\end{array}$ & $\begin{array}{l}\cdots \\
\cdots \\
\cdots \\
\cdots\end{array}$ & $\begin{array}{l}\ldots \\
\cdots \\
\cdots \\
\cdots \\
\cdots\end{array}$ & $\begin{array}{l}\ldots \\
\cdots \\
\cdots \\
\cdots \\
\cdots\end{array}$ & $\begin{array}{l}\ldots \\
\ldots \\
\cdots \\
\cdots\end{array}$ \\
\hline $\begin{array}{l}1986 \\
1986 \\
1986 \\
1986 \\
1986\end{array}$ & $\begin{array}{l}\text { JAN } \\
\text { JAN } \\
\text { JAN } \\
\text { JAN } \\
\text { JAN }\end{array}$ & $\begin{array}{l}16 \\
16 \\
18 \\
28 \\
28\end{array}$ & $\begin{array}{l}02 \\
11 \\
14 \\
05 \\
07\end{array}$ & $\begin{array}{l}01 \\
27 \\
39 \\
45 \\
15\end{array}$ & $\begin{array}{l}58.4 \\
54.0 \\
50.3 \\
01.5 \\
32.8\end{array}$ & $\begin{array}{l}44.447 \mathrm{~N} \\
44.476 \mathrm{~N} \\
44.412 \mathrm{~N} \\
44.153 \mathrm{~N} \\
44.184 \mathrm{~N}\end{array}$ & $\begin{array}{l}114.232 W . \\
114.108 W . \\
113.802 W . \\
113.946 W . \\
113.955 W .\end{array}$ & $\begin{array}{l}005 \\
013 \\
028 \\
005 \\
005\end{array}$ & $\begin{array}{l}C \\
C \\
C \\
B \\
B\end{array}$ & $\begin{array}{l}562 \\
562 \\
562 \\
562 \\
562\end{array}$ & $\begin{array}{l}\cdots \\
\cdots \\
\cdots \\
\cdots\end{array}$ & $\begin{array}{l}\cdots \\
\cdots \\
\cdots \\
\cdots\end{array}$ & $\begin{array}{l}\text { 3. } 1 \mathrm{ML} \\
\text { 2.8ML } \\
\text { 2. } 7 \mathrm{ML} \\
\text { 4. } 0 \mathrm{ML} \\
\text { 3. } 7 \mathrm{ML}\end{array}$ & $\begin{array}{l}\text { GS } \\
\text { MMT } \\
\text { MMT } \\
\text { GS } \\
\text { GS }\end{array}$ & $\begin{array}{l}\cdots \\
\cdots \\
\cdots \\
\cdots\end{array}$ & $\begin{array}{l}\cdots \\
\cdots \\
\cdots \\
\cdots \\
\cdots\end{array}$ & $\begin{array}{l}\cdots \\
\cdots \\
\text { IV } \\
\cdots\end{array}$ & $\begin{array}{r}\ldots \\
\ldots \\
562 \\
\ldots\end{array}$ \\
\hline 1986 & JAN & 28 & 07 & 26 & 57.5 & $44.111 \mathrm{~N}$ & $113.901 w$. & 024 & C & 562 & $\cdots$ & . & 3. 3ML & GS & $\cdots$ & $\cdots$ & $\cdots$ & $\cdots$ \\
\hline
\end{tabular}




\begin{tabular}{|c|c|c|c|c|c|c|c|c|c|c|c|c|c|c|c|c|c|c|}
\hline $\begin{array}{l}1986 \\
1986 \\
1986 \\
1986\end{array}$ & $\begin{array}{l}\text { JAN } \\
\text { FEB } \\
\text { FEB } \\
\text { FEB }\end{array}$ & $\begin{array}{l}30 \\
05 \\
09 \\
10\end{array}$ & $\begin{array}{l}11 \\
15 \\
07 \\
00\end{array}$ & $\begin{array}{l}47 \\
25 \\
03 \\
17\end{array}$ & $\begin{array}{l}55.3 \\
28.1 \\
51.3 \\
43.7\end{array}$ & $\begin{array}{l}44.780 N \\
44.234 N \\
44.363 N \\
44.644 N\end{array}$ & $\begin{array}{l}111.491 W . \\
114.006 W . \\
113.930 W . \\
114.712 W .\end{array}$ & $\begin{array}{l}020 \\
018 \\
029 \\
021\end{array}$ & $\begin{array}{l}c \\
c \\
c \\
c\end{array}$ & $\begin{array}{l}562 \\
562 \\
562 \\
562\end{array}$ & $\begin{array}{l}\cdots \\
\cdots \\
\cdots \\
\cdots\end{array}$ & $\begin{array}{l}\cdots \\
\cdots \\
\cdots\end{array}$ & $\begin{array}{l}2.5 \mathrm{ML} \\
3.3 \mathrm{ML} \\
3.1 \mathrm{ML} \\
2.6 \mathrm{ML}\end{array}$ & $\begin{array}{l}\text { MMT } \\
\text { MMT } \\
\text { MMT } \\
\text { MMT }\end{array}$ & $\begin{array}{l}\cdots \\
\cdots \\
\cdots\end{array}$ & $\begin{array}{l}\cdots \\
\cdots \\
\cdots \\
\cdots\end{array}$ & $\begin{array}{l}\cdots \\
\cdots \\
\cdots \\
\cdots\end{array}$ & $\begin{array}{l}\cdots \\
\cdots \\
\cdots\end{array}$ \\
\hline $\begin{array}{l}1986 \\
1986 \\
1986 \\
1986 \\
1986\end{array}$ & $\begin{array}{l}\text { FEB } \\
\text { FEB } \\
\text { FEB } \\
\text { FEB } \\
\text { FEB }\end{array}$ & $\begin{array}{l}16 \\
17 \\
22 \\
24 \\
26\end{array}$ & $\begin{array}{l}12 \\
08 \\
18 \\
03 \\
15\end{array}$ & $\begin{array}{l}54 \\
53 \\
07 \\
13 \\
05\end{array}$ & $\begin{array}{l}44.0 \\
38.6 \\
18.7 \\
33.0 \\
49.3\end{array}$ & $\begin{array}{l}44.627 \mathrm{~N} \\
42.596 \mathrm{~N} \\
44.578 \mathrm{~N} \\
43.081 \mathrm{~N} \\
44.627 \mathrm{~N}\end{array}$ & $\begin{array}{l}114.088 W . \\
111.301 W . \\
114.232 W . \\
111.224 W . \\
114.192 W .\end{array}$ & $\begin{array}{l}013 \\
005 \\
010 \\
005 \\
005\end{array}$ & $\begin{array}{l}\mathrm{C} \\
\mathrm{B} \\
\mathrm{C} \\
\mathrm{C} \\
\mathrm{B}\end{array}$ & $\begin{array}{l}562 \\
562 \\
562 \\
562 \\
562\end{array}$ & $\begin{array}{l}\cdots \\
\cdots \\
\cdots \\
\cdots\end{array}$ & $\begin{array}{l}\cdots \\
\cdots \\
\cdots \\
\cdots\end{array}$ & $\begin{array}{l}2.8 \mathrm{ML} \\
3.0 \mathrm{ML} \\
2.7 \mathrm{ML} \\
2.8 \mathrm{ML} \\
3.7 \mathrm{ML}\end{array}$ & $\begin{array}{l}\text { MMT } \\
\text { GS } \\
\text { MMT } \\
\text { GS } \\
\text { GS }\end{array}$ & $\begin{array}{l}\cdots \\
\cdots \\
\cdots \\
\cdots\end{array}$ & $\begin{array}{l}\cdots \\
\cdots \\
\cdots \\
\cdots \\
\cdots\end{array}$ & $\begin{array}{l}\because \ddot{I} \text { I } \\
\text { III } \\
\ldots\end{array}$ & $\begin{array}{r}5 \dot{62} \\
5 \dot{62} \\
\ldots\end{array}$ \\
\hline $\begin{array}{l}1986 \\
1986 \\
1986 \\
1986 \\
1986\end{array}$ & $\begin{array}{l}\text { FEB } \\
\text { MAR } \\
\text { MAR } \\
\text { MAR } \\
\text { MAR }\end{array}$ & $\begin{array}{l}28 \\
08 \\
11 \\
12 \\
23\end{array}$ & $\begin{array}{l}22 \\
20 \\
22 \\
16 \\
14\end{array}$ & $\begin{array}{l}09 \\
58 \\
57 \\
32 \\
26\end{array}$ & $\begin{array}{l}10.1 \\
35.2 \\
04.9 \\
56.0 \\
11.8\end{array}$ & $\begin{array}{l}44.328 \mathrm{~N} \\
44.397 \mathrm{~N} \\
44.485 \mathrm{~N} \\
47.470 \mathrm{~N} \\
44.407 \mathrm{~N}\end{array}$ & $\begin{array}{l}114.080 w . \\
113.999 w . \\
114.146 w . \\
115.800 w . \\
114.213 w .\end{array}$ & $\begin{array}{l}022 \\
004 \\
019 \\
001 \\
005\end{array}$ & $\begin{array}{l}\text { C } \\
\text { C } \\
\text { B } \\
\text { A } \\
\text { B }\end{array}$ & $\begin{array}{l}562 \\
562 \\
562 \\
562 \\
562\end{array}$ & $\begin{array}{l}\cdots \\
\cdots \\
\cdots \\
\cdots\end{array}$ & $\begin{array}{l}\cdots \\
\cdots \\
\cdots \\
\cdots\end{array}$ & $\begin{array}{l}2.6 \mathrm{ML} \\
2.8 \mathrm{ML} \\
3.2 \mathrm{ML} \\
2.6 \mathrm{ML} \\
3.1 \mathrm{ML}\end{array}$ & $\begin{array}{l}\text { MMT } \\
\text { MMT } \\
\text { MMT } \\
\text { MMT } \\
\text { GS }\end{array}$ & $\begin{array}{l}\cdots \\
\cdots \\
\cdots \\
\cdots\end{array}$ & $\begin{array}{l}\ldots \\
\cdots \\
\cdots \\
\cdots \\
\cdots\end{array}$ & $\begin{array}{l}\cdots \\
\cdots \\
\cdots \\
\cdots\end{array}$ & $\begin{array}{c}\ldots \\
\ldots \\
562 \\
\ldots\end{array}$ \\
\hline $\begin{array}{l}1986 \\
1986 \\
1986 \\
1986 \\
1986\end{array}$ & $\begin{array}{l}\text { MAR } \\
\text { APR } \\
\text { APR } \\
\text { APR } \\
\text { APR }\end{array}$ & $\begin{array}{l}31 \\
07 \\
11 \\
11 \\
13\end{array}$ & $\begin{array}{l}18 \\
14 \\
09 \\
10 \\
05\end{array}$ & $\begin{array}{l}13 \\
07 \\
31 \\
13 \\
02\end{array}$ & $\begin{array}{l}51.3 \\
25.8 \\
29.1 \\
58.7 \\
50.1\end{array}$ & $\begin{array}{l}44.251 \mathrm{~N} . \\
44.337 \mathrm{~N} \\
44.240 \mathrm{~N} \\
44.572 \mathrm{~N} \\
44.283 \mathrm{~N}\end{array}$ & $\begin{array}{l}114.668 W . \\
114.177 W . \\
114.004 W . \\
114.294 W . \\
114.153 W .\end{array}$ & $\begin{array}{l}034 \\
005 \\
022 \\
018 \\
005\end{array}$ & $\begin{array}{l}C \\
B \\
C \\
C \\
C\end{array}$ & $\begin{array}{l}562 \\
562 \\
562 \\
562 \\
562\end{array}$ & $\begin{array}{l}\cdots \\
\cdots \\
\cdots \\
\cdots\end{array}$ & $\begin{array}{l}\cdots \\
\cdots \\
\cdots \\
\cdots\end{array}$ & $\begin{array}{l}\text { 2. } 7 \mathrm{ML} \\
\text { 4. } 1 \mathrm{ML} \\
2.7 \mathrm{ML} \\
2.8 \mathrm{ML} \\
\text { 3. } 8 \mathrm{ML}\end{array}$ & $\begin{array}{l}\text { MMT } \\
\text { GS } \\
\text { MMT } \\
\text { MMT } \\
\text { GS }\end{array}$ & $\begin{array}{l}\cdots \\
\cdots \\
\cdots \\
\cdots\end{array}$ & $\begin{array}{l}\ldots \\
\cdots \\
\cdots \\
\cdots \\
\cdots\end{array}$ & $\begin{array}{l}\because \text { II I } \\
\ldots \\
\ldots \\
\cdots\end{array}$ & $\begin{array}{r}5 \dot{62} \\
\ldots \\
\cdots \\
\cdots\end{array}$ \\
\hline $\begin{array}{l}1986 \\
1986 \\
1986 \\
1986 \\
1986\end{array}$ & $\begin{array}{l}\text { APR } \\
\text { APR } \\
\text { APR } \\
\text { APR } \\
\text { APR }\end{array}$ & $\begin{array}{l}14 \\
15 \\
16 \\
16 \\
17\end{array}$ & $\begin{array}{l}10 \\
06 \\
06 \\
17 \\
03\end{array}$ & $\begin{array}{l}02 \\
05 \\
25 \\
54 \\
10\end{array}$ & $\begin{array}{l}55.5 \\
50.4 \\
27.6 \\
47.2 \\
38.9\end{array}$ & $\begin{array}{l}44.619 \mathrm{~N} \\
44.310 \mathrm{~N} \\
44.271 \mathrm{~N} \\
44.613 \mathrm{~N} \\
44.323 \mathrm{~N}\end{array}$ & $\begin{array}{l}113.992 W . \\
114.172 W . \\
114.099 w . \\
114.423 w . \\
114.119 w .\end{array}$ & $\begin{array}{l}023 \\
017 \\
005 \\
017 \\
005\end{array}$ & $\begin{array}{l}B \\
C \\
B \\
C \\
C\end{array}$ & $\begin{array}{l}562 \\
562 \\
562 \\
562 \\
562\end{array}$ & $\begin{array}{l}\cdots \\
\cdots \\
\cdots \\
\cdots\end{array}$ & $\begin{array}{l}\cdots \\
\cdots \\
\cdots \\
\cdots\end{array}$ & $\begin{array}{l}\text { 3. } 9 \mathrm{ML} \\
3.7 \mathrm{ML} \\
3.5 \mathrm{ML} \\
2.5 \mathrm{ML} \\
3.0 \mathrm{ML}\end{array}$ & $\begin{array}{l}\text { MMT } \\
\text { MMT } \\
\text { GS } \\
\text { MMT } \\
\text { GS }\end{array}$ & $\begin{array}{l}\cdots \\
\cdots \\
\cdots \\
\cdots\end{array}$ & $\begin{array}{l}\cdots \\
\cdots \\
\cdots \\
\cdots \\
\cdots\end{array}$ & $\begin{array}{l}\cdots \\
\cdots \\
\cdots \\
\cdots \\
\cdots\end{array}$ & $\begin{array}{l}\cdots \\
\cdots \\
\cdots \\
\cdots\end{array}$ \\
\hline $\begin{array}{l}1986 \\
1986 \\
1986 \\
1986 \\
1986\end{array}$ & $\begin{array}{l}\text { APR } \\
\text { APR } \\
\text { APR } \\
\text { APR } \\
\text { MAY }\end{array}$ & $\begin{array}{l}20 \\
20 \\
28 \\
28 \\
15\end{array}$ & $\begin{array}{l}02 \\
17 \\
12 \\
12 \\
15\end{array}$ & $\begin{array}{l}31 \\
12 \\
00 \\
51 \\
06\end{array}$ & $\begin{array}{l}55.3 \\
25.4 \\
23.8 \\
08.3 \\
26.3\end{array}$ & $\begin{array}{l}44.136 \mathrm{~N} \\
44.105 \mathrm{~N} \\
44.492 \mathrm{~N} \\
44.573 \mathrm{~N} \\
44.687 \mathrm{~N}\end{array}$ & $\begin{array}{l}114.925 w . \\
113.823 w . \\
114.843 w . \\
114.879 w . \\
114.444 W .\end{array}$ & $\begin{array}{l}005 \\
021 \\
008 \\
005 \\
005\end{array}$ & $\begin{array}{l}C \\
C \\
C \\
C \\
C\end{array}$ & $\begin{array}{l}562 \\
562 \\
562 \\
562 \\
562\end{array}$ & $\begin{array}{l}\cdots \\
\cdots \\
\cdots \\
\cdots\end{array}$ & $\begin{array}{l}\cdots \\
\cdots \\
\cdots \\
\cdots\end{array}$ & $\begin{array}{l}2.8 \mathrm{ML} \\
2.6 \mathrm{ML} \\
2.8 \mathrm{ML} \\
2.5 \mathrm{ML} \\
2.6 \mathrm{ML}\end{array}$ & $\begin{array}{l}\text { GS } \\
\text { MMT } \\
\text { MMT } \\
\text { GS } \\
\text { MMT }\end{array}$ & $\begin{array}{l}\cdots \\
\cdots \\
\cdots \\
\cdots\end{array}$ & $\begin{array}{l}\cdots \\
\cdots \\
\cdots \\
\cdots \\
\cdots\end{array}$ & $\begin{array}{l}\cdots \\
\cdots \\
\cdots \\
\cdots \\
\cdots\end{array}$ & $\begin{array}{l}\cdots \\
\cdots \\
\cdots \\
\cdots\end{array}$ \\
\hline $\begin{array}{l}1986 \\
1986 \\
1986 \\
1986 \\
1986\end{array}$ & $\begin{array}{l}\text { MAY } \\
\text { MAY } \\
\text { MAY } \\
\text { JUN } \\
\text { JUN }\end{array}$ & $\begin{array}{l}17 \\
19 \\
21 \\
02 \\
21\end{array}$ & $\begin{array}{l}04 \\
02 \\
03 \\
22 \\
20\end{array}$ & $\begin{array}{l}07 \\
17 \\
04 \\
56 \\
30\end{array}$ & $\begin{array}{l}48.7 \\
10.0 \\
57.6 \\
38.8 \\
53.5\end{array}$ & $\begin{array}{l}44.187 N \\
44.583 N \\
44.658 N \\
44.259 N \\
42.793 N\end{array}$ & $\begin{array}{l}113.947 W . \\
115.180 W . \\
113.984 W . \\
114.061 W . \\
111.153 w .\end{array}$ & $\begin{array}{l}012 \\
032 \\
006 \\
018 \\
005\end{array}$ & $\begin{array}{l}C \\
C \\
C \\
C \\
B\end{array}$ & $\begin{array}{l}562 \\
562 \\
562 \\
562 \\
562\end{array}$ & $\begin{array}{l}\cdots \\
\cdots \\
\cdots \\
\cdots\end{array}$ & $\begin{array}{l}\ldots \\
\ldots \\
\cdots \\
\cdots\end{array}$ & $\begin{array}{l}\text { 3. } 4 \mathrm{ML} \\
2.9 \mathrm{ML} \\
2.6 \mathrm{ML} \\
2.7 \mathrm{ML} \\
3.5 \mathrm{ML}\end{array}$ & $\begin{array}{l}\text { MMT } \\
\text { MMT } \\
\text { MMT } \\
\text { MMT } \\
\text { GS }\end{array}$ & $\begin{array}{l}\cdots \\
\cdots \\
\cdots \\
\cdots\end{array}$ & $\begin{array}{l}\cdots \\
\cdots \\
\cdots \\
\cdots \\
\cdots\end{array}$ & $\begin{array}{l}\cdots \\
\cdots \\
\cdots \\
\dot{I I I}\end{array}$ & $\begin{array}{c}\cdots \\
\cdots \\
\dot{562}\end{array}$ \\
\hline $\begin{array}{l}1986 \\
1986 \\
1986 \\
1986 \\
1986\end{array}$ & $\begin{array}{l}\text { JUL } \\
\text { JUL } \\
\text { JUL } \\
\text { JUL } \\
\text { JUL }\end{array}$ & $\begin{array}{l}07 \\
20 \\
20 \\
29 \\
30\end{array}$ & $\begin{array}{l}11 \\
02 \\
19 \\
19 \\
08\end{array}$ & $\begin{array}{l}53 \\
29 \\
05 \\
04 \\
19\end{array}$ & $\begin{array}{l}17.2 \\
20.3 \\
32.6 \\
18.9 \\
06.5\end{array}$ & $\begin{array}{l}43.248 \mathrm{~N} \\
44.415 \mathrm{~N} \\
44.455 \mathrm{~N} \\
44.247 \mathrm{~N} \\
42.452 \mathrm{~N}\end{array}$ & $\begin{array}{l}111.090 w . \\
116.002 W . \\
116.033 w . \\
114.136 w . \\
111.219 w .\end{array}$ & $\begin{array}{l}005 \\
005 \\
005 \\
005 \\
000\end{array}$ & $\begin{array}{l}B \\
B \\
B \\
B \\
A\end{array}$ & $\begin{array}{l}562 \\
562 \\
562 \\
562 \\
562\end{array}$ & $\begin{array}{l}\cdots \\
\cdots \\
\cdots \\
\cdots\end{array}$ & $\begin{array}{l}\cdots \\
\cdots \\
\cdots \\
\cdots\end{array}$ & $\begin{array}{l}\text { 3. } 3 \mathrm{ML} \\
3.2 \mathrm{ML} \\
3.6 \mathrm{ML} \\
3.5 \mathrm{ML} \\
3.5 \mathrm{ML}\end{array}$ & $\begin{array}{l}\text { GS } \\
\text { GS } \\
\text { GS } \\
\text { GS } \\
\text { GS }\end{array}$ & $\begin{array}{l}\cdots \\
\cdots \\
\cdots \\
\cdots\end{array}$ & $\begin{array}{l}\cdots \\
\cdots \\
\cdots \\
\cdots \\
\cdots\end{array}$ & $\begin{array}{l}\cdots \\
\cdots \\
\cdots \\
\cdots \\
\cdots\end{array}$ & $\begin{array}{l}\cdots \\
\cdots \\
\cdots \\
\cdots\end{array}$ \\
\hline $\begin{array}{l}1986 \\
1986 \\
1986 \\
1986 \\
1986\end{array}$ & $\begin{array}{l}\text { AUG } \\
\text { AUG } \\
\text { AUG } \\
\text { AUG } \\
\text { AUG }\end{array}$ & $\begin{array}{l}03 \\
06 \\
10 \\
17 \\
18\end{array}$ & $\begin{array}{l}04 \\
09 \\
10 \\
08 \\
20\end{array}$ & $\begin{array}{l}48 \\
37 \\
05 \\
59 \\
30\end{array}$ & $\begin{array}{l}16.4 \\
42.1 \\
58.1 \\
20.7 \\
21.7\end{array}$ & $\begin{array}{l}44.469 \mathrm{~N} \\
44.152 \mathrm{~N} \\
44.512 \mathrm{~N} \\
44.118 \mathrm{~N} \\
44.460 \mathrm{~N}\end{array}$ & $\begin{array}{l}114.150 W . \\
114.612 W . \\
114.261 W . \\
113.967 W . \\
114.190 W .\end{array}$ & $\begin{array}{l}014 \\
023 \\
020 \\
016 \\
018\end{array}$ & $\begin{array}{l}c \\
C \\
c \\
C \\
C\end{array}$ & $\begin{array}{l}562 \\
562 \\
562 \\
562 \\
562\end{array}$ & $\begin{array}{l}\cdots \\
\cdots \\
\cdots \\
\cdots\end{array}$ & $\begin{array}{l}\cdots \\
\ldots \\
\cdots \\
\cdots\end{array}$ & $\begin{array}{l}2.9 \mathrm{ML} \\
2.9 \mathrm{ML} \\
3 . \mathrm{ML} \\
2.6 \mathrm{ML} \\
2.8 \mathrm{ML}\end{array}$ & $\begin{array}{l}\text { MMT } \\
\text { MMT } \\
\text { MMT } \\
\text { MMT } \\
\text { MMT }\end{array}$ & $\begin{array}{l}\cdots \\
\cdots \\
\cdots \\
\cdots\end{array}$ & $\begin{array}{l}\cdots \\
\cdots \\
\cdots \\
\cdots \\
\cdots\end{array}$ & $\begin{array}{l}\cdots \\
\cdots \\
\cdots \\
\cdots \\
\cdots\end{array}$ & $\begin{array}{l}\cdots \\
\cdots \\
\cdots \\
\cdots\end{array}$ \\
\hline $\begin{array}{l}1986 \\
1986 \\
1986 \\
1986 \\
1986\end{array}$ & $\begin{array}{l}\text { AUG } \\
\text { AUG } \\
\text { AUG } \\
\text { SEP } \\
\text { SEP }\end{array}$ & $\begin{array}{l}28 \\
29 \\
29 \\
01 \\
03\end{array}$ & $\begin{array}{l}15 \\
08 \\
09 \\
02 \\
06\end{array}$ & $\begin{array}{l}12 \\
26 \\
37 \\
03 \\
11\end{array}$ & $\begin{array}{l}58.9 \\
24.1 \\
34.6 \\
24.6 \\
11.3\end{array}$ & $\begin{array}{l}44.215 \mathrm{~N} \\
42.106 \mathrm{~N} \\
42.105 \mathrm{~N} \\
44.460 \mathrm{~N} \\
44.010 \mathrm{~N}\end{array}$ & $\begin{array}{l}114.260 w \text {. } \\
111.655 w . \\
111.652 W . \\
114.280 w . \\
114.792 W .\end{array}$ & $\begin{array}{l}019 \\
001 \\
001 \\
021 \\
005\end{array}$ & $\begin{array}{l}C \\
A \\
B \\
C \\
C\end{array}$ & $\begin{array}{l}562 \\
562 \\
562 \\
562 \\
562\end{array}$ & $\begin{array}{l}\cdots \\
\cdots \\
\cdots \\
\cdots\end{array}$ & $\begin{array}{l}\cdots \\
\cdots \\
\cdots \\
\cdots\end{array}$ & $\begin{array}{l}\text { 2. } 8 \mathrm{ML} \\
\text { 3. } 2 \mathrm{ML} \\
\text { 2. } 4 \mathrm{ML} \\
\text { 2. } 7 \mathrm{ML} \\
\text { 3. } 2 \mathrm{ML}\end{array}$ & $\begin{array}{l}\text { MMT } \\
\text { UU } \\
\text { UU } \\
\text { MMT } \\
\text { GS }\end{array}$ & $\begin{array}{l}\cdots \\
\cdots \\
\cdots \\
\cdots\end{array}$ & $\begin{array}{l}\cdots \\
\cdots \\
\cdots \\
\cdots \\
\cdots\end{array}$ & $\begin{array}{l}\cdots \\
\text { III } \\
\cdots \\
\cdots\end{array}$ & $\begin{array}{c}\ldots \\
5 \dot{62} \\
\ldots \\
\ldots\end{array}$ \\
\hline $\begin{array}{l}1986 \\
1986 \\
1986 \\
1986 \\
1986\end{array}$ & $\begin{array}{l}\text { SEP } \\
\text { SEP } \\
\text { SEP } \\
\text { SEP } \\
\text { SEP }\end{array}$ & $\begin{array}{l}03 \\
04 \\
04 \\
04 \\
04\end{array}$ & $\begin{array}{l}18 \\
00 \\
04 \\
04 \\
12\end{array}$ & $\begin{array}{l}53 \\
14 \\
15 \\
38 \\
43\end{array}$ & $\begin{array}{l}49.1 \\
58.3 \\
55.8 \\
20.0 \\
47.6\end{array}$ & $\begin{array}{l}44.039 \mathrm{~N} \\
43.970 \mathrm{~N} \\
43.993 \mathrm{~N} \\
44.066 \mathrm{~N} \\
44.036 \mathrm{~N}\end{array}$ & $\begin{array}{l}114.764 W . \\
114.658 W . \\
114.803 W . \\
114.745 W . \\
114.719 W .\end{array}$ & $\begin{array}{l}005 \\
024 \\
005 \\
005 \\
005\end{array}$ & $\begin{array}{l}B \\
B \\
C \\
B \\
B\end{array}$ & $\begin{array}{l}562 \\
562 \\
562 \\
562 \\
562\end{array}$ & $\begin{array}{l}\cdots \\
\cdots \\
\cdots \\
\cdots\end{array}$ & $\begin{array}{l}\cdots \\
\cdots \\
\cdots \\
\cdots\end{array}$ & $\begin{array}{l}\text { 3. } 9 \mathrm{ML} \\
2.9 \mathrm{ML} \\
3.4 \mathrm{ML} \\
4.9 \mathrm{ML} \\
\text { 3. } 3 \mathrm{ML}\end{array}$ & $\begin{array}{l}\text { GS } \\
\text { MMT } \\
\text { GS } \\
\text { GS } \\
\text { GS }\end{array}$ & $\begin{array}{l}\cdots \\
\cdots \\
\cdots \\
\cdots\end{array}$ & $\begin{array}{l}\cdots \\
\cdots \\
\cdots \\
\cdots \\
\cdots\end{array}$ & $\begin{array}{l}\text { II I } \\
\cdots \\
\cdots \\
\cdots \\
\cdots\end{array}$ & $\begin{array}{c}562 \\
\ldots \\
\ldots \\
\ldots \\
\ldots\end{array}$ \\
\hline $\begin{array}{l}1986 \\
1986 \\
1986 \\
1986 \\
1986\end{array}$ & $\begin{array}{l}\text { SEP } \\
\text { SEP } \\
\text { SEP } \\
\text { SEP } \\
\text { SEP }\end{array}$ & $\begin{array}{l}05 \\
05 \\
05 \\
05 \\
05\end{array}$ & $\begin{array}{l}16 \\
19 \\
19 \\
19 \\
19\end{array}$ & $\begin{array}{l}05 \\
15 \\
20 \\
23 \\
40\end{array}$ & $\begin{array}{l}05.5 \\
05.2 \\
51.0 \\
51.3 \\
05.3\end{array}$ & $\begin{array}{l}43.989 \mathrm{~N} \\
43.984 \mathrm{~N} \\
44.008 \mathrm{~N} \\
44.017 \mathrm{~N} \\
43.986 \mathrm{~N}\end{array}$ & $\begin{array}{l}114.656 w . \\
114.645 W . \\
114.752 W . \\
114.693 w . \\
114.673 w .\end{array}$ & $\begin{array}{l}019 \\
021 \\
005 \\
019 \\
019\end{array}$ & $\begin{array}{l}B \\
B \\
B \\
B \\
B\end{array}$ & $\begin{array}{l}562 \\
562 \\
562 \\
562 \\
562\end{array}$ & $\begin{array}{l}\cdots \\
\cdots \\
\cdots \\
\cdots\end{array}$ & $\begin{array}{l}\cdots \\
\cdots \\
\cdots \\
\cdots\end{array}$ & $\begin{array}{l}\text { 3. } 7 \mathrm{ML} \\
\text { 3. } 2 \mathrm{ML} \\
\text { 4. } \mathrm{OML} \\
\text { 3. } 4 \mathrm{ML} \\
\text { 3. } 3 \mathrm{ML}\end{array}$ & $\begin{array}{l}\text { MMT } \\
\text { MMT } \\
\text { GS } \\
\text { GS } \\
\text { MMT }\end{array}$ & $\begin{array}{l}\cdots \\
\cdots \\
\cdots \\
\cdots\end{array}$ & $\begin{array}{l}\cdots \\
\cdots \\
\cdots \\
\cdots \\
\cdots\end{array}$ & $\begin{array}{l}\cdots \\
\cdots \\
\cdots \\
\cdots \\
\cdots\end{array}$ & $\begin{array}{l}\cdots \\
\cdots \\
\cdots \\
\cdots\end{array}$ \\
\hline
\end{tabular}




\begin{tabular}{|c|c|c|c|c|c|c|c|c|c|c|c|c|c|c|c|c|c|c|}
\hline $\begin{array}{l}1986 \\
1986 \\
1986 \\
1986 \\
1986\end{array}$ & $\begin{array}{l}\text { SEP } \\
\text { SEP } \\
\text { SEP } \\
\text { SEP } \\
\text { SEP }\end{array}$ & $\begin{array}{l}06 \\
06 \\
06 \\
07 \\
07\end{array}$ & $\begin{array}{l}02 \\
17 \\
21 \\
10 \\
11\end{array}$ & $\begin{array}{l}31 \\
55 \\
40 \\
35 \\
07\end{array}$ & $\begin{array}{l}34.9 \\
22.7 \\
27.5 \\
53.7 \\
47.8\end{array}$ & $\begin{array}{l}43.974 \mathrm{~N} \\
44.012 \mathrm{~N} \\
43.984 \mathrm{~N} \\
43.960 \mathrm{~N} \\
43.984 \mathrm{~N}\end{array}$ & $\begin{array}{l}114.649 w . \\
114.659 w . \\
114.656 w . \\
114.647 w . \\
114.657 w .\end{array}$ & $\begin{array}{l}021 \\
019 \\
020 \\
022 \\
020\end{array}$ & $\begin{array}{l}\mathrm{B} \\
\mathrm{B} \\
\mathrm{B} \\
\mathrm{B} \\
\mathrm{B}\end{array}$ & $\begin{array}{l}562 \\
562 \\
562 \\
562 \\
562\end{array}$ & $\begin{array}{l}\cdots \\
\cdots \\
\cdots \\
\cdots\end{array}$ & $\begin{array}{l}\cdots \\
\cdots \\
\cdots \\
\cdots\end{array}$ & $\begin{array}{l}3.0 \mathrm{ML} \\
3.5 \mathrm{ML} \\
3.2 \mathrm{ML} \\
3.2 \mathrm{ML} \\
3.1 \mathrm{ML}\end{array}$ & $\begin{array}{l}\text { MMT } \\
\text { GS } \\
\text { MMT } \\
\text { MMT } \\
\text { MMT }\end{array}$ & $\begin{array}{l}\cdots \\
\cdots \\
\cdots \\
\cdots\end{array}$ & $\begin{array}{l}\cdots \\
\cdots \\
\cdots \\
\cdots \\
\cdots\end{array}$ & $\begin{array}{l}\ldots \\
\cdots \\
\cdots \\
\cdots \\
\cdots\end{array}$ & $\begin{array}{l}\ldots \\
\cdots \\
\cdots \\
\cdots\end{array}$ \\
\hline $\begin{array}{l}1986 \\
1986 \\
1986 \\
1986 \\
1986\end{array}$ & $\begin{array}{l}\text { SEP } \\
\text { SEP } \\
\text { SEP } \\
\text { SEP } \\
\text { SEP }\end{array}$ & $\begin{array}{l}11 \\
11 \\
11 \\
11 \\
11\end{array}$ & $\begin{array}{l}02 \\
02 \\
03 \\
03 \\
07\end{array}$ & $\begin{array}{l}07 \\
12 \\
49 \\
55 \\
09\end{array}$ & $\begin{array}{l}28.4 \\
34.2 \\
11.6 \\
12.7 \\
25.6\end{array}$ & $\begin{array}{l}44.053 \mathrm{~N} \\
44.057 \mathrm{~N} . \\
43.960 \mathrm{~N} . \\
44.067 \mathrm{~N} \\
43.990 \mathrm{~N} .\end{array}$ & $\begin{array}{l}114.705 w . \\
114.757 w . \\
114.660 w . \\
114.701 w . \\
114.665 w .\end{array}$ & $\begin{array}{l}005 \\
010 \\
020 \\
005 \\
019\end{array}$ & $\begin{array}{l}\mathrm{B} \\
\mathrm{B} \\
\mathrm{B} \\
\mathrm{B} \\
\mathrm{B}\end{array}$ & $\begin{array}{l}562 \\
562 \\
562 \\
562 \\
562\end{array}$ & $\begin{array}{l}\cdots \\
\cdots \\
\cdots \\
\cdots\end{array}$ & $\begin{array}{l}\cdots \\
\cdots \\
\cdots \\
\cdots\end{array}$ & $\begin{array}{l}\text { 3. } 5 \mathrm{ML} \\
\text { 3. } 3 \mathrm{ML} \\
\text { 3. } 4 \mathrm{ML} \\
\text { 4. } 0 \mathrm{ML} \\
\text { 3. } 3 \mathrm{ML}\end{array}$ & $\begin{array}{l}\text { GS } \\
\text { MMT } \\
\text { GS } \\
\text { GS } \\
\text { MMT }\end{array}$ & $\begin{array}{l}\cdots \\
\cdots \\
\cdots \\
\cdots\end{array}$ & $\begin{array}{l}\ldots \\
\cdots \\
\cdots \\
\cdots \\
\cdots\end{array}$ & $\begin{array}{l}\ldots \\
\cdots \\
\cdots \\
\cdots \\
\cdots\end{array}$ & $\begin{array}{l}\ldots \\
\cdots \\
\cdots \\
\cdots\end{array}$ \\
\hline $\begin{array}{l}1986 \\
1986 \\
1986 \\
1986 \\
1986\end{array}$ & $\begin{array}{l}\text { SEP } \\
\text { SEP } \\
\text { SEP } \\
\text { SEP } \\
\text { SEP }\end{array}$ & $\begin{array}{l}11 \\
14 \\
14 \\
16 \\
22\end{array}$ & $\begin{array}{l}08 \\
16 \\
21 \\
21 \\
05\end{array}$ & $\begin{array}{l}35 \\
01 \\
52 \\
00 \\
38\end{array}$ & $\begin{array}{l}32.1 \\
49.4 \\
04.5 \\
21.0 \\
51.9\end{array}$ & $\begin{array}{l}43.968 \mathrm{~N} . \\
43.968 \mathrm{~N} \\
43.979 \mathrm{~N} . \\
43.978 \mathrm{~N} . \\
44.040 \mathrm{~N} .\end{array}$ & $\begin{array}{l}114.647 w . \\
114.751 w . \\
114.679 w . \\
114.672 W . \\
114.756 w .\end{array}$ & $\begin{array}{l}021 \\
005 \\
019 \\
020 \\
005\end{array}$ & $\begin{array}{l}\mathrm{B} \\
\mathrm{C} \\
\mathrm{B} \\
\mathrm{B} \\
\mathrm{B}\end{array}$ & $\begin{array}{l}562 \\
562 \\
562 \\
562 \\
562\end{array}$ & $\begin{array}{l}\cdots \\
\cdots \\
\cdots \\
\cdots\end{array}$ & $\begin{array}{l}\cdots \\
\cdots \\
\cdots \\
\cdots\end{array}$ & $\begin{array}{l}3.1 \mathrm{ML} \\
3.1 \mathrm{ML} \\
3.1 \mathrm{ML} \\
3.5 \mathrm{ML} \\
3.4 \mathrm{ML}\end{array}$ & $\begin{array}{l}\text { MMT } \\
\text { GS } \\
\text { MMT } \\
\text { GS } \\
\text { GS }\end{array}$ & $\begin{array}{l}\cdots \\
\cdots \\
\cdots \\
\cdots\end{array}$ & $\begin{array}{l}\cdots \\
\cdots \\
\cdots \\
\cdots\end{array}$ & $\begin{array}{l}\cdots \\
\cdots \\
\cdots \\
\cdots \\
\cdots\end{array}$ & $\begin{array}{l}\cdots \\
\cdots \\
\cdots \\
\cdots\end{array}$ \\
\hline $\begin{array}{l}1986 \\
1986 \\
1986 \\
1986 \\
1986\end{array}$ & $\begin{array}{l}\text { SEP } \\
\text { SEP } \\
\text { SEP } \\
\text { SEP } \\
\text { SEP }\end{array}$ & $\begin{array}{l}24 \\
24 \\
26 \\
26 \\
26\end{array}$ & $\begin{array}{l}15 \\
15 \\
21 \\
22 \\
22\end{array}$ & $\begin{array}{l}32 \\
50 \\
28 \\
09 \\
48\end{array}$ & $\begin{array}{l}26.7 \\
47.8 \\
08.5 \\
48.3 \\
57.9\end{array}$ & $\begin{array}{l}44.003 \mathrm{~N} . \\
43.992 \mathrm{~N} . \\
44.016 \mathrm{~N} \\
43.959 \mathrm{~N} . \\
44.043 \mathrm{~N}\end{array}$ & $\begin{array}{l}114.755 w . \\
114.677 w . \\
114.750 w . \\
114.780 w . \\
114.756 w .\end{array}$ & $\begin{array}{l}005 \\
020 \\
005 \\
005 \\
005\end{array}$ & $\begin{array}{l}\mathrm{B} \\
\mathrm{B} \\
\mathrm{B} \\
\mathrm{C} \\
\mathrm{B}\end{array}$ & $\begin{array}{l}562 \\
562 \\
562 \\
562 \\
562\end{array}$ & $\begin{array}{c}\cdots \\
\cdots \\
4.6\end{array}$ & $\begin{array}{l}\cdots \\
\cdots \\
\cdots \\
\cdots\end{array}$ & $\begin{array}{l}3.7 \mathrm{ML} \\
3.2 \mathrm{ML} \\
4.3 \mathrm{ML} \\
3.6 \mathrm{ML} \\
4.5 \mathrm{ML}\end{array}$ & $\begin{array}{l}\text { GS } \\
\text { MMT } \\
\text { GS } \\
\text { MMT } \\
\text { GS }\end{array}$ & $\begin{array}{l}\cdots \\
\cdots \\
\cdots \\
\cdots\end{array}$ & $\begin{array}{l}\cdots \\
\cdots \\
\cdots \\
\cdots \\
\cdots\end{array}$ & $\begin{array}{r}\text { F } \\
\ddot{\text { IV }} \\
\ddot{\text { IV }}\end{array}$ & $\begin{array}{r}562 \\
5 \dot{62} \\
5 \dot{62}\end{array}$ \\
\hline $\begin{array}{l}1986 \\
1986 \\
1986 \\
1986 \\
1986\end{array}$ & $\begin{array}{l}\text { SEP } \\
\text { SEP } \\
\text { SEP } \\
\text { SEP } \\
\text { SEP }\end{array}$ & $\begin{array}{l}26 \\
27 \\
27 \\
27 \\
30\end{array}$ & $\begin{array}{l}22 \\
13 \\
18 \\
18 \\
11\end{array}$ & $\begin{array}{l}56 \\
02 \\
56 \\
59 \\
33\end{array}$ & $\begin{array}{l}12.1 \\
01.7 \\
17.7 \\
12.5 \\
33.8\end{array}$ & $\begin{array}{l}43.975 \mathrm{~N} . \\
44.017 \mathrm{~N} . \\
44.055 \mathrm{~N} . \\
43.996 \mathrm{~N} . \\
44.380 \mathrm{~N} .\end{array}$ & $\begin{array}{l}114.668 w . \\
114.780 w . \\
114.781 w . \\
114.680 w . \\
114.222 W .\end{array}$ & $\begin{array}{l}020 \\
005 \\
005 \\
019 \\
012\end{array}$ & $\begin{array}{l}\mathrm{B} \\
\mathrm{C} \\
\mathrm{C} \\
\mathrm{B} \\
\mathrm{B}\end{array}$ & $\begin{array}{l}562 \\
562 \\
562 \\
562 \\
562\end{array}$ & $\begin{array}{l}\cdots \\
\cdots \\
\cdots \\
\cdots\end{array}$ & $\begin{array}{l}\cdots \\
\cdots \\
\cdots \\
\cdots\end{array}$ & $\begin{array}{l}3.2 \mathrm{ML} \\
3.3 \mathrm{ML} \\
3.3 \mathrm{ML} \\
3.8 \mathrm{ML} \\
3.6 \mathrm{ML}\end{array}$ & $\begin{array}{l}\text { MMT } \\
\text { GS } \\
\text { GS } \\
\text { GS } \\
\text { MMT }\end{array}$ & $\begin{array}{l}\cdots \\
\cdots \\
\cdots \\
\cdots\end{array}$ & $\begin{array}{l}\cdots \\
\cdots \\
\ldots \\
\ldots \\
\ldots\end{array}$ & $\begin{array}{l}\cdots \\
\ldots \\
\ldots \\
\ldots \\
\ldots\end{array}$ & $\begin{array}{l}\cdots \\
\cdots \\
\cdots \\
\cdots\end{array}$ \\
\hline $\begin{array}{l}1986 \\
1986 \\
1986 \\
1986 \\
1986\end{array}$ & $\begin{array}{l}\text { SEP } \\
\text { OCT } \\
\text { OCT } \\
\text { OCT } \\
\text { OCT }\end{array}$ & $\begin{array}{l}30 \\
01 \\
02 \\
03 \\
09\end{array}$ & $\begin{array}{l}16 \\
07 \\
09 \\
18 \\
09\end{array}$ & $\begin{array}{l}11 \\
20 \\
02 \\
58 \\
42\end{array}$ & $\begin{array}{l}53.5 \\
29.6 \\
24.6 \\
35.2 \\
41.5\end{array}$ & $\begin{array}{l}43.996 \mathrm{~N} . \\
43.978 \mathrm{~N} . \\
44.405 \mathrm{~N} . \\
44.358 \mathrm{~N} . \\
43.961 \mathrm{~N} .\end{array}$ & $\begin{array}{l}113.942 W . \\
114.780 W . \\
114.054 W . \\
112.596 w . \\
114.756 w .\end{array}$ & $\begin{array}{l}005 \\
005 \\
022 \\
016 \\
005\end{array}$ & $\begin{array}{l}B \\
C \\
C \\
B \\
C\end{array}$ & $\begin{array}{l}562 \\
562 \\
562 \\
562 \\
562\end{array}$ & $\begin{array}{l}\cdots \\
\cdots \\
\cdots \\
\cdots\end{array}$ & $\begin{array}{l}\cdots \\
\cdots \\
\cdots \\
\cdots\end{array}$ & $\begin{array}{l}3.6 \mathrm{ML} \\
3.0 \mathrm{ML} \\
3.4 \mathrm{ML} \\
2.5 \mathrm{ML} \\
3.1 \mathrm{ML}\end{array}$ & $\begin{array}{l}\text { GS } \\
\text { GS } \\
\text { MMT } \\
\text { MMT } \\
\text { GS }\end{array}$ & $\begin{array}{l}\cdots \\
\cdots \\
\cdots \\
\cdots\end{array}$ & $\begin{array}{l}\cdots \\
\cdots \\
\cdots \\
\cdots \\
\cdots\end{array}$ & $\begin{array}{l}\cdots \\
\ldots \\
\cdots \\
\ldots \\
\cdots\end{array}$ & $\begin{array}{l}\cdots \\
\cdots \\
\cdots \\
\cdots\end{array}$ \\
\hline $\begin{array}{l}1986 \\
1986 \\
1986 \\
1986 \\
1986\end{array}$ & $\begin{array}{l}\text { OCT } \\
\text { OCT } \\
\text { OCT } \\
\text { OCT } \\
\text { OCT }\end{array}$ & $\begin{array}{l}10 \\
14 \\
14 \\
14 \\
18\end{array}$ & $\begin{array}{l}23 \\
12 \\
13 \\
13 \\
21\end{array}$ & $\begin{array}{l}45 \\
17 \\
10 \\
43 \\
21\end{array}$ & $\begin{array}{l}04.9 \\
53.3 \\
09.8 \\
47.5 \\
28.7\end{array}$ & $\begin{array}{l}43.976 \mathrm{~N} \\
44.023 \mathrm{~N} . \\
44.058 \mathrm{~N} \\
44.066 \mathrm{~N} \\
42.064 \mathrm{~N}\end{array}$ & $\begin{array}{l}114.675 w . \\
114.674 w . \\
114.712 W . \\
114.684 w . \\
111.454 W .\end{array}$ & $\begin{array}{l}020 \\
005 \\
005 \\
005 \\
005\end{array}$ & $\begin{array}{l}\text { B } \\
B \\
B \\
B \\
B\end{array}$ & $\begin{array}{l}562 \\
562 \\
562 \\
562 \\
562\end{array}$ & $\begin{array}{l}\cdots \\
\cdots \\
\cdots \\
\cdots\end{array}$ & $\begin{array}{l}\cdots \\
\cdots \\
\cdots \\
\cdots\end{array}$ & $\begin{array}{l}2.9 \mathrm{ML} \\
3.9 \mathrm{ML} \\
3.9 \mathrm{ML} \\
3.6 \mathrm{ML} \\
3.5 \mathrm{ML}\end{array}$ & $\begin{array}{l}\text { MMT } \\
\text { GS } \\
\text { GS } \\
\text { GS } \\
\text { GS }\end{array}$ & $\begin{array}{l}\cdots \\
\cdots \\
\cdots \\
\cdots\end{array}$ & $\begin{array}{l}\cdots \\
\cdots \\
\cdots \\
\cdots \\
\cdots\end{array}$ & $\begin{array}{l}\ddot{\text { IV }} \\
\cdots \\
\ddot{\text { IV }}\end{array}$ & $\begin{array}{r}5 \dot{62} \\
\ldots \\
5 \dot{62}\end{array}$ \\
\hline $\begin{array}{l}1986 \\
1986 \\
1986 \\
1986 \\
1986\end{array}$ & $\begin{array}{l}\text { OCT } \\
\text { NOV } \\
\text { NOV } \\
\text { NOV } \\
\text { NOV }\end{array}$ & $\begin{array}{l}25 \\
01 \\
03 \\
04 \\
07\end{array}$ & $\begin{array}{l}20 \\
01 \\
17 \\
08 \\
12\end{array}$ & $\begin{array}{l}27 \\
39 \\
08 \\
46 \\
44\end{array}$ & $\begin{array}{l}25.4 \\
12.6 \\
19.5 \\
01.7 \\
14.2\end{array}$ & $\begin{array}{l}43.985 \mathrm{~N} . \\
44.399 \mathrm{~N} . \\
44.010 \mathrm{~N} . \\
44.187 \mathrm{~N} . \\
44.073 \mathrm{~N} .\end{array}$ & $\begin{array}{l}114.673 w . \\
114.049 w . \\
114.579 w . \\
114.065 w . \\
114.482 W .\end{array}$ & $\begin{array}{l}020 \\
027 \\
015 \\
005 \\
005\end{array}$ & $\begin{array}{l}B \\
C \\
C \\
B \\
C\end{array}$ & $\begin{array}{l}562 \\
562 \\
562 \\
562 \\
562\end{array}$ & $\begin{array}{l}\cdots \\
\cdots \\
\cdots \\
\cdots\end{array}$ & $\begin{array}{l}\cdots \\
\cdots \\
\cdots \\
\cdots\end{array}$ & $\begin{array}{l}2.9 \mathrm{ML} \\
2.9 \mathrm{ML} \\
3.4 \mathrm{ML} \\
3.5 \mathrm{ML} \\
3.0 \mathrm{ML}\end{array}$ & $\begin{array}{l}\text { MMT } \\
\text { MMT } \\
\text { MMT } \\
\text { GS } \\
\text { GS }\end{array}$ & $\begin{array}{l}\cdots \\
\cdots \\
\cdots \\
\cdots\end{array}$ & $\begin{array}{l}\cdots \\
\cdots \\
\cdots \\
\cdots \\
\cdots\end{array}$ & $\begin{array}{l}\cdots \\
\cdots \\
\cdots \\
\cdots\end{array}$ & $\begin{array}{l}\cdots \\
\cdots \\
\cdots \\
\cdots\end{array}$ \\
\hline $\begin{array}{l}1986 \\
1986 \\
1986 \\
1986 \\
1986\end{array}$ & $\begin{array}{l}\text { NOV } \\
\text { NOV } \\
\text { NOV } \\
\text { NOV } \\
\text { NOV }\end{array}$ & $\begin{array}{l}09 \\
09 \\
09 \\
12 \\
15\end{array}$ & $\begin{array}{l}14 \\
14 \\
17 \\
07 \\
09\end{array}$ & $\begin{array}{l}02 \\
15 \\
16 \\
12 \\
00\end{array}$ & $\begin{array}{l}26.4 \\
37.8 \\
42.0 \\
47.5 \\
13.2\end{array}$ & $\begin{array}{l}43.979 \mathrm{~N} . \\
44.024 \mathrm{~N} . \\
43.979 \mathrm{~N} \\
44.004 \mathrm{~N} \\
42.706 \mathrm{~N}\end{array}$ & $\begin{array}{l}114.740 w . \\
114.723 w . \\
114.640 w . \\
114.715 w . \\
111.667 w .\end{array}$ & $\begin{array}{l}005 \\
005 \\
017 \\
005 \\
005\end{array}$ & $\begin{array}{l}C \\
C \\
C \\
C \\
B\end{array}$ & $\begin{array}{l}562 \\
562 \\
562 \\
562 \\
562\end{array}$ & $\begin{array}{l}\cdots \\
\cdots \\
\cdots \\
\cdots\end{array}$ & $\begin{array}{l}\cdots \\
\cdots \\
\cdots \\
\cdots\end{array}$ & $\begin{array}{l}3.8 \mathrm{ML} \\
3.3 \mathrm{ML} \\
2.9 \mathrm{ML} \\
3.0 \mathrm{ML} \\
3.3 \mathrm{ML}\end{array}$ & $\begin{array}{l}\text { GS } \\
\text { GS } \\
\text { MMT } \\
\text { GS } \\
\text { GS }\end{array}$ & $\begin{array}{l}\cdots \\
\cdots \\
\cdots \\
\cdots\end{array}$ & $\begin{array}{l}\cdots \\
\cdots \\
\cdots \\
\cdots \\
\cdots\end{array}$ & $\begin{array}{l}\cdots \\
\cdots \\
\cdots \\
\text { IV }\end{array}$ & $\begin{array}{l}\cdots \\
\cdots \\
5 \dot{6} \dot{2}\end{array}$ \\
\hline $\begin{array}{l}1986 \\
1986 \\
1986 \\
1986 \\
1986\end{array}$ & $\begin{array}{l}\text { NOV } \\
\text { NOV } \\
\text { NOV } \\
\text { NOV } \\
\text { DEC }\end{array}$ & $\begin{array}{l}18 \\
21 \\
23 \\
26 \\
02\end{array}$ & $\begin{array}{l}16 \\
08 \\
06 \\
00 \\
15\end{array}$ & $\begin{array}{l}09 \\
07 \\
03 \\
13 \\
24\end{array}$ & $\begin{array}{l}40.3 \\
55.0 \\
09.9 \\
10.1 \\
56.9\end{array}$ & $\begin{array}{l}43.976 \mathrm{~N} \\
44.523 \mathrm{~N} \\
44.758 \mathrm{~N} \\
42.486 \mathrm{~N} \\
43.982 \mathrm{~N}\end{array}$ & $\begin{array}{l}114.754 w . \\
114.025 w . \\
114.387 w . \\
111.260 w . \\
114.756 w .\end{array}$ & $\begin{array}{l}018 \\
006 \\
016 \\
001 \\
016\end{array}$ & $\begin{array}{l}C \\
C \\
C \\
B \\
B\end{array}$ & $\begin{array}{l}562 \\
562 \\
562 \\
562 \\
562\end{array}$ & $\begin{array}{l}\cdots \\
\cdots \\
\cdots \\
\cdots\end{array}$ & $\begin{array}{l}\ldots \\
\ldots \\
\ldots \\
\ldots \\
\ldots\end{array}$ & $\begin{array}{l}3.6 \mathrm{MD} \\
2.6 \mathrm{ML} \\
2.5 \mathrm{ML} \\
3.5 \mathrm{ML} \\
2.8 \mathrm{ML}\end{array}$ & $\begin{array}{l}\text { MMT } \\
\text { MMT } \\
\text { MMT } \\
\text { UU } \\
\text { MMT }\end{array}$ & $\begin{array}{l}\cdots \\
\cdots \\
\cdots \\
\cdots\end{array}$ & $\begin{array}{l}\cdots \\
\cdots \\
\cdots \\
\cdots \\
\cdots\end{array}$ & $\begin{array}{l}\cdots \\
\cdots \\
\cdots \\
\cdots \\
\cdots\end{array}$ & $\begin{array}{l}\cdots \\
\cdots \\
\cdots \\
\cdots\end{array}$ \\
\hline $\begin{array}{l}1986 \\
1986 \\
1986 \\
1986 \\
1986\end{array}$ & $\begin{array}{l}\text { DEC } \\
\text { DEC } \\
\text { DEC } \\
\text { DEC } \\
\text { DEC }\end{array}$ & $\begin{array}{l}02 \\
11 \\
13 \\
15 \\
20\end{array}$ & $\begin{array}{l}23 \\
12 \\
18 \\
20 \\
07\end{array}$ & $\begin{array}{l}58 \\
35 \\
12 \\
50 \\
18\end{array}$ & $\begin{array}{l}09.8 \\
53.1 \\
06.3 \\
27.6 \\
47.5\end{array}$ & $\begin{array}{l}43.965 \mathrm{~N} \\
44.638 \mathrm{~N} \\
44.213 \mathrm{~N} \\
44.377 \mathrm{~N} \\
44.335 \mathrm{~N}\end{array}$ & $\begin{array}{l}114.627 W . \\
114.093 W . \\
114.010 W . \\
114.152 W . \\
114.454 W .\end{array}$ & $\begin{array}{l}026 \\
010 \\
005 \\
005 \\
005\end{array}$ & $\begin{array}{l}C \\
C \\
C \\
B \\
C\end{array}$ & $\begin{array}{l}562 \\
562 \\
562 \\
562 \\
562\end{array}$ & $\begin{array}{l}\cdots \\
\cdots \\
\cdots \\
\cdots\end{array}$ & $\begin{array}{l}\ldots \\
\ldots \\
\ldots \\
\ldots \\
\cdots\end{array}$ & $\begin{array}{l}3.0 \mathrm{ML} \\
2.6 \mathrm{ML} \\
3.2 \mathrm{ML} \\
2.8 \mathrm{ML} \\
3.0 \mathrm{ML}\end{array}$ & $\begin{array}{l}\text { MMT } \\
\text { MMT } \\
\text { GS } \\
\text { GS } \\
\text { GS }\end{array}$ & $\begin{array}{l}\cdots \\
\cdots \\
\cdots \\
\cdots\end{array}$ & $\begin{array}{l}\ldots \\
\cdots \\
\cdots \\
\cdots \\
\ldots\end{array}$ & $\begin{array}{l}\cdots \\
\cdots \\
\cdots \\
\cdots \\
\cdots\end{array}$ & $\begin{array}{l}\cdots \\
\cdots \\
\cdots \\
\cdots \\
\cdots\end{array}$ \\
\hline $\begin{array}{l}1986 \\
1986 \\
1987\end{array}$ & $\begin{array}{l}\text { DEC } \\
\text { DEC } \\
\text { JAN }\end{array}$ & $\begin{array}{l}24 \\
30 \\
12\end{array}$ & $\begin{array}{l}07 \\
09 \\
03\end{array}$ & $\begin{array}{l}28 \\
51 \\
05\end{array}$ & $\begin{array}{l}58.4 \\
28.3 \\
12.9\end{array}$ & $\begin{array}{l}43.878 \mathrm{~N} \\
42.720 \mathrm{~N} \\
42.706 \mathrm{~N}\end{array}$ & $\begin{array}{l}114.848 W . \\
111.275 w . \\
111.072 W .\end{array}$ & $\begin{array}{l}005 \\
005 \\
005\end{array}$ & $\begin{array}{l}C \\
B \\
B\end{array}$ & $\begin{array}{l}562 \\
562 \\
577\end{array}$ & $\begin{array}{l}\cdots \\
\cdots \\
\cdots\end{array}$ & $\cdots$ & $\begin{array}{l}3.0 \mathrm{ML} \\
3.2 \mathrm{ML} \\
3.7 \mathrm{ML}\end{array}$ & $\begin{array}{l}\text { GS } \\
\text { GS } \\
\text { GS }\end{array}$ & $\begin{array}{l}\cdots \\
\cdots \\
\cdots\end{array}$ & $\begin{array}{l}\cdots \\
\cdots \\
\cdots\end{array}$ & I I I & $\begin{array}{c}\ldots \\
577\end{array}$ \\
\hline
\end{tabular}




\begin{tabular}{|c|c|c|c|c|c|c|c|c|c|c|c|c|c|c|c|c|c|c|}
\hline $\begin{array}{l}1987 \\
1987\end{array}$ & $\begin{array}{l}\text { JAN } \\
\text { JAN }\end{array}$ & $\begin{array}{l}14 \\
14\end{array}$ & $\begin{array}{l}00 \\
06\end{array}$ & $\begin{array}{l}22 \\
52\end{array}$ & $\begin{array}{l}22.4 \\
09.9\end{array}$ & $\begin{array}{l}44.597 N \\
44.636 \mathrm{~N}\end{array}$ & $\begin{array}{l}114.864 w . \\
114.875 w .\end{array}$ & $\begin{array}{l}005 \\
005\end{array}$ & $\begin{array}{l}\text { B } \\
\text { B }\end{array}$ & $\begin{array}{l}577 \\
577\end{array}$ & $\begin{array}{l}\cdots \\
\cdots\end{array}$ & 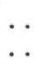 & $\begin{array}{l}\text { 3. } 1 M L \\
3.5 M L\end{array}$ & $\begin{array}{l}\text { GS } \\
\text { GS }\end{array}$ & 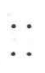 & $\cdots$ & III & $5 \ddot{77}$ \\
\hline $\begin{array}{l}1987 \\
1987 \\
1987 \\
1987 \\
1987\end{array}$ & $\begin{array}{l}\text { JAN } \\
\text { JAN } \\
\text { MAR } \\
\text { MAR } \\
\text { APR }\end{array}$ & $\begin{array}{l}19 \\
20 \\
18 \\
23 \\
26\end{array}$ & $\begin{array}{l}13 \\
08 \\
00 \\
14 \\
14\end{array}$ & $\begin{array}{l}24 \\
34 \\
00 \\
04 \\
41\end{array}$ & $\begin{array}{l}19.9 \\
04.6 \\
42.9 \\
35.8 \\
42.2\end{array}$ & $\begin{array}{l}44.027 \mathrm{~N} \\
44.316 \mathrm{~N} \\
42.581 \mathrm{~N} \\
44.439 \mathrm{~N} \\
44.107 \mathrm{~N}\end{array}$ & $\begin{array}{l}114.860 w . \\
114.290 w . \\
111.299 w . \\
114.088 w . \\
114.612 W .\end{array}$ & $\begin{array}{l}005 \\
005 \\
005 \\
005 \\
005\end{array}$ & $\begin{array}{l}C \\
B \\
B \\
B \\
B\end{array}$ & $\begin{array}{l}577 \\
577 \\
577 \\
577 \\
577\end{array}$ & $\begin{array}{r}\ldots \\
3.9 \\
\ldots\end{array}$ & $\begin{array}{l}\cdots \\
\cdots \\
\cdots \\
\cdots\end{array}$ & $\begin{array}{l}\text { 3. } 6 \mathrm{ML} \\
\text { 3. } \mathrm{OML} \\
\text { 4. } 3 \mathrm{ML} \\
\text { 3. } 3 \mathrm{ML} \\
\text { 3. } 5 \mathrm{ML}\end{array}$ & $\begin{array}{l}\text { GS } \\
\text { GS } \\
\text { GS } \\
\text { GS } \\
\text { GS }\end{array}$ & $\begin{array}{l}\cdots \\
\cdots \\
\cdots \\
\cdots\end{array}$ & $\begin{array}{l}\cdots \\
\cdots \\
\cdots \\
\cdots\end{array}$ & $\begin{array}{l}\cdots \\
\text { IV } \\
\text { III }\end{array}$ & $\begin{array}{r}\ldots \\
577 \\
577\end{array}$ \\
\hline $\begin{array}{l}1987 \\
1987 \\
1987 \\
1987 \\
1987\end{array}$ & $\begin{array}{l}\text { MAY } \\
\text { JUL } \\
\text { JUL } \\
\text { AUG } \\
\text { AUG }\end{array}$ & $\begin{array}{l}26 \\
24 \\
25 \\
04 \\
17\end{array}$ & $\begin{array}{l}16 \\
23 \\
16 \\
06 \\
03\end{array}$ & $\begin{array}{l}11 \\
54 \\
54 \\
37 \\
44\end{array}$ & $\begin{array}{l}47.1 \\
05.9 \\
40.1 \\
48.9 \\
19.9\end{array}$ & $\begin{array}{l}45.400 \mathrm{~N} \\
44.172 \mathrm{~N} \\
42.123 \mathrm{~N} \\
44.264 \mathrm{~N} \\
44.046 \mathrm{~N}\end{array}$ & $\begin{array}{l}116.261 W . \\
114.631 w . \\
112.472 W . \\
114.315 W . \\
114.747 W .\end{array}$ & $\begin{array}{l}005 \\
005 \\
004 \\
005 \\
005\end{array}$ & $\begin{array}{l}\mathrm{B} \\
\mathrm{C} \\
\mathrm{B} \\
\mathrm{C} \\
\mathrm{B}\end{array}$ & $\begin{array}{l}577 \\
577 \\
577 \\
577 \\
577\end{array}$ & $\begin{array}{l}\ldots \\
\ldots \\
\cdots \\
4.0\end{array}$ & $\begin{array}{l}\cdots \\
\cdots \\
\cdots \\
\cdots\end{array}$ & $\begin{array}{l}\text { 3. } 6 \mathrm{ML} \\
\text { 3. } 2 \mathrm{ML} \\
\text { 2. } 8 \mathrm{ML} \\
\text { 3. } 6 \mathrm{ML} \\
\text { 4. } 4 \mathrm{ML}\end{array}$ & $\begin{array}{l}\text { GS } \\
\text { MMT } \\
\text { UU } \\
\text { MMT } \\
\text { GS }\end{array}$ & $\begin{array}{l}\cdots \\
\cdots \\
\cdots \\
\cdots\end{array}$ & $\begin{array}{l}\cdots \\
\cdots \\
\cdots \\
\cdots \\
\cdots\end{array}$ & $\begin{array}{l}\quad v \\
\cdots \\
\cdots \\
i \dot{I I}\end{array}$ & $\begin{array}{r}577 \\
\ldots \\
\ldots \\
5 \ddot{7}\end{array}$ \\
\hline $\begin{array}{l}1987 \\
1987 \\
1987 \\
1987 \\
1987\end{array}$ & $\begin{array}{l}\text { SEP } \\
\text { SEP } \\
\text { OCT } \\
\text { OCT } \\
\text { NOV }\end{array}$ & $\begin{array}{l}10 \\
12 \\
12 \\
18 \\
04\end{array}$ & $\begin{array}{l}04 \\
11 \\
22 \\
06 \\
23\end{array}$ & $\begin{array}{l}45 \\
36 \\
46 \\
36 \\
17\end{array}$ & $\begin{array}{l}56.8 \\
55.3 \\
14.2 \\
07.6 \\
52.0\end{array}$ & $\begin{array}{l}44.686 \mathrm{~N} \\
44.499 \mathrm{~N} \\
44.311 \mathrm{~N} \\
44.622 \mathrm{~N} \\
43.231 \mathrm{~N}\end{array}$ & $\begin{array}{l}114.195 W . \\
114.522 W . \\
114.015 W . \\
114.401 W . \\
111.193 W .\end{array}$ & $\begin{array}{l}005 \\
005 \\
005 \\
005 \\
005\end{array}$ & $\begin{array}{l}B \\
D \\
B \\
B \\
C\end{array}$ & $\begin{array}{l}577 \\
577 \\
577 \\
577 \\
577\end{array}$ & $\begin{array}{l}\cdots \\
\cdots \\
\cdots \\
\cdots\end{array}$ & $\begin{array}{l}\cdots \\
\cdots \\
\cdots \\
\cdots\end{array}$ & $\begin{array}{l}\text { 3. } 4 \mathrm{ML} \\
2.9 \mathrm{ML} \\
3.7 \mathrm{ML} \\
3.7 \mathrm{ML} \\
2.5 \mathrm{ML}\end{array}$ & $\begin{array}{l}\text { GS } \\
\text { GS } \\
\text { GS } \\
\text { GS } \\
\text { GS }\end{array}$ & $\begin{array}{l}\cdots \\
\cdots \\
\cdots \\
\cdots\end{array}$ & $\begin{array}{l}\ldots \\
\ldots \\
\ldots \\
\ldots \\
\ldots\end{array}$ & $\begin{array}{r}\text { F } \\
\text { III } \\
\ldots \\
\ldots\end{array}$ & $\begin{array}{r}577 \\
577 \\
\ldots \\
\ldots\end{array}$ \\
\hline
\end{tabular}


2. Heck, N. H. and Bodle, R. R., 1931, United States earthquakes 1929: U. S. Coost and Geodetic Survey, Serial $511,55 \mathrm{p}$.

4. Neumann, Frank, 1932, United States earthquakes 1931: U. S. Coast and Geodetic Survey, Serial 553, 26 p.

5. Neumann. Frank, 1934, United States earthquakes 1932: Survey. Serial 563, $21 \mathrm{p}$.

6. Neumann. Frank, 1935, United States earthquakes 1933: Survey, Serial 579, 82 p.

7. Neumann. Frank, 1936, United States eorthquakes 1934: Survey. Serial 593, 99 p.

8. Neumann. Frank, 1937, United States earthquakes 1935: Survey, Serial 600,90 p.

9. Neumann, Frank, 1938, United States carthquakes 1936: Survey, Serial 610, 45 p.

11. Neumann, Frank, 1940, United States earthquakes 1938: Survey, Serial 629, 59 p.

13. Neumann, Frank, 1942, United States earthquakes 1940: Survey. Serial 647, $74 \mathrm{p}$.

15. Bodle, R. R.. 1944, United States earthquakes 1942: Survey, Serial 662, $44 \mathrm{p}$.

16. Bodle, R. R.. 1945, United Stotes earthquakes 1943: Survey. Serial 672, $47 \mathrm{p}$.

17. Bodle, R. R., 1946, United States earthquakes 1944: Survey, Serial 682, 43 p.

18. Bodle, R. R., and Murphy, $i$. M., 1947, United States earthquakes 1945: U. S. Coost and Geodetic Survey, Serial 699,38 p.

20. Murphy, L. M., 1950, United States earthquakes 1947: U. S. Coast and Geodetic Survey, Serial 730, 62 p.

22. Murphy, L. M., and Uirich, F. P., 1951, United States earthquakes 1949: U. S. Coast and Geodetic Survey, Serial 748, $64 \mathrm{p}$.

23. Murphy, L. M. , and UIrich, F. P., 1952, United States earthquakes 1950: U. S. Coast and Geodetic Survey, Seriai 755, 47 p.

25. Murphy, L. M. , and Cloud, W. K., 1954, United States earthquakes 1952: U. S. Coost and Geodetic Survey, Serial 773, 112 p.

26. Murphy, L. M., and Cloud, W. K., 1955, United States earthquakes 1953: U. S. Coast and Geodetic Survey, Serial 785,51 .

27. Murphy, L. M., and Cloud, W. K., 1956, United States earthquakes 1954: U. S. Coost and Geodetic Survey, Serial $793,110 \mathrm{p}$.

28. Murphy, L. M., and Cloud, W. K., 1957, United States earthquakes 1955: U. S. Coast and Geodetic Survey, $83 \mathrm{p}$.

29. Brazee, R. J., and Cloud, W. K., 1958, United States earthquakes 1956: U. S. Coost and Geodetic Survey, $78 \mathrm{p}$.

30. Brazee, R. J., and Cloud, W. K., 1959, United States earthquakes 1957: U. S. Coast and Geodetic Survey, $108 \mathrm{p}$.

31. Brazee, R. J., and Cloud, W. K., 1960, United States earthquakes 1958: U. S. Coast and Geodetic Survey, $76 \mathrm{p}$.

32. Eppley, R. A., and Cloud, W. K., 1961, United States earthquakes 1959: U. S. Coast and Geodetic Survey, $115 \mathrm{p}$.

33. Talley, H. C., and Cloud, W. K., 1962, United States earthquakes 1960: U. S. Coast and Geodetic Survey, $90 \mathrm{p}$.

34. Lander, J. F., and Cloud, W. K., 1963, United States earthquakes 1961: U. S. Coast and Geodetic Survey, $106 \mathrm{p}$.

35. Lander, J. F., and Cloud, W. K., 1964, United States earthquakes 1962: U. S. Coast and Geodetic Survey, 114 p.

36. Cloud, W. K., and von Hake, C. A., 1965, United States earthquakes 1963: U. S. Coost and Geodetic Survey, $69 \mathrm{p}$.

37. von Hake, C. A., and Cloud, W. K., 1966, United States earthquakes 1964: U. S. Coost and Geodetic Survey, $91 \mathrm{p}$.

38. Coffman, J. L., von Hake, C. A., and Stover, C. W., 1982, Earthquake history of the United States: $U . S$. Notional Oceonic and Atmospheric Administration and $U . S$. Geological Survey. Publication No. 41-1(through 1980), 258 p.

39. Coffman, J. L., and Stover, C. W., 1979, United States earthquakes 1977: U. S. Notional Oceanic and Atmospheric Administration and U. S. Geological Survey, 81 .

41. Coffman, J. L., and Cloud, W. K., 1970, United States earthquakes 1968: U. S. Environmentai Science Services Administration, $111 \mathrm{p}$.

42. von Hake, C. A., and Cloud, W. K., 1971, United States earthquakes 1969: U. S. National Oceanic and Atmospheric Administration, $80 \mathrm{p}$.

44. Coffman, J. L., and von Hake, C. A., 1973, United States earthquakes 1971: U. S. National Oceanic and Atmospheric Administration, $174 \mathrm{p}$.

45. Coffman, J. L., and von Hake, C. A., 1974, United States earthquakes 1972: U. S. National Oceanic and Atmospheric Administration, $119 \mathrm{p}$. 
46. Coffman, J. L., von Hake, C. A., Spence, W., Carver, D. L., Covington, P. A., Dunphy, G. J.. Irby, W. L., Person, W. J., and Stover, C. W. , 1975, United States earthquakes 1973: U. S. Notional Oceanic and Atmospheric Administration and U. S. Geological Survey, 112 p.

47. Coffman, J. L., and Stover, C. W., 1976, United States earthquakes 1974: U. S. National Oceonic and Atmospheric Administration and U.S. Geological Survey, 135 p.

48. Coffman, J. L., and Stover, C. W., 1977, United States earthquakes 1975: U. S. National Oceonic and Atmospheric Administration and U.S. Geological Survey, $136 \mathrm{p}$.

49. Coffman, J. L., and Stover, C. W. 1978, United States earthquakes 1976: U. S. National Oceanic and Atmospheric Administration and U. S. Geological Survey, $94 p$.

52. Williams, J. S., and Tapper, M. S., 1953, Earthquake history of Utah, 1850 to 1949: Seismological Society of America Bulletin, v. 43, no. 3, p. 191-218.

54. Slemmons, D. B., Jones, A. E., and Gimlett, J. I., 1965, Catalog of Nevodo earthquakes, 1852-1960: Seismological Society of America Bulletin, v. 55, no. 2, p. 519-565.

56. Townley, S. D., and Allen, M. W., 1939, Descriptive catalog of earthquakes of the Pacific coast of the United States, 1769 to 1928: Seismological Society of America Bulletin, v. 29 , no. 1, p. 1-297.

72. U. S. Coast and Geodetic Survey. Abstracts of earthquake reports for the United States, 1967 through 1973.

74. U. S. Geological Survey. Preliminary determination of epicenters Report and Monthly Listing, Januory 1961-present, formerly by U. S. Coast and Geodetic Survey, U. S. Environmental Science Services Administration, and $U$. S. National Oceanic and Atmospheric Administration.

75. von Hake, C. A., and Cloud, W. K., 1967, United States Earthquakes 1965: U. S. Coost and Geodetic Survey, $91 \mathrm{p}$.

81. von Hake, C. A., and Cloud, W. K., 1968, United States earthquakes 1966: U. S. Coast and Geodetic Survey, 110 p.

218. U. S. Coast and Geodetic Survey, Seismological Reports, July 1924 through December 1927.

234. Cook, K. L., and Smith, R. B., 1967, Seismicity in Utah, 1850 through June 1965: Seismological Society of America Bulletin, v. 57, no. 4, p. 689-718.

240. Stover, C. W., and von Hake, C. A., 1980, United States earthquakes 1978: U. S. Geological Survey and U.S. National Oceanic and Atmospheric Administration, $112 p$.

249. Qamar, A. I., and Stickney, M. C., 1983, Montana earthquakes 1869-1979: Montano Bureau of Mines and Geology Memoir 51, 79 p.

259. U. S. Coast and Geodetic Survey. Abstrocts of earthquake reports for the Pacific Coast and the Western Mountain Region, January 1, 1934 to December 31, 1966, and 1933 abstracts from the San Francisco of fice, U. S. Department of Commerce.

260. Stover, C.W., 1990, U. S. Geological Survey, re-computed hypocenters (unpublished data).

262. Stover, C. W., and von Hake, C. A., 1981, United States earthquakes 1979: U. S. Geological Survey and U.S. Notional Oceanic and Atmospheric Administration, $170 \mathrm{p}$

266. U. S. Coast and Geodetic Survey, Seismological Bulletin MSI 1 through MSI 316, January 1934 through May 1967.

272. Monthly Weother Review, July 1891 to June 1924, v. 19 to 68, U. S. Department of Agriculture, Woshington, D. C.

293. Seismological Society of America, 1937, Seismological Notes, Seismological Society of America Bulletin, v. 27, no. 3, P. 255-257.

298. Arabasz, W. J., Smith, R. B., and Richins, W. D., 1979, Earthquake studies in Utah 1850 to 1978: University of Utah, Department of Geology and Geophysics, 552 p.

299. Bulletin of the International Seismological Centre, 1964 to present, Newbury, Berkshire, United Kingdom.

300. Stover, C. $\dot{W}$. , and von Hake, C. A., 1982, United States earthquakes 1980: U. S. Geological Survey and U.S. National Oceanic and Atmospheric Administration, 182 p.

325. Stover, C. W., 1984, United States earthquakes 1981: U. S. Geological Survey Special Publicotion, $136 \mathrm{p}$.

334. Lander, J. F., 1969, Seismological Notes: Seismological Society of America Bulletin, v.59, no. 2, p. 445-458.

350. Stover, C. W. 1985, United States earthquakes, 1982: U. S. Geological Survey Bulletin 1655, $142 \mathrm{p}$.

352. Union Geodesique et Geophysique Internationale, Bureau Central International de Seismologie. Bulletin Mensuel, 1953 through 1972.

354. Dewey, J. W. 1987, Instrumentai seismicity of central Idaho: Seismological Society of Americo Bulletin, v. 77 , no. 3, p. 819-836.

360. Stover, C. W.. 1986, United States eorthquakes, 1983: U. S. Geological Survey Bulletin 1698, $197 \mathrm{p}$.

370. Stover, C. W., 1988, United States Earthquakes, 1984: U. S. Geological Survey Bulletin 1862, $284 \mathrm{p}$.

371. Stover, C. W., and Brewer, L. R., 1991, United States Earthquakes, 1985: U. S. Geological Survey Bullet in 1954, 170 p.

562. Stover, C. W.. and Brewer, L. B., 1991, United Stotes Earthquakes, 1986: U. S. Geological Survey Bulletin (in preparation).

577. Stover, C. W. and Brower, L. B., 1992, United States Earthquakes, 1987: U. S. Geological Survey Bulletin (in preparation). 